\title{
EVOLUTION AND NUCLEOSYNTHESIS OF ASYMPTOTIC GIANT BRANCH STELLAR MODELS OF LOW METALLICITY
}

\author{
Cherie K. Fishlock ${ }^{1}$, Amanda I. KaraKas ${ }^{1}$, Maria Lugaro ${ }^{2}$, and David Yong ${ }^{1}$ \\ ${ }^{1}$ Research School of Astronomy and Astrophysics, Australian National University, Canberra ACT 2611, \\ Australia, cherie.fishlock@anu.edu.au, amanda.karakas@anu.edu.au,david.yong@anu.edu.au \\ 2 Monash Centre for Astrophysics, Monash University, Clayton VIC 3800, Australia; maria.lugaro@monash.edu \\ Received 2014 May 23; accepted 2014 October 17; published 2014 November 24
}

\begin{abstract}
We present stellar evolutionary tracks and nucleosynthetic predictions for a grid of stellar models of low- and intermediate-mass asymptotic giant branch (AGB) stars at $Z=0.001([\mathrm{Fe} / \mathrm{H}]=-1.2)$. The models cover an initial mass range from $1 M_{\odot}$ to $7 M_{\odot}$. Final surface abundances and stellar yields are calculated for all elements from hydrogen to bismuth as well as isotopes up to the iron group. We present the first study of neutron-capture nucleosynthesis in intermediate-mass AGB models, including a super-AGB model, of $[\mathrm{Fe} / \mathrm{H}]=-1.2$. We examine in detail a low-mass AGB model of $2 M_{\odot}$ where the ${ }^{13} \mathrm{C}(\alpha, n){ }^{16} \mathrm{O}$ reaction is the main source of neutrons. We also examine an intermediate-mass AGB model of $5 M_{\odot}$ where intershell temperatures are high enough to activate the ${ }^{22} \mathrm{Ne}$ neutron source, which produces high neutron densities up to $\sim 10^{14} n \mathrm{~cm}^{-3}$. Hot bottom burning is activated in models with $M \geqslant 3 M_{\odot}$. With the $3 M_{\odot}$ model, we investigate the effect of varying the extent in mass of the region where protons are mixed from the envelope into the intershell at the deepest extent of each third dredge-up. We compare the results of the low-mass models to three post-AGB stars with a metallicity of $[\mathrm{Fe} / \mathrm{H}] \simeq-1.2$. The composition is a good match to the predicted neutron-capture abundances except for $\mathrm{Pb}$ and we confirm that the observed $\mathrm{Pb}$ abundances are lower than what is calculated by AGB models.
\end{abstract}

Key words: nuclear reactions, nucleosynthesis, abundances - stars: abundances - stars: AGB and post-AGB

Online-only material: color figures, machine-readable tables

\section{INTRODUCTION}

Stars with an initial mass of between $\sim 0.8$ and $\sim 8 M_{\odot}$, depending on initial metallicity, evolve through the asymptotic giant branch (AGB) phase. This is the last stage of nuclear burning for these stars (for a review, see Herwig 2005; Straniero et al. 2006; Karakas \& Lattanzio 2014). AGB stars are an observationally confirmed site for the slow neutron-capture process (the $s$-process, e.g., Abia et al. 2001), which is responsible for the production of around half of the abundance of the heavy elements beyond $\mathrm{Fe}$ (Gallino et al. 1998). AGB stars also produce a number of light elements such as lithium (e.g., Ventura \& D'Antona 2010), carbon (e.g., Izzard et al. 2009), fluorine (e.g., Abia et al. 2010; Recio-Blanco et al. 2012), and nitrogen (e.g., Johnson et al. 2007). Through nucleosynthesis and strong mass loss, AGB stars contribute to the chemical evolution of galaxies (Meléndez \& Cohen 2007; Romano et al. 2010; Letarte et al. 2010; Kobayashi et al. 2011) as well as globular clusters (Ventura \& D'Antona 2008; Meléndez \& Cohen 2009; Marino et al. 2011).

The stellar structure of an AGB star consists of an electron degenerate $\mathrm{CO}$ core surrounded by a He-burning shell and a $\mathrm{H}$-burning shell. These shells are separated by the He-intershell, which consists of approximately $75 \%{ }^{4} \mathrm{He}, 22 \%{ }^{12} \mathrm{C}$, and $2 \%{ }^{16} \mathrm{O}$ left over from partial He-burning. Surrounding the $\mathrm{H}$-exhausted core (hereafter core) is a large convective envelope. Neutroncapture nucleosynthesis via the $s$-process takes place in the He-intershell where the abundance of ${ }^{4} \mathrm{He}$ is high and $(\alpha, n)$ reactions can be efficiently activated releasing free neutrons that are then captured by the abundant ${ }^{56} \mathrm{Fe}$ seed nuclei. The $s$-process terminates at $\mathrm{Pb}$ and $\mathrm{Bi}$, the heaviest stable elements that can be produced with the low neutron densities that occur in AGB stars. For a review on $s$-process nucleosynthesis in AGB stars, see Busso et al. (1999).
During the thermally pulsing AGB phase, the star undergoes periodic thermal pulses (TPs) caused by instabilities in the thin He-burning shell. In order to liberate the energy that accumulates during He-burning, the He-burning shell drives a pulse-driven convective zone, which mixes ashes from the Heburning shell into the He-intershell. The energy released results in an expansion of the stellar layers above the $\mathrm{CO}$ core that effectively extinguishes the H-burning shell. This allows the convective envelope to move inward in mass. If the convective envelope moves into the He-intershell, material enriched from partial He-burning and $s$-process nucleosynthesis is mixed to the surface. This mixing mechanism is known as the third dredgeup (TDU) and is one way of altering the surface composition of an AGB star. Another important product that is mixed into the envelope is ${ }^{12} \mathrm{C}$ from partial He-burning. Therefore, the TDU is responsible for increasing the surface $\mathrm{C} / \mathrm{O}$ ratio with the possibility of creating carbon-rich stars that have a $\mathrm{C} / \mathrm{O}$ ratio greater than unity.

Nucleosynthesis in intermediate-mass AGB stars $\left(M \geqslant 3 M_{\odot}\right.$ at $Z=0.001$ ) can also occur via proton captures at the base of the convective envelope. This mechanism is known as hot bottom burning (HBB). The temperature at the base of the convective envelope becomes sufficiently high which activates $\mathrm{H}$-burning via the $\mathrm{CNO}$ cycle. If the temperature increases further, the $\mathrm{Ne}-\mathrm{Na}$ chain and $\mathrm{Mg}-\mathrm{Al}$ chain can also be activated (Arnould et al. 1999). One important consequence of $\mathrm{HBB}$ is the production of ${ }^{14} \mathrm{~N}$ at the expense of ${ }^{12} \mathrm{C}$ and ${ }^{16} \mathrm{O}$, as well as decreasing the $\mathrm{C} / \mathrm{O}$ ratio.

There are two main neutron source reactions in AGB stars: ${ }^{13} \mathrm{C}(\alpha, n){ }^{16} \mathrm{O}$ and ${ }^{22} \mathrm{Ne}(\alpha, n){ }^{25} \mathrm{Mg}$. The ${ }^{22} \mathrm{Ne}$ neutron source is efficiently activated at temperatures higher than approximately $300 \times 10^{6} \mathrm{~K}$. These temperatures are easily attained in the convective region that develops in the intershell during a TP for intermediate-mass stars. For low-mass stars, the ${ }^{22} \mathrm{Ne}$ neutron 
source is only marginally activated and is ineffective in producing the neutrons required for substantial $s$-process nucleosynthesis. However, the ${ }^{13} \mathrm{C}(\alpha, n){ }^{16} \mathrm{O}$ reaction is activated at temperatures as low as $90 \times 10^{6} \mathrm{~K}$, which means it can be ignited in low-mass stars (Straniero et al. 1995). In canonical stellar models there is not enough ${ }^{13} \mathrm{C}$ from the ashes of $\mathrm{H}$ burning for it to be an efficient source of neutrons. In order to increase the abundance of ${ }^{13} \mathrm{C}$ in the He-intershell, it is hypothesized that extra mixing of protons occurs at the deepest extent of the convective envelope during TDU. This is when a sharp composition discontinuity forms where the H-rich envelope and He-intershell meet. Protons that have been mixed downward are captured by ${ }^{12} \mathrm{C}$ forming a "pocket" of ${ }^{13} \mathrm{C}$ that usually burns in radiative conditions during the interpulse via the ${ }^{13} \mathrm{C}(\alpha, n){ }^{16} \mathrm{O}$ reaction before the next TP. This releases free neutrons at densities of $\lesssim 10^{8} n \mathrm{~cm}^{-3}$; much lower than the neutron densities reached by the ${ }^{22} \mathrm{Ne}$ source of up to $\sim 10^{14} n \mathrm{~cm}^{-3}$. The total number of neutrons released (the neutron exposure), however, is higher for the ${ }^{13} \mathrm{C}$ neutron source than the ${ }^{22} \mathrm{Ne}$ neutron source because the neutron flux lasts for roughly $10^{4} \mathrm{yr}$. For lowmass AGB stars, the ${ }^{13} \mathrm{C}$ pocket is responsible for producing the bulk of the abundances of the $s$-process elements (e.g., Bisterzo et al. 2014).

The AGB phase terminates once the stellar envelope has been ejected as a result of strong mass loss with the $\mathrm{CO}$ core remaining as a white dwarf. The ejected material enriches the interstellar medium from which the next generation of stars form.

The aim of this paper is to provide a self-consistent set of low- and intermediate-mass AGB models with $[\mathrm{Fe} / \mathrm{H}]^{3}=-1.2$ appropriate for the study of dwarf spheroidal galaxies and globular clusters as well as direct comparison to post-AGB stars. The models can also provide input for synthetic and parametric studies (e.g., Izzard et al. 2004). The models presented here are also applicable to investigating the pollution of Galactic halo stars by AGB stars and studies of galactic chemical evolution. We present evolution and nucleosynthesis results, including neutron-capture elements, for AGB models of 1 to $7 M_{\odot}$ for an initial metallicity of $[\mathrm{Fe} / \mathrm{H}]=-1.2$. The models presented here cover the most extensive mass range of $\mathrm{AGB}$ stars at $[\mathrm{Fe} / \mathrm{H}]=$ -1.2. In Section 2, we present the numerical details required for calculating the AGB stellar models. In Section 3, we present the stellar evolution results. The calculated models presented here provide the first detailed study of the TDU for an extended grid of AGB stars from 1 to $7 M_{\odot}$ at $[\mathrm{Fe} / \mathrm{H}]=-1.2$. In Section 4 , we explore in more detail the evolution and nucleosynthesis results of a typical low-mass model $\left(2 M_{\odot}\right)$ and a typical intermediatemass model ( $\left.5 M_{\odot}\right)$. In Section 5, we present the nucleosynthesis results including final surface abundances and stellar yields. In Section 6, we present the effect of varying the extent in mass of the region where protons are mixed from the envelope into the intershell for the $3 M_{\odot}$ model. In Section 7 , we present a comparison between the low-mass model predictions and the observed abundances of three post-AGB stars. In Section 8, we discuss uncertainties in the stellar abundances and stellar yields as a result of assumptions in the input physics and we end with discussion and conclusions in Section 9.

\section{EVOLUTIONARY AND NUCLEOSYNTHESIS CODES}

We calculate AGB stellar models for a range of initial masses from $1 M_{\odot}$ to $7 M_{\odot}$ with a metallicity of $Z=0.001$

$[\mathrm{X} / \mathrm{Y}]=\log _{10}\left(N_{X} / N_{Y}\right)_{\star}-\log _{10}\left(N_{X} / N_{Y}\right)_{\odot}$, where $N_{X}$ and $N_{Y}$ are the abundances of elements $\mathrm{X}$ and $\mathrm{Y}$.
$([\mathrm{Fe} / \mathrm{H}]=-1.2)$ and a helium abundance of $Y=0.25$. For the purposes of this study, we define the low-mass models to be those with an initial mass up to and including $3 M_{\odot}$, and the intermediate-mass models, $3.25 M_{\odot}$ and above. Each stellar model is evolved from the zero-age main sequence to near the end of the AGB phase when the majority of the convective envelope is lost by stellar winds. A two-step procedure is performed to calculate the structure and detailed nucleosynthesis for each stellar model.

First, we use the Mt Stromlo Stellar Evolutionary code (Karakas et al. 2010 and references therein) to calculate the stellar evolutionary sequences. The details of the procedure and evolution code are as described in Karakas et al. (2010) except for the differences described below. For the low-mass models, we use the $\mathrm{C}$ - and $\mathrm{N}$-enhanced $Æ S O P U S$ low-temperature molecular opacity tables (Marigo \& Aringer 2009) as used in Kamath et al. (2012). For the intermediate-mass models, we use updated Lodders (2003) scaled-solar ÆSOPUS lowtemperature molecular opacity tables (Marigo \& Aringer 2009), which account for the depletion and enhancement of $\mathrm{C}$ and $\mathrm{C} / \mathrm{O}$. The opacity treatment utilized for the intermediate-mass models is described in detail in Fishlock et al. (2014). We use OPAL tables (Iglesias \& Rogers 1996) updated to a Lodders (2003) scaled-solar abundance for consistency with the lowtemperature opacity tables.

To model convective borders, we follow the method described by Lattanzio (1986) and Frost \& Lattanzio (1996), which employs the Schwarzschild criterion but searches for a neutral border when $\nabla_{\text {ad }} / \nabla_{\text {rad }}$, the ratio of the adiabatic and radiative temperature gradients, is discontinuous such as during TDU. For convective regions, we use the standard mixing length theory (Böhm-Vitense 1958) with a mixing length parameter of $\alpha=1.86$. We use a solar global metallicity of $Z_{\odot}=0.015$ with a scaled-solar initial composition from Asplund et al. (2009) which has a protosolar metallicity of 0.0142 . As with Karakas et al. (2010), mass loss prior to the AGB phase is included using the Reimers (1975) formula with $\eta_{R}=0.4$. Mass loss during the AGB phase is included using the Vassiliadis \& Wood (1993) mass loss prescription.

Second, detailed nucleosynthesis calculations are performed using the stellar evolutionary sequences as input into a postprocessing nucleosynthesis code (see Lugaro et al. 2004, 2012 and references therein for details). The nucleosynthesis code calculates nuclear reactions and mixing simultaneously to solve for the abundances. A post-processing code is necessary as the stellar evolutionary code only accounts for the major energy generating reactions involving $\mathrm{H},{ }^{3} \mathrm{He},{ }^{4} \mathrm{He},{ }^{12} \mathrm{C},{ }^{14} \mathrm{~N}$, and ${ }^{16} \mathrm{O}$. We assume the additional reactions included in the postprocessing code produce negligible energy and do not affect the stellar structure (see Doherty et al. 2014a).

The updated nuclear network incorporated into the nucleosynthesis code is based on the JINA Reaclib ${ }^{4}$ database as of 2012 May with the modifications as detailed in Lugaro et al. (2014). The reaction rate of ${ }^{13} \mathrm{C}(\alpha, n){ }^{16} \mathrm{O}$ is taken from Heil et al. (2008) while the reaction rates for ${ }^{22} \mathrm{Ne}(\alpha, n)^{25} \mathrm{Mg}$ and ${ }^{22} \mathrm{Ne}(\alpha, \gamma){ }^{26} \mathrm{Mg}$ are taken from Iliadis et al. (2010). The network, which considers 2336 reactions, includes 320 species from neutrons to polonium and comprises all the stable and unstable isotopes relevant for $s$-process nucleosynthesis (for example, we do not include the long-lived isotope ${ }^{130} \mathrm{Te}$, because it is not reached by the $s$-process). We further include two species

\footnotetext{
4 https://groups.nscl.msu.edu/jina/reaclib/db/
} 
Table 1

Evolutionary Properties of the Calculated $Z=0.001$ Stellar Models

\begin{tabular}{|c|c|c|c|c|c|c|c|c|c|}
\hline $\begin{array}{l}M_{\text {initial }}{ }^{\mathrm{a}} \\
\left(M_{\odot}\right)\end{array}$ & $\begin{array}{c}M_{\text {final }}{ }^{\mathrm{b}} \\
\left(M_{\odot}\right)\end{array}$ & $\begin{array}{c}M_{\text {core }}{ }^{\mathrm{c}} \\
\left(M_{\odot}\right)\end{array}$ & $\begin{array}{l}M_{\text {env }}{ }^{\mathrm{d}} \\
\left(M_{\odot}\right)\end{array}$ & TPs ${ }^{\mathrm{e}}$ & TDUs $^{\mathrm{f}}$ & $\lambda_{\max } \mathrm{g}$ & $\begin{array}{c}T_{\mathrm{BCE}}^{\max \mathrm{h}} \\
\left(10^{6} \mathrm{~K}\right)\end{array}$ & $\begin{array}{c}T_{\mathrm{He}}^{\max \mathrm{i}} \\
\left(10^{6} \mathrm{~K}\right)\end{array}$ & $\begin{array}{r}M_{\mathrm{TDU}}{ }^{\mathrm{j}} \\
\left(M_{\odot}\right)\end{array}$ \\
\hline 1 & 0.678 & 0.667 & 0.011 & 17 & 2 & 0.08 & 1.4 & 284.1 & 0.002 \\
\hline 1.25 & 0.669 & 0.649 & 0.020 & 14 & 8 & 0.16 & 2.2 & 271.8 & 0.009 \\
\hline 1.5 & 0.657 & 0.646 & 0.011 & 14 & 10 & 0.37 & 6.7 & 275.4 & 0.026 \\
\hline 2 & 0.668 & 0.661 & 0.007 & 17 & 14 & 0.73 & 4.2 & 294.2 & 0.095 \\
\hline 2.25 & 0.839 & 0.673 & 0.166 & 17 & 16 & 0.82 & 5.6 & 305.4 & 0.132 \\
\hline 2.5 & 0.948 & 0.709 & 0.239 & 17 & 16 & 0.92 & 9.2 & 318.5 & 0.138 \\
\hline 2.75 & 1.057 & 0.746 & 0.312 & 18 & 18 & 0.97 & 15.8 & 320.8 & 0.138 \\
\hline 3 & 1.189 & 0.792 & 0.397 & 22 & 20 & 1.00 & 28.3 & 332.5 & 0.124 \\
\hline 3.25 & 1.403 & 0.843 & 0.561 & 23 & 22 & 1.00 & 48.9 & 350.5 & 0.093 \\
\hline 3.5 & 1.176 & 0.857 & 0.319 & 27 & 27 & 0.99 & 58.5 & 361.3 & 0.104 \\
\hline 4 & 1.726 & 0.883 & 0.843 & 68 & 68 & 1.02 & 82.9 & 361.3 & 0.231 \\
\hline 4.5 & 1.659 & 0.908 & 0.750 & 79 & 78 & 0.97 & 87.6 & 356.6 & 0.210 \\
\hline 5 & 1.740 & 0.938 & 0.802 & 94 & 93 & 0.95 & 92.5 & 361.2 & 0.194 \\
\hline 5.5 & 1.962 & 0.972 & 0.990 & 100 & 99 & 0.93 & 98.1 & 363.0 & 0.151 \\
\hline 6 & 1.725 & 1.015 & 0.709 & 108 & 105 & 0.92 & 104.8 & 376.5 & 0.107 \\
\hline 7 & 2.062 & 1.145 & 0.917 & 135 & 132 & 0.86 & 125.0 & 392.4 & 0.034 \\
\hline
\end{tabular}

Notes.

${ }^{\text {a }}$ Initial mass.

b Final mass.

c Final core mass.

${ }^{\mathrm{d}}$ Final envelope mass.

e Number of TPs computed.

${ }^{f}$ Number of TDU episodes.

g Maximum efficiency of TDU.

${ }^{\mathrm{h}}$ Maximum temperature reached at the base of the convective envelope.

${ }^{\mathrm{i}}$ Maximum temperature reached at the base of the He-intershell.

$\mathrm{j}$ Total amount of mass mixed into the envelope through TDU.

for the unstable isotope ${ }^{85} \mathrm{Kr}$, the ground state ${ }^{85} \mathrm{Kr}^{g}$ and the short-lived metastable state ${ }^{85} \mathrm{Kr}^{m}$, due to their location at an $s$ process branching point. When determining surface abundances and yields, we assume that long-lived isotopes have decayed (e.g., ${ }^{99} \mathrm{Tc}$ to ${ }^{99} \mathrm{Ru}$ ).

For the low-mass models, a partial mixing zone (PMZ) is included in the post-processing nucleosynthesis code. Protons are artificially added to the top layers of the He-intershell at the deepest extent of TDU where they are captured by ${ }^{12} \mathrm{C}$ leading to the production of the ${ }^{13} \mathrm{C}$ pocket (see Lugaro et al. 2012). This produces the free neutrons required for $s$-process nucleosynthesis. For the low-mass models with an initial mass less than $2.75 M_{\odot}$, we choose the mass of the added PMZ to be $2 \times 10^{-3} M_{\odot}$. For the 2.75 and $3 M_{\odot}$ models, we choose a PMZ mass of $1 \times 10^{-3} M_{\odot}$ and $5 \times 10^{-4} M_{\odot}$, respectively. We choose a lower PMZ mass for the 2.75 and $3 M_{\odot}$ models because of the effect of a decreasing intershell mass with initial mass. We discuss the uncertainty related to the choice of the PMZ mass in Section 6. We set the mass to remain constant for every PMZ added during TDU. As the intershell mass reduces with each TP we take the neutron-capture abundances to be an upper limit.

\section{STELLAR EVOLUTION RESULTS}

In Table 1, we provide a summary of the structural properties relevant for nucleosynthesis for each of the AGB models. We provide online tables for each model which include structural properties for each TP. Each online table includes the pulse number, core mass, maximum mass of the intershell convection zone, duration of intershell convection, mass dredged into the envelope, the TDU efficiency, maximum temperature in the He-shell, maximum temperature at the base of the convective envelope during the previous interpulse period, maximum temperature in the $\mathrm{H}$-shell during the previous interpulse period, interpulse period, total mass, maximum radiated luminosity during the interpulse period, maximum He-luminosity during a TP, maximum radius during the previous interpulse period, bolometric magnitude, and effective temperature at maximum radius. Table 2 shows a portion of the table for each TP of the $1 M_{\odot}$ model and is published in its entirety in the online journal.

The AGB phase is terminated when the stellar envelope is removed through mass loss. The low-mass models, excluding the $1 M_{\odot}$ model, experience the superwind phase in the final few TPs during which the mass-loss rate reaches a plateau of approximately $10^{-5} M_{\odot} \mathrm{yr}^{-1}$. The $1 M_{\odot}$ model loses the majority of its stellar envelope before it reaches the superwind phase. The intermediate-mass models experience the superwind phase well before most of the envelope has been lost.

For models with an initial mass up to (and including) $2 M_{\odot}$, we are able to evolve the envelope mass to less than $0.02 M_{\odot}$, which puts the model just beyond the tip of the AGB toward the post-AGB phase (Blöcker 2001). The models with $M \gtrsim 2 M_{\odot}$ suffer from convergence problems toward the end of the AGB (see Lau et al. 2012, for more details). For the models between 2 and $4 M_{\odot}$, we are able to evolve the envelope mass to less than $0.6 M_{\odot}$. However, for the models between $4 M_{\odot}$ and $7 M_{\odot}$, we are able to evolve the envelope mass to less than $1 M_{\odot}$.

Since some envelope mass still remains, it is possible that additional TDU episodes could occur that would further enrich the envelope prior to being ejected into the interstellar medium (see Karakas \& Lattanzio 2007). If we assume that the mass lost during the final calculated interpulse period is taken as 
Table 2

An Example of the Online Data Table Providing the Structural Properties for Each TP of the $1 M_{\odot}$ Model

\begin{tabular}{|c|c|c|c|c|c|c|c|c|c|c|c|c|c|c|c|c|}
\hline $\begin{array}{l}M_{\mathrm{i}}^{\mathrm{a}} \\
\left(M_{\odot}\right)\end{array}$ & $\mathrm{TP}^{\mathrm{b}}$ & $\begin{array}{l}M_{\text {core }}{ }^{\mathrm{c}} \\
\left(M_{\odot}\right)\end{array}$ & $\begin{array}{l}M_{\mathrm{csh}^{\mathrm{d}}} \\
\left(M_{\odot}\right)\end{array}$ & $\begin{array}{l}\tau_{\mathrm{csh}}{ }^{\mathrm{e}} \\
(\mathrm{yr})\end{array}$ & $\begin{array}{c}M_{\text {dredge }}{ }^{f} \\
\left(M_{\odot}\right)\end{array}$ & $\lambda^{\mathrm{g}}$ & $\begin{array}{c}T_{\mathrm{He}}^{\max \mathrm{h}} \\
(\mathrm{K})\end{array}$ & $\begin{array}{c}T_{\mathrm{TBCE}}^{\max } \mathrm{i} \\
(\mathrm{K})\end{array}$ & $\begin{array}{c}T_{\mathrm{He}}^{\max , \mathrm{ip} j} \\
\text { (K) }\end{array}$ & $\begin{array}{l}\tau_{\mathrm{ip}}{ }^{\mathrm{k}} \\
(\mathrm{yr})\end{array}$ & $\begin{array}{l}M_{\mathrm{tot}}{ }^{1} \\
\left(M_{\odot}\right)\end{array}$ & $\begin{array}{l}L_{\max }{ }^{m} \\
\left(L_{\odot}\right)\end{array}$ & $\begin{array}{c}L_{\mathrm{He}}^{\max \mathrm{n}} \\
\left(L_{\odot}\right)\end{array}$ & $\begin{array}{c}R_{\max }{ }^{o} \\
\left(R_{\odot}\right)\end{array}$ & $\begin{array}{l}m_{\text {bol }}{ }^{p} \\
(\mathrm{mag})\end{array}$ & $\begin{array}{l}T_{\text {eff }}{ }^{\mathrm{q}} \\
(\mathrm{K})\end{array}$ \\
\hline 0 & 1 & $9 e-1$ & $8 e-2$ & $2 e+2$ & $00 \mathrm{e}+0$ & +0 & +8 & $18 \mathrm{e}+6$ & $5.32 \mathrm{e}+7$ & $0 \mathrm{e}+0$ & $9.03 \mathrm{e}-1$ & $.58 \mathrm{e}+3$ & $2.43 \mathrm{e}+5$ & $7.98 \mathrm{e}+1$ & -0 & 4 \\
\hline 0 & 2 & $e-1$ & $6 e-2$ & $18 \mathrm{e}+2$ & $e+0$ & $e+0$ & +8 & +6 & +7 & $9 e+5$ & $e-1$ & +3 & +5 & +1 & +0 & \\
\hline .0 & 3 & $e-1$ & $21 e-2$ & $5.30 \mathrm{e}+2$ & $\mathrm{e}+0$ & $0.00 \mathrm{e}+0$ & $27 e+8$ & $0 e+6$ & $5.86 \mathrm{e}+7$ & $17 \mathrm{e}+5$ & $9.03 \mathrm{e}-1$ & $55 e+3$ & $66 e+6$ & $1 e+2$ & & $e+3$ \\
\hline 1.0 & 4 & $5.47 \mathrm{e}-1$ & $97 e-2$ & $4.80 \mathrm{e}+2$ & $0.00 \mathrm{e}+0$ & $0.00 \mathrm{e}+0$ & $37 e+8$ & $1.34 \mathrm{e}+6$ & $6.05 \mathrm{e}+7$ & $32 \mathrm{e}+5$ & $9.03 \mathrm{e}-1$ & & $8.18 \mathrm{e}+6$ & & & $92 \mathrm{e}+3$ \\
\hline .0 & 5 & $5.57 \mathrm{e}-1$ & $3.74 \mathrm{e}-2$ & $4.85 e+2$ & $0.00 \mathrm{e}+0$ & $0.00 \mathrm{e}+0$ & $2.45 \mathrm{e}+8$ & $1.38 \mathrm{e}+6$ & $6.23 e+7$ & $3.12 \mathrm{e}+5$ & $9.03 e-1$ & $5 e+3$ & $1 e+7$ & $8 \mathrm{e}+2$ & $e+0$ & $3.88 \mathrm{e}+3$ \\
\hline .0 & 6 & $5.67 e-1$ & $3.48 \mathrm{e}-2$ & $3.97 \mathrm{e}+2$ & $0.00 \mathrm{e}+0$ & $0.00 \mathrm{e}+0$ & $2.52 \mathrm{e}+8$ & & $6.38 \mathrm{e}+7$ & $2.84 e+5$ & $3 e-1$ & & $3 e+7$ & & -4 & \\
\hline 1.0 & 7 & $5.77 \mathrm{e}-1$ & $3.24 \mathrm{e}-2$ & $3.86 \mathrm{e}+2$ & 0.00 & +0 & 2.57 & $1.43 e+6$ & 6.5 & $2.53 e+5$ & & & & & -4 & \\
\hline 1.0 & 8 & $5.88 \mathrm{e}-1$ & $3.02 \mathrm{e}-2$ & $3.44 \mathrm{e}+2$ & 0.00 & 0.00 & 2.6 & 1.43 & 6.6 & 2.2 & -1 & & & & -4 & \\
\hline 1.0 & 9 & $5.98 \mathrm{e}-1$ & $2.80 \mathrm{e}-2$ & $2.95 e+2$ & $7.84 e-4$ & $7.56 \mathrm{e}-2$ & 2.66 & 1.42 & 6.77 & 1.99 & 9.0 & & & & -4 & \\
\hline 1.0 & 10 & $6.07 \mathrm{e}-1$ & $2.59 \mathrm{e}-2$ & $2.52 \mathrm{e}+2$ & $7.36 e-4$ & $7.30 \mathrm{e}-2$ & $2.67 \mathrm{e}+8$ & $1.33 \mathrm{e}+6$ & $6.73 e+7$ & $1.66 \mathrm{e}+5$ & $8.97 \mathrm{e}-1$ & 5.99 & 4.04 & $2.10 \mathrm{e}+2$ & -4.7 & 3.6 \\
\hline 0 & 11 & $e-1$ & $e-2$ & $1.98 \mathrm{e}+2$ & 0.0 & +0 & 2 & 1. & 6.7 & -5 & -1 & 6.5 & 4. & 2. & - & \\
\hline .0 & 12 & -1 & $e-2$ & $2.04 \mathrm{e}+2$ & +0 & -0 & 2.7 & 1. & 6.8 & -5 & -1 & & 4. & 2.5 & - & \\
\hline .0 & 13 & $e-1$ & $e-2$ & $1.87 \mathrm{e}+2$ & +0 & & 2. & 8.4 & 6.8 & +5 & -1 & $1 .{ }^{4}$ & 4.2 & 2.6 & -4 & \\
\hline .0 & 14 & $6.43 e-1$ & $1.96 \mathrm{e}-2$ & $1.74 \mathrm{e}+2$ & $0.00 \mathrm{e}+0$ & $0.00 \mathrm{e}+0$ & $2.76 \mathrm{e}+8$ & $6.32 \mathrm{e}+5$ & $6.96 \mathrm{e}+7$ & $e+5$ & $7.36 \mathrm{e}-1$ & $7.79 e+3$ & $4.58 \mathrm{e}+7$ & $2.63 e+2$ & -5 & \\
\hline 1.0 & 15 & $6.51 \mathrm{e}-1$ & $1.85 \mathrm{e}-2$ & $1.62 \mathrm{e}+2$ & $0.00 \mathrm{e}+0$ & $0.00 \mathrm{e}+0$ & $2.78 \mathrm{e}+8$ & $3.88 \mathrm{e}+5$ & $7.04 \mathrm{e}+7$ & $9.59 \mathrm{e}+4$ & $6.95 \mathrm{e}-1$ & $8.17 \mathrm{e}+3$ & $4.98 \mathrm{e}+7$ & $2.39 \mathrm{e}+2$ & $-5.06 e+0$ & $3.69 \mathrm{e}+3$ \\
\hline 1.0 & 16 & $6.59 \mathrm{e}-1$ & $1.75 e-2$ & $1.56 \mathrm{e}+2$ & $0.00 \mathrm{e}+0$ & $0.00 \mathrm{e}+0$ & $2.81 \mathrm{e}+8$ & $2.59 \mathrm{e}+5$ & $7.12 \mathrm{e}+7$ & $8.78 \mathrm{e}+4$ & $6.81 \mathrm{e}-1$ & $8.56 \mathrm{e}+3$ & $5.25 \mathrm{e}+7$ & $2.16 \mathrm{e}+2$ & $-5.11 \mathrm{e}+0$ & $3.90 \mathrm{e}+3$ \\
\hline 1.0 & 17 & $6.67 \mathrm{e}-1$ & $1.66 \mathrm{e}-2$ & $1.53 \mathrm{e}+2$ & $0.00 \mathrm{e}+0$ & $0.00 \mathrm{e}+0$ & $2.84 \mathrm{e}+8$ & $2.14 \mathrm{e}+5$ & $7.19 \mathrm{e}+7$ & $8.02 \mathrm{e}+4$ & $6.78 \mathrm{e}-1$ & $8.96 \mathrm{e}+3$ & $5.54 \mathrm{e}+7$ & $1.87 \mathrm{e}+2$ & $-5.16 e+0$ & $4.30 \mathrm{e}+3$ \\
\hline
\end{tabular}

Notes.

${ }^{a}$ Initial stellar mass.

${ }^{b}$ Pulse number.

${ }^{\mathrm{c}}$ Core mass.

${ }^{\mathrm{d}}$ Maximum mass of the intershell convection zone.

e Duration of intershell convection.

${ }^{\mathrm{f}}$ Mass dredged into the envelope.

$\mathrm{g}$ The TDU efficiency.

${ }^{\mathrm{h}}$ Maximum temperature in the He-shell.

${ }^{i}$ Maximum temperature at the base of the convective envelope during the previous interpulse period.

${ }^{\mathrm{j}}$ Maximum temperature in the $\mathrm{H}$-shell during the previous interpulse period.

${ }^{\mathrm{k}}$ Interpulse period.

${ }^{1}$ Total mass.

${ }^{\mathrm{m}}$ Maximum radiated luminosity during the previous interpulse period.

${ }^{\mathrm{n}}$ Maximum He luminosity during a TP.

${ }^{\circ}$ Maximum radius during the previous interpulse period.

p Bolometric magnitude.

${ }^{\mathrm{q}}$ Effective temperature at maximum radius.

(This table is available in its entirety in a machine-readable form in the online journal. A portion is shown here for guidance regarding its form and content.)

representative of the mass to be lost before the next possible TP, the models with $M \leqslant 3.5 M_{\odot}$ cannot experience another TDU as there is not enough envelope mass left. The more massive models, however, retain sufficient mass to experience at least one more TDU episode. For example, the $6 M_{\odot}$ model has an envelope mass of $0.709 M_{\odot}$ remaining when calculations cease due to convergence issues. To estimate the number of remaining of TPs, we assume the mass lost during the preceding TP is taken as representative of the mass to be lost in the following TPs (approximately $1.5 \times 10^{-1} M_{\odot}$ ). This leaves a minimum of an additional four TPs (possibly with TDU) that could take place. We remove the remaining envelope without taking into account the possibility for extra TDU(s). Therefore, the final surface abundance and yield predictions of the neutron-capture elements are a lower limit for the intermediate-mass models. Additionally, the termination of the intermediate-mass models occurs after HBB has ceased.

In Table 3 we identify models that experience a core He-flash, the first dredge-up (FDU; after core H-burning at the base of the RGB), the second dredge-up (SDU; after core He-burning on the early AGB), TDU, and/or HBB. All the models calculated with an initial mass less than $2 M_{\odot}$ develop an electron degenerate
Table 3

A Tick $(\checkmark)$ Means the Phenomenon Occurred in Each Model, a Cross ( $\times$ ) If It Did Not

\begin{tabular}{lccccc}
\hline \hline Mass & Core He-flash & FDU & SDU & TDU & HBB \\
$\left.M_{\odot}\right)$ & & & & & \\
\hline 1 & $\checkmark$ & $\checkmark$ & $\times$ & $\checkmark$ & $\times$ \\
1.25 & $\checkmark$ & $\checkmark$ & $\times$ & $\checkmark$ & $\times$ \\
1.5 & $\checkmark$ & $\checkmark$ & $\times$ & $\checkmark$ & $\times$ \\
2 & $\times$ & $\checkmark$ & $\times$ & $\checkmark$ & $\times$ \\
2.25 & $\times$ & $\checkmark$ & $\times$ & $\checkmark$ & $\times$ \\
2.5 & $\times$ & $\checkmark$ & $\times$ & $\checkmark$ & $\times$ \\
2.75 & $\times$ & $\checkmark$ & $\checkmark$ & $\checkmark$ & $\times$ \\
3 & $\times$ & $\checkmark$ & $\checkmark$ & $\checkmark$ & $\checkmark$ \\
3.25 & $\times$ & $\checkmark$ & $\checkmark$ & $\checkmark$ & $\checkmark$ \\
3.5 & $\times$ & $\checkmark$ & $\checkmark$ & $\checkmark$ & $\checkmark$ \\
4 & $\times$ & $\times$ & $\checkmark$ & $\checkmark$ & $\checkmark$ \\
4.5 & $\times$ & $\times$ & $\checkmark$ & $\checkmark$ & $\checkmark$ \\
5 & $\times$ & $\times$ & $\checkmark$ & $\checkmark$ & $\checkmark$ \\
5.5 & $\times$ & $\times$ & $\checkmark$ & $\checkmark$ & $\checkmark$ \\
6 & $\times$ & $\times$ & $\checkmark$ & $\checkmark$ & $\checkmark$ \\
7 & $\times$ & $\times$ & $\checkmark$ & $\checkmark$ & $\checkmark$ \\
\hline
\end{tabular}



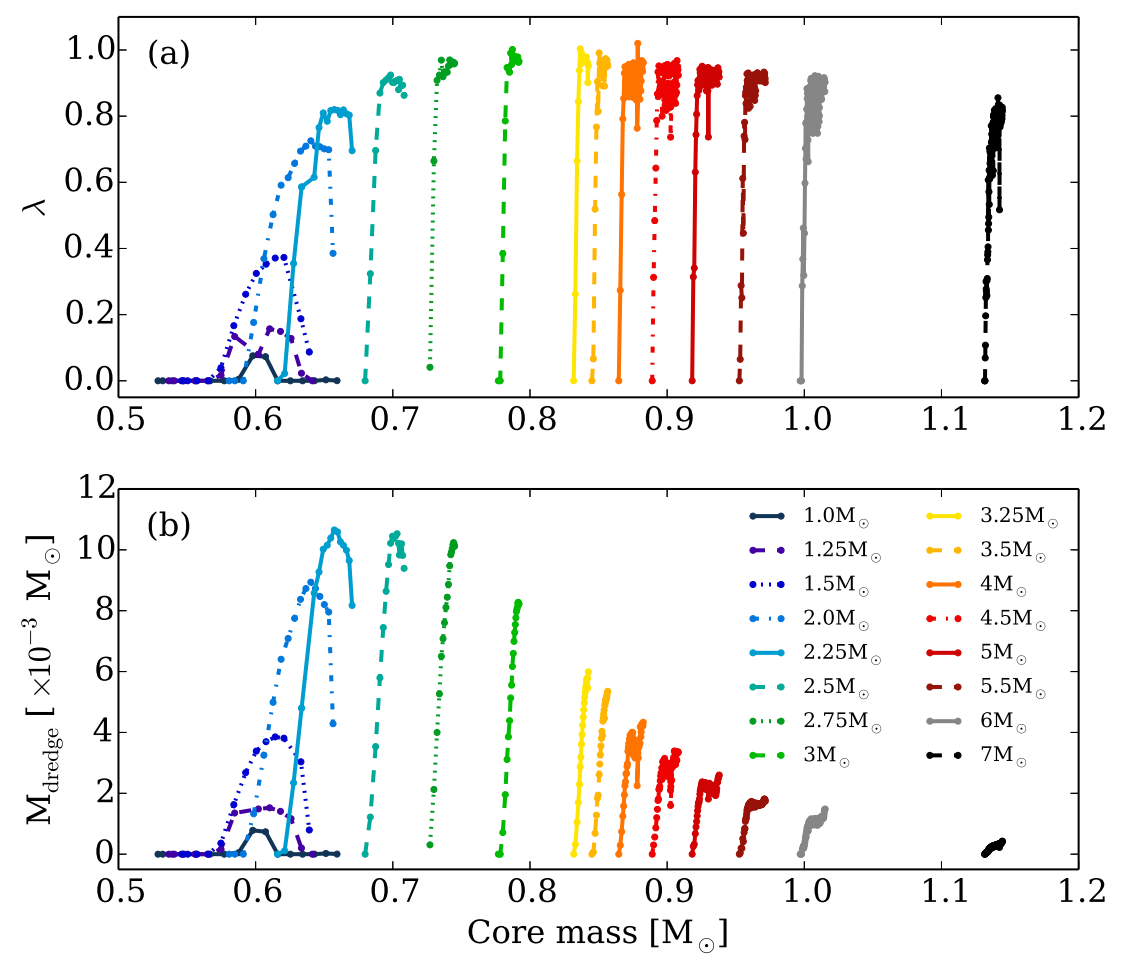

Figure 1. Evolution of (a) the efficiency of TDU, $\lambda$ and (b) $M_{\text {dredge }}$ with core mass for each model.

(A color version of this figure is available in the online journal.)

core and experience a core He-flash at the onset of core Heignition. A core He-flash removes this degeneracy and the luminosity of the $\mathrm{H}$-shell briefly reaches up to $10^{9} L_{\odot}$. FDU is experienced in all models $\leqslant 3.5 M_{\odot}$ (Table 3$)$. The intermediatemass models with $M \geqslant 4 M_{\odot}$ do not experience FDU as core He-burning is ignited before the model reaches the first giant branch; these stars experience SDU as their first mixing episode. We find that SDU occurs in models with $M \geqslant 2.75 M_{\odot}$. The 2.75 to $3.5 M_{\odot}$ models are the only models to experience both FDU and SDU.

All the stellar models experience TDU on the AGB and the efficiency of TDU is quantified by the parameter,

$$
\lambda=\frac{\Delta M_{\mathrm{dredge}}}{\Delta M_{\mathrm{c}}},
$$

where $\Delta M_{\text {dredge }}$ is the mass of the material mixed into the convective envelope by the TDU episode and $\Delta M_{\mathrm{c}}$ is the mass growth of the core due to $\mathrm{H}$-burning during the preceding interpulse period. The $1 M_{\odot}$ model experiences the fewest number of TDUs with only six episodes which brings a total of $0.0016 M_{\odot}$ of enriched material to the stellar surface. The largest total amount of material that is mixed to the surface is $0.231 M_{\odot}$, which occurs for the $4 M_{\odot}$ model. Despite having more TDU episodes than the $4 M_{\odot}$ model, the $4.5,5,5.5,6$, and $7 M_{\odot}$ models dredge up a smaller amount of material. This is because the intershell region is not as massive and TDU is less efficient in these models compared to the $4 M_{\odot}$ model.

Figure 1(a) illustrates the evolution of $\lambda$ with core mass for each model and the range of core masses produced by the models. The TDU efficiency gradually increases with increasing core mass. Overall, the efficiency of TDU increases with initial mass with the maximum $\lambda$ values occurring for the 3 and $3.25 M_{\odot}$ models. The overall efficiency then decreases for the $4.5,5,5.5,6$, and $7 M_{\odot}$ models. Figure 1 (b) reveals that the lowmass models, with the lowest $\lambda$ values, mix up more material per TDU as a result of a larger intershell mass compared to the intermediate-mass models. However, $M_{\text {dredge }}$ does not correlate with $\lambda$ value. The increase in the core mass during the AGB phase is higher for the low-mass models as a result of a low $\lambda$. The mass of the core of the $1 M_{\odot}$ model increases by $0.14 M_{\odot}$ while the core mass of the $7 M_{\odot}$ model only increases by $0.014 M_{\odot}$, a factor of 10 lower. This is a result of the high efficiency of TDU $(\lambda \approx 1)$ and shorter interpulse periods in the intermediate-mass models leading to minimal core growth.

The maximum temperature reached at the base of the convective envelope increases with increasing initial mass reaching up to $125 \times 10^{6} \mathrm{~K}$ for the $7 M_{\odot}$ model (see Table 1 ). While we find the lower initial stellar mass limit for HBB to be $3 M_{\odot}$ (Table 3), there is only mild activation of HBB for a few TPs in models less than $4 M_{\odot}$. The lower initial mass limit for efficient HBB with $T_{\text {bce }} \gtrsim 60 \times 10^{6} \mathrm{~K}$ is $4 M_{\odot}$. The $Z=0.001$ models with an initial mass $\leqslant 2.5 M_{\odot}$ of Ventura \& Marigo (2010) do not experience HBB where they define the onset of HBB to be $T_{\text {bce }} \gtrsim 60 \times 10^{6} \mathrm{~K}$.

The 6 and $7 M_{\odot}$ models experience hot TDU where HBB takes place during TDU as $\mathrm{C}$ and $\mathrm{O}$ is mixed the surface. The studies by Goriely \& Siess (2004) and Herwig (2004) demonstrated that hot TDU can inhibit $s$-process nucleosynthesis. For these models, and the other intermediate-mass models, we do not include a PMZ.

The $7 M_{\odot}$ model is characterized as a super-AGB star as it experiences off-center carbon ignition which produces an $\mathrm{ONe}$ core at the end of the AGB phase (Siess 2007). Super-AGB stars also experience high mass-loss rates with the $7 M_{\odot}$ model reaching a maximum rate of $1.3 \times 10^{-3} M_{\odot} \mathrm{yr}^{-1}$ after around 60 TPs. In comparison, the $6 M_{\odot}$ reaches a maximum mass-loss rate of $8.8 \times 10^{-4} M_{\odot} \mathrm{yr}^{-1}$ after around 80 TPs. The grid of superAGB models calculated by Doherty et al. (2014b) includes a $7 M_{\odot}$ model of $Z=0.001$. A comparison between these two models finds similar final core masses $\left(1.14 M_{\odot}\right.$ compared to 
Table 4

Properties of Each TP for the $2 M_{\odot}$ Model

\begin{tabular}{|c|c|c|c|c|c|c|c|c|c|}
\hline $\mathrm{TP}$ & $\begin{array}{c}M_{\mathrm{tot}} \\
\left(M_{\odot}\right)\end{array}$ & $\begin{array}{l}M_{\text {core }} \\
\left(M_{\odot}\right)\end{array}$ & $\begin{array}{c}M_{\text {dredge }} \\
\left(\times 10^{-3} M_{\odot}\right)\end{array}$ & $\lambda$ & $\begin{array}{c}\log _{10} \tau_{\text {ip }} \\
(\mathrm{yr})\end{array}$ & $\begin{array}{c}\log _{10} L_{\max } \\
\left(L_{\odot}\right)\end{array}$ & $\begin{array}{c}\log _{10} T_{\text {eff }} \\
(\mathrm{K})\end{array}$ & $\begin{array}{l}R_{\max } \\
\left(R_{\odot}\right)\end{array}$ & $\mathrm{C} / \mathrm{O}$ \\
\hline 1 & 1.9737 & 0.581 & 0.00 & 0.00 & $\ldots$ & 3.36 & 3.64 & 85.41 & 0.29 \\
\hline 2 & 1.9737 & 0.585 & 0.00 & 0.00 & 5.06 & 3.55 & 3.62 & 115.58 & 0.29 \\
\hline 3 & 1.9737 & 0.591 & 0.00 & 0.00 & 5.22 & 3.62 & 3.62 & 128.64 & 0.29 \\
\hline 4 & 1.9737 & 0.599 & 1.33 & 0.18 & 5.24 & 3.68 & 3.61 & 141.77 & 0.29 \\
\hline 5 & 1.9737 & 0.606 & 3.25 & 0.37 & 5.21 & 3.74 & 3.61 & 156.02 & 0.83 \\
\hline 6 & 1.9736 & 0.613 & 4.99 & 0.50 & 5.18 & 3.80 & 3.59 & 177.78 & 2.34 \\
\hline 7 & 1.9736 & 0.619 & 6.40 & 0.59 & 5.15 & 3.85 & 3.58 & 202.51 & 4.43 \\
\hline 8 & 1.9736 & 0.624 & 7.09 & 0.61 & 5.12 & 3.89 & 3.57 & 224.31 & 6.75 \\
\hline 9 & 1.9735 & 0.628 & 7.75 & 0.66 & 5.09 & 3.92 & 3.55 & 246.33 & 8.78 \\
\hline 10 & 1.9734 & 0.633 & 8.37 & 0.69 & 5.06 & 3.94 & 3.54 & 270.05 & 10.64 \\
\hline 11 & 1.9730 & 0.637 & 8.73 & 0.71 & 5.04 & 3.96 & 3.53 & 291.06 & 12.41 \\
\hline 12 & 1.9721 & 0.640 & 8.94 & 0.73 & 5.02 & 3.98 & 3.52 & 311.25 & 13.99 \\
\hline 13 & 1.9702 & 0.644 & 8.73 & 0.71 & 4.99 & 4.00 & 3.52 & 330.66 & 15.54 \\
\hline 14 & 1.9658 & 0.647 & 8.47 & 0.71 & 4.97 & 4.01 & 3.51 & 348.69 & 16.85 \\
\hline 15 & 1.9543 & 0.650 & 8.20 & 0.70 & 4.94 & 4.03 & 3.50 & 367.14 & 18.03 \\
\hline 16 & 1.9156 & 0.653 & 7.96 & 0.70 & 4.91 & 4.04 & 3.49 & 388.99 & 19.20 \\
\hline 17 & 1.3980 & 0.656 & 4.29 & 0.39 & 4.89 & 4.05 & 3.45 & 518.40 & 20.20 \\
\hline
\end{tabular}

Note. The final mass is given in Table 1 .

$1.145 M_{\odot}$ for the model presented here), maximum temperature at the base of the convective envelope (120 MK compared to $125 \mathrm{MK})$, and total mass of material dredged up $\left(3.97 \times 10^{-2} M_{\odot}\right.$ compared to $\left.3.4 \times 10^{-2} M_{\odot}\right)$. The $7 M_{\odot}$ model presented here experiences 135 TPs, whereas the Doherty et al. (2014b) model experiences 126 TPs and is evolved to a smaller envelope mass.

\section{DETAILS OF A LOW- AND INTERMEDIATE-MASS MODEL}

\subsection{The $2 M_{\odot}$ Model}

The $2 M_{\odot}$ model was chosen as a representative case of lowmass AGB evolution at $Z=0.001$ as we are able to compare our results with the calculations of Cristallo et al. (2009, 2011). Furthermore, we were able to evolve this model to a low-envelope mass of $0.007 M_{\odot}$. In Table 4 we present, for each TP, the total mass $\left(M_{\text {tot }}\right)$, the core mass $\left(M_{\text {core }}\right)$, the mass of material mixed to the surface due to TDU $\left(M_{\text {dredge }}\right)$, the efficiency of TDU $(\lambda)$, the interpulse period $\left(\tau_{\mathrm{ip}}\right)$, the maximum surface luminosity $\left(L_{\max }\right)$, the effective temperature $\left(T_{\text {eff }}\right)$, the maximum radius $\left(R_{\max }\right)$, and the surface $\mathrm{C} / \mathrm{O}$ ratio $(\mathrm{C} / \mathrm{O})$. The model experiences 17 TPs with 14 of these TPs followed by TDU.

In Figure 2(a), we plot the temporal evolution of three different mass boundaries during the AGB phase: the inner edge of the convective envelope, the mass of the H-exhausted core, and the mass of the He-exhausted core. The mass of the Heexhausted core remains constant during the interpulse because the He-burning shell is mostly inactive. It is only during a TP that the He-exhausted core increases in mass. The mass of the $\mathrm{H}$-exhausted core grows during the interpulse period when the $\mathrm{H}$-burning shell is active. Following a TP, the convective envelope moves inward in mass and, if TDU occurs, H-rich material is mixed into the $\mathrm{H}$-exhausted core thus reducing the mass of the core.

The first TDU episode occurs after the fourth TP (once $\lambda$ is greater than zero). The dredge-up efficiency increases for each successive TP until it reaches a maximum value of $\lambda=0.73$. TDU causes the $\mathrm{C} / \mathrm{O}$ ratio to increase above unity by the sixth $\mathrm{TP}$, changing the envelope composition from oxygen-rich to carbon-rich (see Table 4). The $\mathrm{C} / \mathrm{O}$ ratio is approximately 20 by the last TP. A total amount of $0.0945 M_{\odot}$ of enriched material is mixed into the envelope through TDU (Table 1), compared to $0.1313 M_{\odot}$ for the model calculated by Cristallo et al. (2011).

Appreciable envelope mass loss does not occur until the penultimate TP where the mass loss rate increases to approximately $2 \times 10^{-5} M_{\odot} \mathrm{yr}^{-1}$ during the superwind phase. The superwind phase is where the majority of the envelope, around $1 M_{\odot}$, is lost. This is shown along with the temporal evolution of total mass and core mass in Figures 2(b) and (c).

The composition profiles after the last TDU episode are presented in Figure 3 and focus on the region where the PMZ is added at the deepest extent of TDU. Key isotopes $\left(p,{ }^{12} \mathrm{C}\right.$, ${ }^{13} \mathrm{C},{ }^{16} \mathrm{O},{ }^{14} \mathrm{~N},{ }^{88} \mathrm{Sr},{ }^{138} \mathrm{Ba}$, and ${ }^{208} \mathrm{~Pb}$ ) involved in $s$-process nucleosynthesis are presented. When the convective envelope reaches its most inward point in mass during TDU, a PMZ of $2 \times 10^{-3} M_{\odot}$ is added to the top of the He-intershell (illustrated in Figure 3(a)). At the beginning of the interpulse, the ${ }^{13} \mathrm{C}$ pocket forms along with a pocket of ${ }^{14} \mathrm{~N}$. The mass of the ${ }^{13} \mathrm{C}$ pocket is approximately $1 \times 10^{-3} M_{\odot}$. Later, the ${ }^{13} \mathrm{C}(\alpha, n){ }^{16} \mathrm{O}$ reaction is activated increasing the neutron abundance. In the regions where the ${ }^{14} \mathrm{~N}$ abundance is higher than the ${ }^{13} \mathrm{C}$ abundance, no $s$-process nucleosynthesis can occur as ${ }^{14} \mathrm{~N}$ acts as a neutron poison via the ${ }^{14} \mathrm{~N}(n, p){ }^{14} \mathrm{C}$ reaction (illustrated in Figure 3(b)). Elements from the first peak, such as Sr, are produced first, followed by the second-peak elements such as $\mathrm{Ba} . \mathrm{Pb}$ is then produced at the expense of these elements (illustrated in Figure 3(c)). Eventually the abundance of ${ }^{13} \mathrm{C}$ reduces to below that of ${ }^{14} \mathrm{~N}$ and $s$-process nucleosynthesis terminates. The enriched material is then mixed into the following TP and then to the stellar surface through the next TDU.

Figure 4 highlights the distribution of the surface abundance ratios relative to $\mathrm{Fe}$ for all elements from $\mathrm{C}$ to $\mathrm{Bi}$. The "Initial" line is the initial composition on the main sequence. The "PreAGB" line is the pre-AGB composition as a result of FDU where the surface abundance of carbon, measured by $[\mathrm{C} / \mathrm{Fe}]$, decreases by 0.28 dex while $[\mathrm{N} / \mathrm{Fe}]$ increases by 0.49 dex. The ratio of $[\mathrm{Na} / \mathrm{Fe}]$ also increases by 0.22 dex. The remaining lines illustrate the surface abundances at the end of each TDU episode. The final abundances calculated by Cristallo et al. (2009) are also plotted. 

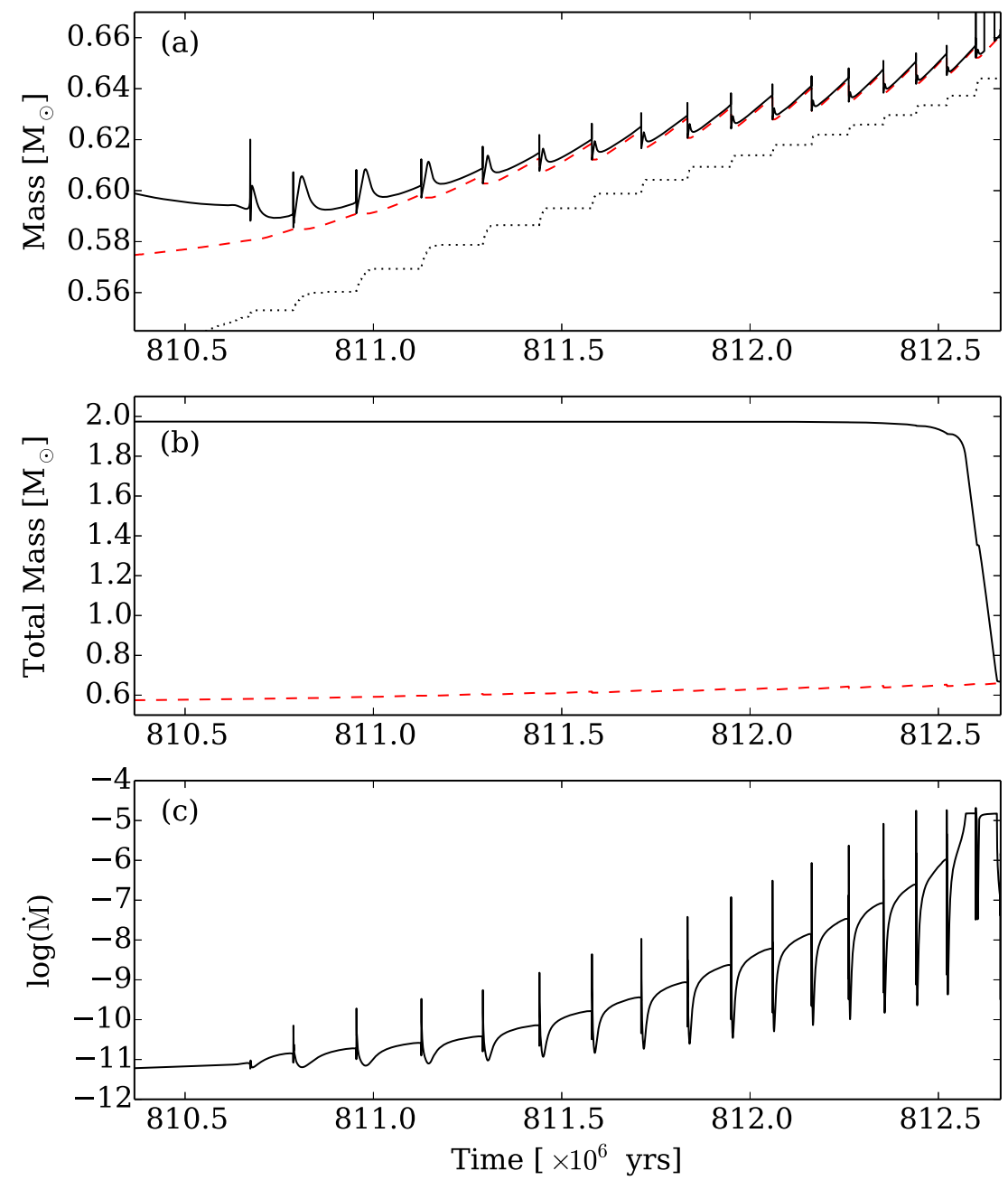

Figure 2. Temporal evolution from the start of the AGB phase for the $2 M_{\odot}$ model of (a) the inner edge of the convective envelope (solid line), the mass of the $\mathrm{H}$-exhausted core (dashed line) and the mass of the He-exhausted core (dotted line), (b) total mass (solid line) and the mass of the H-exhausted core (dashed line) and (c) the mass loss rate.

(A color version of this figure is available in the online journal.)
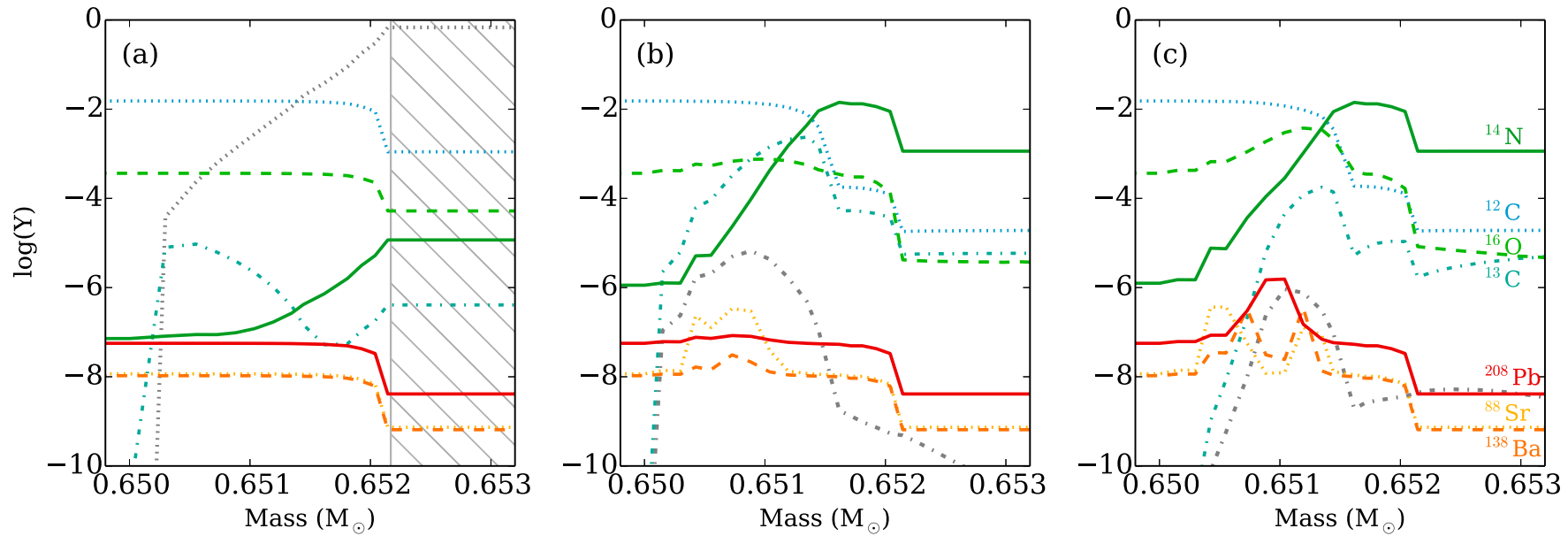

Figure 3. Composition profiles for three snapshots in the $2 M_{\odot}$ model after the last TDU. Abundances are given in units of $\log (Y)$, where $Y=X / A$ and $X$ is the mass fraction and $A$ is the atomic mass. Protons are shown by the gray dotted line. Neutrons are shown by the gray dash-dotted line and are offset in $\log (Y)$ by +15 . The hatched region represents the convective envelope. Panel (a): the proton profile of the added PMZ just after the deepest extent of the last TDU. Panel (b): the ${ }^{13} \mathrm{C}$ pocket has formed along with a ${ }^{14} \mathrm{~N}$ pocket with the ${ }^{13} \mathrm{C}(\alpha, n){ }^{16} \mathrm{O}$ reaction producing a peak of neutrons. The neutron-capture elements are starting to be synthesized. Panel (c): $\mathrm{Pb}$ is created at the expense of $\mathrm{Ba}$ and the abundance of ${ }^{13} \mathrm{C}$ is now below that of ${ }^{14} \mathrm{~N}$ so no more neutrons can be produced.

(A color version of this figure is available in the online journal.) 


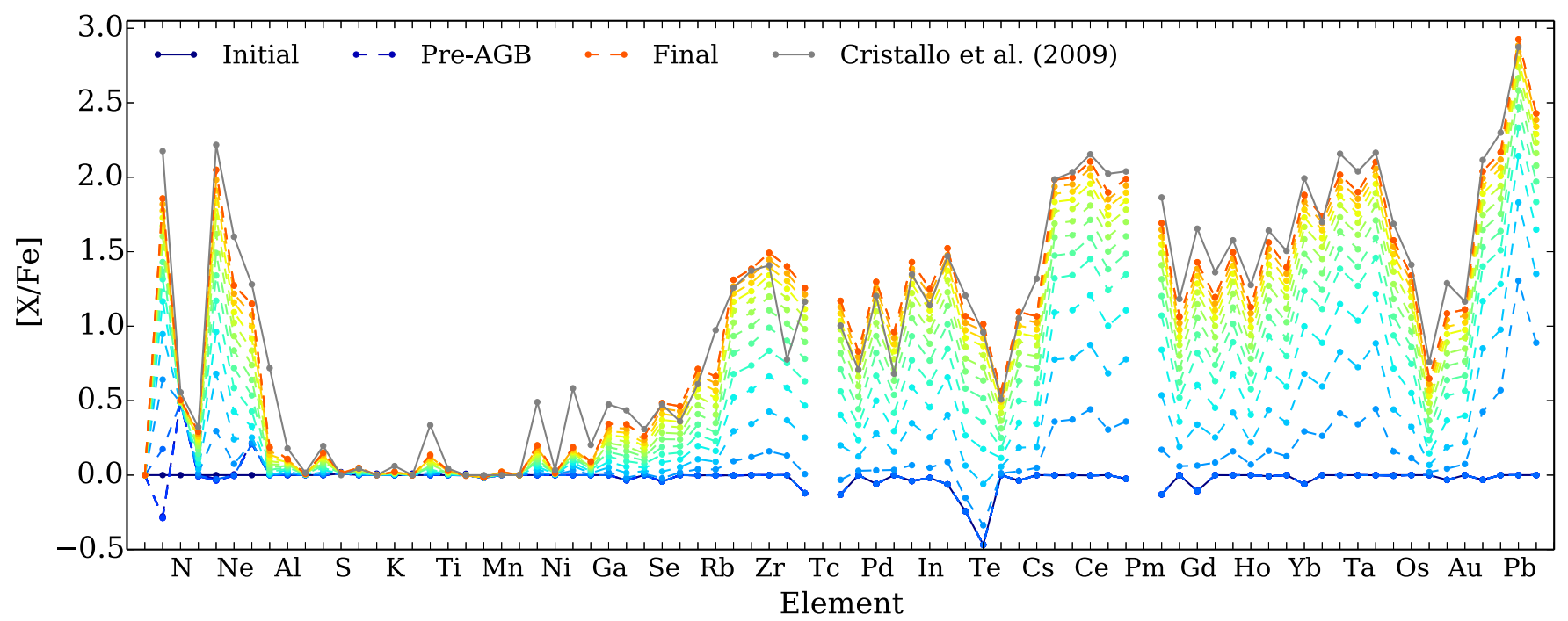

Figure 4. Surface abundance ratio for each of the elements after each TDU for the $2 M_{\odot}$ model. Each line connects the [X/Fe] abundance after each TDU. The initial and pre-AGB compositions are also shown. The initial composition shows that some elements have a value less than solar. This is a result of a few stable isotopes not being considered in the nuclear network as they are not accessible by the s-process (see Section 2). For comparison, the final surface abundance distribution for the $2 M_{\odot}$ model of Cristallo et al. (2009) is shown as a solid gray line. The elements are ordered by increasing atomic number.

(A color version of this figure is available in the online journal.)

Among the light elements, $[\mathrm{C} / \mathrm{Fe}],[\mathrm{F} / \mathrm{Fe}],[\mathrm{Ne} / \mathrm{Fe}]$, and $[\mathrm{Na} / \mathrm{Fe}]$ are enhanced by over 1 dex by the end of the AGB phase. The final $[\mathrm{C} / \mathrm{Fe}]$ ratio is 1.88 as a result of TDU mixing up the products of partial He-burning. The $[\mathrm{F} / \mathrm{Fe}]$ ratio increases from slightly below the solar value at the start of the AGB phase to 2.10 , higher than the enhancement of $[\mathrm{C} / \mathrm{Fe}]$. The $[\mathrm{O} / \mathrm{Fe}]$ value increases marginally as a result of partial He-burning to 0.30 while the abundance of $[\mathrm{N} / \mathrm{Fe}]$ only increases by $0.05 \mathrm{dex}$ during the AGB phase. The final surface abundances of $[\mathrm{Ne} / \mathrm{Fe}]$ and $[\mathrm{Na} / \mathrm{Fe}]$ are enhanced to 1.3 and 1.2 , respectively. The Cristallo et al. (2009) model has a higher enhancement in $[\mathrm{Ne} / \mathrm{Fe}]$ and $[\mathrm{Mg} / \mathrm{Fe}]$ with values up to 1.60 and 1.28 , respectively. For the Fe-peak elements, there are minimal changes in the abundances. Both $\mathrm{Co}$ and $\mathrm{Cu}$ experience an enhancement of 0.2 dex while Sc increases by 0.14 dex and Zn by 0.1 dex.

The surface abundance of the neutron-capture elements only increases after the second TDU episode (Figure 4). The ${ }^{13} \mathrm{C}$ pocket burns during the interpulse period once protons are added at the deepest extent of the first TDU. The newly synthesized neutron-capture elements are then mixed to the surface during the next TDU. The $s$-process abundance for each element asymptotically approaches its final value as each TDU brings more $s$-process enriched material to the surface. By the end of the AGB phase, the ratios of $[\mathrm{Rb} / \mathrm{Fe}]$, $[\mathrm{Zr} / \mathrm{Fe}],[\mathrm{Ba} / \mathrm{Fe}]$, and $[\mathrm{Pb} / \mathrm{Fe}]$ are enhanced by $0.70,1.53,2.02$, and 2.95 dex, respectively. These values follow a characteristic abundance distribution of neutron-capture elements for a lowmetallicity low-mass AGB model where the production of $\mathrm{Pb}$ is favored over the other neutron-capture elements (Busso et al. 2001). The model of Cristallo et al. (2009) produces 1.41, 1.99, and 2.87 , respectively, for $[\mathrm{Zr} / \mathrm{Fe}],[\mathrm{Ba} / \mathrm{Fe}]$, and $[\mathrm{Pb} / \mathrm{Fe}]$ and these values are comparable to those presented here despite a different treatment of the inner border of the convective envelope. The Cristallo et al. (2009) model has a noticeably higher enhancement of $\mathrm{Rb}$ as seen in Figure 4 as a result of neutron densities greater than $10^{12} \mathrm{ncm}^{-3}$ occurring during a TP. The neutron densities in our model peak at less than $10^{11} n \mathrm{~cm}^{-3}$ during a TP (see Figure 5) and have a minimal contribution to the abundance of $\mathrm{Rb}$.
The surface abundance distribution for the neutron-capture elements exhibits three main peaks (around $\mathrm{Sr}, \mathrm{Ba}$, and $\mathrm{Pb}$ as seen in Figure 4) corresponding to the isotopes with a magic number of neutrons $(N=50,82,126)$. The average abundances of the neutron-capture elements at the first two of these points are called light $s(l s)$ and heavy $s(h s)$, respectively. The third peak is given as the abundance of $[\mathrm{Pb} / \mathrm{Fe}]$. As in Cristallo et al. (2011), the $[l s / \mathrm{Fe}]$ abundance is given by

$$
[l s / \mathrm{Fe}]=([\mathrm{Sr} / \mathrm{Fe}]+[\mathrm{Y} / \mathrm{Fe}]+[\mathrm{Zr} / \mathrm{Fe}]) / 3,
$$

and the $[h s / \mathrm{Fe}]$ abundance,

$$
[h s / \mathrm{Fe}]=([\mathrm{Ba} / \mathrm{Fe}]+[\mathrm{La} / \mathrm{Fe}]+[\mathrm{Nd} / \mathrm{Fe}]+[\mathrm{Sm} / \mathrm{Fe}]) / 4 \text {. }
$$

As the neutron exposure increases, the $l s$ elements are produced first, then the $h s$ elements, and finally $\mathrm{Pb}$. For the $2 M_{\odot}$ model, the final surface abundance values of $[l s / \mathrm{Fe}],[h s / \mathrm{Fe}]$, and $[\mathrm{Pb} / \mathrm{Fe}]$ are $1.43,1.95$, and 2.95 , respectively. Combinations of these ratios include $[h s / l s]$ and $[\mathrm{Pb} / h s]$ and these $s$-process indicators are independent of the efficiency of TDU and the mass loss rate for the low-mass models. For the model presented here the final $[h s / l s]$ and $[\mathrm{Pb} / h s]$ values are 0.52 and 1.00 . These values are reached by the sixth TDU episode and remain constant until the end of the AGB phase.

The abundance distribution of the neutron-capture elements is predominately controlled by the neutron density, along with the neutron exposure. In Figure 5, we plot, against time, the temperature of the He-burning shell and the maximum neutron density reached for each of the TPs. Notably, the first interpulse period with a ${ }^{13} \mathrm{C}$ pocket has a neutron density around $10^{7} \mathrm{n} \mathrm{cm}^{-3}$. During this interpulse, not all of the ${ }^{13} \mathrm{C}$ is burned radiatively and is later engulfed by the subsequent TP, resulting in convective ${ }^{13} \mathrm{C}$ burning. This condition is described by Lugaro et al. (2012) as Case 3. For the remaining ${ }^{13} \mathrm{C}$ pockets, all the ${ }^{13} \mathrm{C}$ is burned radiatively before the subsequent $\mathrm{TP}$ in accordance with Case 2 as described by Lugaro et al. (2012). A peak in neutron density occurs at each TP where there is a marginal activation of the ${ }^{22} \mathrm{Ne}$ neutron source (Gallino et al. 1998). The production of the neutron-capture elements from the ${ }^{22} \mathrm{Ne}$ source 

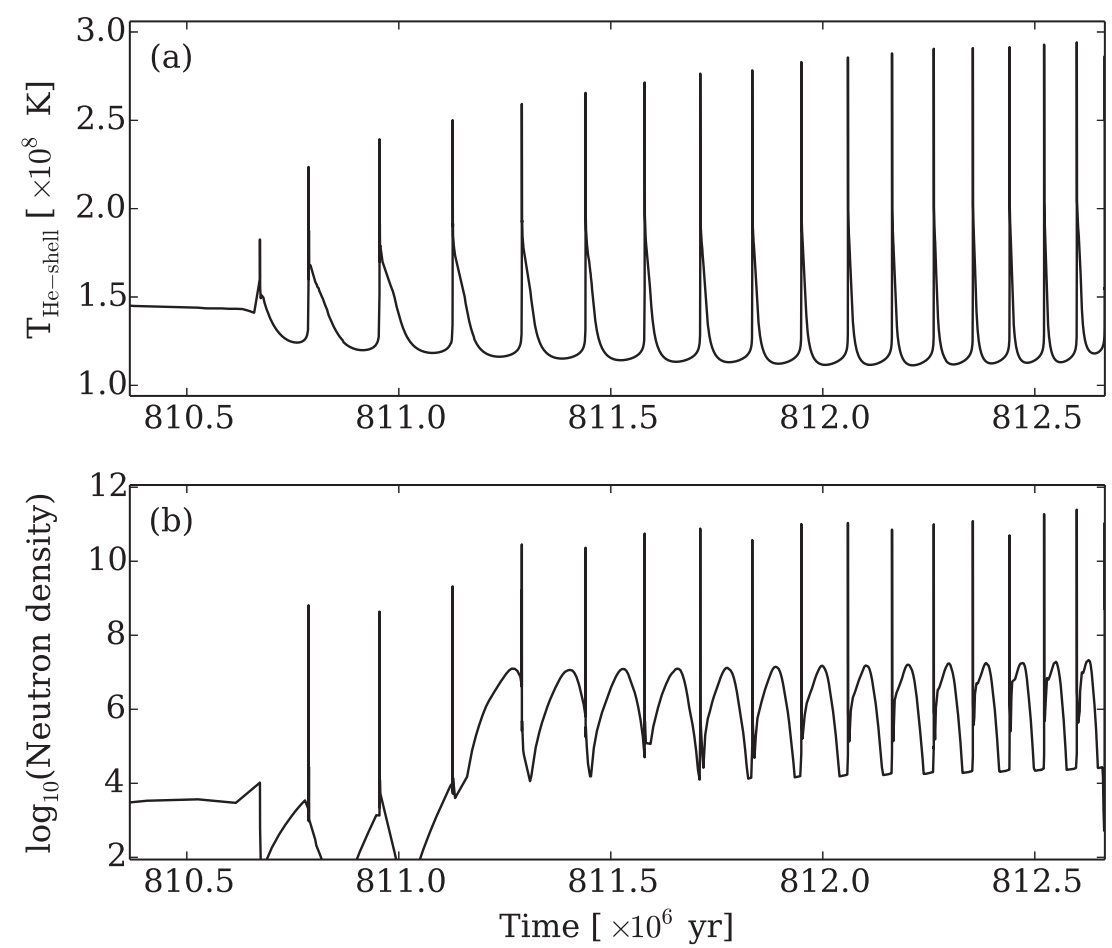

Figure 5. (a) Temperature at the top of the He-shell and (b) the maximum neutron density as a function of time from the start of the AGB phase for the $2 M_{\odot}$ model.
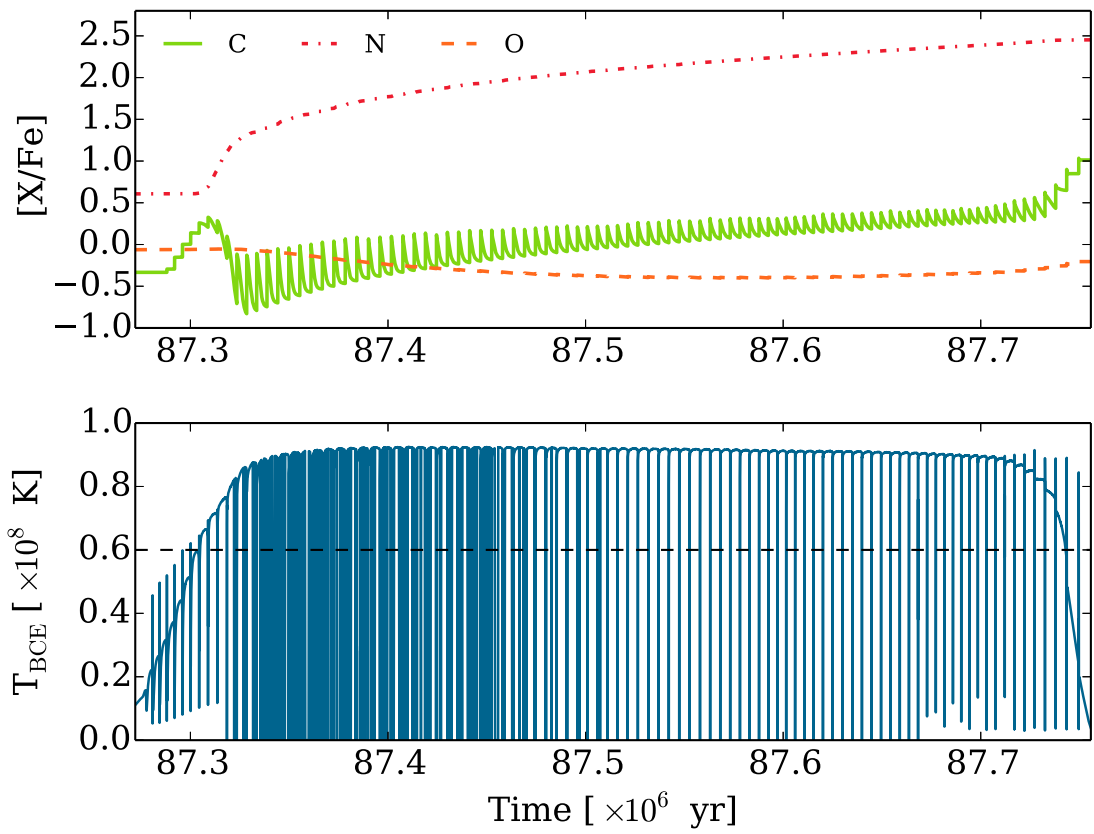

Figure 6. Evolution of $[\mathrm{C} / \mathrm{Fe}],[\mathrm{N} / \mathrm{Fe}]$, and $[\mathrm{O} / \mathrm{Fe}]$ (top) and the temperature at the base of the convective envelope (bottom) with respect to time from the start of the AGB phase for the $5 M_{\odot}$ model. The dashed line illustrates the temperature above which HBB is efficiently activated.

(A color version of this figure is available in the online journal.)

is negligible compared to those produced from the ${ }^{13} \mathrm{C}$ neutron source but elements produced via branching points such as $\mathrm{Rb}$ can be affected by this neutron flux.

\subsection{The $5 M_{\odot}$ Model}

We examine the $5 M_{\odot}$ model as a representative case of an intermediate-mass AGB model. In contrast to a low-mass AGB model, the intermediate-mass models experience more efficient HBB and the ${ }^{22} \mathrm{Ne}$ neutron source is more efficiently activated.
We do not add a PMZ for the reasons presented in detail by García-Hernández et al. (2013).

Figure 6 illustrates (1) the effect of $\mathrm{HBB}$ on the $\mathrm{C}, \mathrm{N}$, and $\mathrm{O}$ surface abundance relative to $\mathrm{Fe}$ and (2) the temperature at the base of the convective envelope. Initially, when the temperature at the base of the convective envelope has not reached the value required for $\mathrm{CNO}$ cycling, the ratio of $[\mathrm{C} / \mathrm{Fe}]$ increases due to TDU while $[\mathrm{N} / \mathrm{Fe}]$ remains constant. When temperatures at the base of the convective envelope reach approximately $60 \times 10^{6} \mathrm{~K}$ the $\mathrm{CNO}$ cycle is activated with $\mathrm{C}$ and $\mathrm{O}$ being converted to $\mathrm{N}$. 


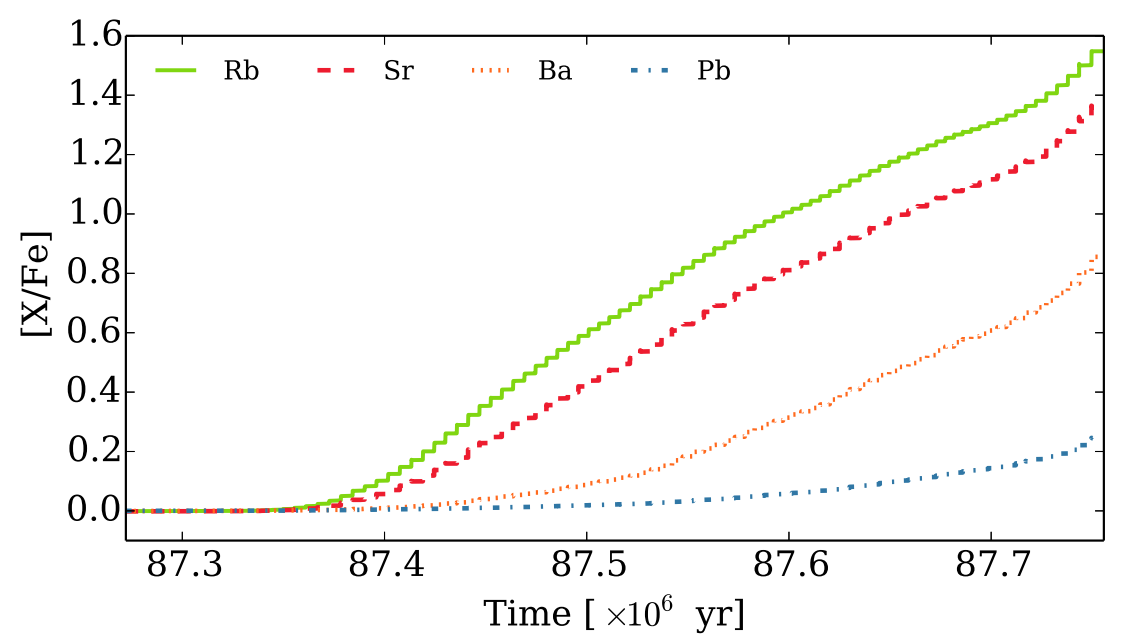

Figure 7. Surface abundance of four neutron-capture elements $\left(\mathrm{Rb}, \mathrm{Sr}, \mathrm{Ba}\right.$, and $\mathrm{Pb}$ ) as a function of time from the start of the AGB phase for the $5 M_{\odot}$ model. (A color version of this figure is available in the online journal.)

This destruction of $\mathrm{C}$ and $\mathrm{O}$, along with the competing effect of TDU, causes the evolution of the $\mathrm{C} / \mathrm{O}$ ratio to fluctuate about unity. From a certain point onward, the model remains carbon rich. Eventually $\mathrm{HBB}$ is extinguished and $[\mathrm{C} / \mathrm{Fe}]$ and $[\mathrm{O} / \mathrm{Fe}]$ once again increases for the last few TDU episodes.

This fluctuating behavior in the $\mathrm{C} / \mathrm{O}$ ratio is also a feature in the $5 M_{\odot}, Z=0.001$ model of Marigo et al. (2013). Marigo et al. (2013) also noted that the last few TPs do not experience $\mathrm{HBB}$ and the surface $\mathrm{C} / \mathrm{O}$ ratio increases significantly to a final value of $\sim 10$. This value is comparable to the final $\mathrm{C} / \mathrm{O}$ ratio of $\sim 9$ for the $5 M_{\odot}$ model presented here. One difference, however, is that prior to HBB the Marigo et al. (2013) model does not exhibit an increase in the $\mathrm{C} / \mathrm{O}$ ratio in contrast to our model in which this ratio increases above unity before the onset of $\mathrm{HBB}$ (Figure 6).

The efficiency of TDU in the $5 M_{\odot}$ model increases with each TP until it reaches a plateau of $\lambda \approx 0.95$ and the amount of material brought to the surface gradually increases until it reaches a value of approximately $2.5 \times 10^{-3} M_{\odot}$. Despite the high efficiency of TDU, the amount of material mixed to the surface through each TDU is lower for the $5 M_{\odot}$ model compared to the low-mass model of $2 M_{\odot}$. This is a result of the He-intershell region having approximately 10 times less mass. However, the higher number of TDU episodes (93 compared to 14) means that the overall amount of the material being brought to the surface during the AGB phase is larger, $0.194 M_{\odot}$ compared to $0.095 M_{\odot}$ for the $2 M_{\odot}$ model (see Table 1 ).

Figure 7 presents the evolution of $\mathrm{Rb}, \mathrm{Sr}, \mathrm{Ba}$, and $\mathrm{Pb}$ relative to Fe. These elements are representative of the three $s$-process peaks. $\mathrm{Rb}$ exhibits the greatest enhancement and $\mathrm{Pb}$ the least and this situation is opposite to what the low-mass models display. The intermediate-mass models do not attain the neutron exposure required to produce elements such as $\mathrm{Ba}$ and $\mathrm{Pb}$ to the level produced by the low-mass models. However, the models are able to produce the high neutron densities required to bypass unstable isotopes (e.g., ${ }^{85} \mathrm{Kr}$ ) at branching points resulting in a higher abundance of $\mathrm{Rb}$ (see van Raai et al. 2012). The final $[\mathrm{Rb} / \mathrm{Fe}]$ surface abundance is around 1.6 whereas $[\mathrm{Pb} / \mathrm{Fe}]$ only increases by approximately 0.2 dex.

Figure 8(a) illustrates the maximum neutron density reached for each TP as a function of time. The peak neutron density is approximately $10^{13} n \mathrm{~cm}^{-3}$. The neutron density stays above $10^{12} n \mathrm{~cm}^{-3}$ for approximately 30 days within each TP. As the temperature of the He-burning shell decreases, the neutron density also decreases. During the interpulse, the neutron density remains constant just below $10^{6} n \mathrm{~cm}^{-3}$ due to the release of neutrons during radiative burning in the He-shell. However, this neutron flux is not high enough to activate $s$-process nucleosynthesis.

The surface $[h s / l s]$ and $[\mathrm{Pb} / h s]$ ratios from the beginning of the AGB phase are presented in Figures 8(b) and (c). The behavior of the $s$-process indicators for the $5 M_{\odot}$ model (and all the intermediate-mass models) differs from the low-mass models due to the different neutron source. For the $5 M_{\odot}$ model, the ratios of $[l s / \mathrm{Fe}],[h s / \mathrm{Fe}],[h s / l s]$, and $[\mathrm{Pb} / h s]$ remain constant during the AGB phase until the neutron density reaches above $10^{12} n \mathrm{~cm}^{-3}$. Once the neutron density exceeds this value the ratios of $[l s / \mathrm{Fe}]$ and $[h s / \mathrm{Fe}]$ increase while $[h s / l s]$ and $[\mathrm{Pb} / h s]$ decrease. Unlike the low-mass models, the values of $[h s / l s]$ and $[\mathrm{Pb} / h s]$ never reach an equilibrium value.

\subsection{Comparison with Different Metallicities}

We briefly compare our 2 and $5 M_{\odot}$ models of $[\mathrm{Fe} / \mathrm{H}]=$ -1.2 to the models of $[\mathrm{Fe} / \mathrm{H}]=-2.3$ presented in Lugaro et al. (2012) and the models of $[\mathrm{Fe} / \mathrm{H}]=-1.7$ presented in Straniero et al. (2014). As the models of Straniero et al. (2014) are $\alpha$ enhanced we only examine the neutron-capture elements ( $\mathrm{Ga}$ to $\mathrm{Bi}$ ) which are unaffected by the initial abundance of $\alpha$ elements.

Figure 9 illustrates the final neutron-capture surface abundances in $[\mathrm{X} / \mathrm{Fe}]$ for each of the models. Both the models calculated here and in Lugaro et al. (2012) use the same evolutionary and post-processing codes, while the models of Cristallo et al. (2011) and Straniero et al. (2014) use the FUNS code. The dip in abundance at $\mathrm{Nb}$ for the Cristallo et al. (2011) and Straniero et al. (2014) models is due to the unstable isotope ${ }^{93} \mathrm{Zr}$ $\left(\tau_{1 / 2}=1.53 \times 10^{6} \mathrm{yr}\right)$ not being decayed to the stable isotope ${ }^{93} \mathrm{Nb}$. We include the $2 M_{\odot}, Z=0.001$ model from Cristallo et al. (2011) in Figure 9 for completeness.

Table 5 presents a number of abundance ratios including the $s$-process indicators. The models of Straniero et al. (2014) have a $[\mathrm{Fe} / \mathrm{H}]$ value in between the other models and we would expect them to show intermediate abundance values. However, the $2 M_{\odot}$ model appears to have essentially the same abundance pattern of the other models but with overall lower abundances. This is possibly the result of a lower amount of mass dredged up $\left(0.075 M_{\odot}\right)$ for their model, probably a consequence of the 

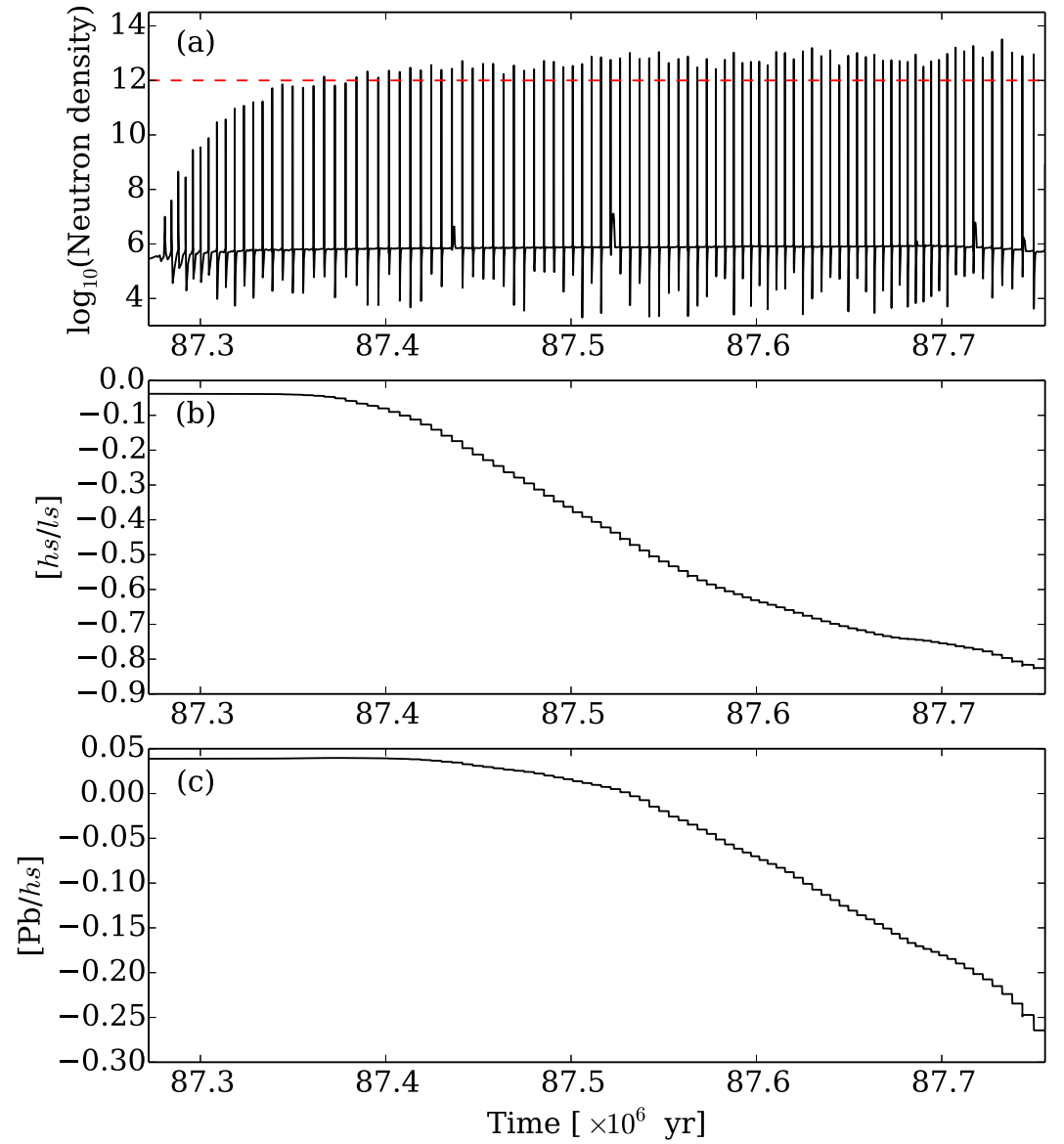

Figure 8. (a) Maximum neutron density (the red dashed line shows the density where the ${ }^{22} \mathrm{Ne}$ neutron source is efficient), (b) the surface $[h s / l s]$ ratio, and (c) the surface $[\mathrm{Pb} / h s]$ ratio as a function of time for the $5 M_{\odot}$ model from the beginning of the AGB phase.

(A color version of this figure is available in the online journal.)

Table 5

Final Surface Abundances of the 2 and $5 M_{\odot}$ Models for a Number of Neutron-capture Elemental Ratios for Each Metallicity

\begin{tabular}{|c|c|c|c|c|c|c|}
\hline & \multicolumn{2}{|c|}{$\begin{array}{c}\mathrm{L} 12 \\
{[\mathrm{Fe} / \mathrm{H}]=-2.3}\end{array}$} & \multicolumn{2}{|c|}{$\begin{array}{c}\mathrm{S} 14 \\
{[\mathrm{Fe} / \mathrm{H}]=-1.7}\end{array}$} & \multicolumn{2}{|c|}{$\begin{array}{c}\mathrm{F} 14 \\
{[\mathrm{Fe} / \mathrm{H}]=-1.2}\end{array}$} \\
\hline & $2 M_{\odot}$ & $5 M_{\odot}$ & $2 M_{\odot}$ & $5 M_{\odot}$ & $2 M_{\odot}$ & $5 M_{\odot}$ \\
\hline$[\mathrm{Rb} / \mathrm{Fe}]$ & 1.47 & 1.86 & 0.60 & 1.37 & 0.70 & 1.55 \\
\hline$[\mathrm{Zr} / \mathrm{Fe}]$ & 1.96 & 1.74 & 0.94 & 0.98 & 1.53 & 1.32 \\
\hline$[\mathrm{Rb} / \mathrm{Zr}]$ & -0.49 & 0.12 & -0.34 & 0.39 & -0.83 & 0.24 \\
\hline$[l s / \mathrm{Fe}]$ & 1.87 & 1.72 & 0.89 & 1.00 & 1.43 & 1.34 \\
\hline$[h s / \mathrm{Fe}]$ & 2.36 & 1.07 & 1.40 & 0.63 & 1.95 & 0.52 \\
\hline$[\mathrm{Pb} / \mathrm{Fe}]$ & 3.24 & 0.71 & 2.77 & 1.43 & 2.95 & 0.25 \\
\hline$[h s / l s]$ & 0.49 & -0.66 & 0.51 & -0.37 & 0.52 & -0.82 \\
\hline$[\mathrm{Pb} / h s]$ & 0.88 & -0.36 & 1.37 & 0.80 & 1.01 & -0.27 \\
\hline
\end{tabular}

Note. L12 (Lugaro et al. 2012), S14 (Straniero et al. 2014), F14 (models presented here).

$\alpha$ enhancement employed in these models. The $5 M_{\odot}$ model of Straniero et al. (2014) has a higher surface abundance of $\mathrm{Pb}$ compared to the $5 M_{\odot}$ model presented here and in Lugaro et al. (2012). This is due to the contribution of a small ${ }^{13} \mathrm{C}$ pocket activated after each TDU in the model of Straniero et al. (2014), which is not included in the other $5 M_{\odot}$ models.

\section{ABUNDANCE AND STELLAR YIELD RESULTS}

In this section, we present final surface abundances and elemental stellar yields for each of the calculated models. We calculate the net stellar yield $M_{i}$ (in $M_{\odot}$ ) to be

$$
M_{i}=\int_{0}^{\tau}\left[X(i)-X_{0}(i)\right] \frac{d M}{d t} d t,
$$

where $d M / d t$ is the current mass loss rate in $M_{\odot} \mathrm{yr}^{-1}, X(i)$ and $X_{0}(i)$ are the current and initial mass fraction of species $i$, and $\tau$ is the total lifetime of the stellar model (Karakas 2010). For a negative net yield, the species is destroyed whereas a positive net yield indicates that the species is produced. Tables 6 and 7 present net yields of select elements for each model. We have made available online tables presenting yields for isotopes up to the Fe group and all the elements. We provide for each model: the species $i$; the atomic number; the net stellar yield as defined in Equation (4); the amount of the species $i$ in the wind lost from the star, which is always positive; and the total mass expelled during the stellar lifetime multiplied by the initial mass fraction, $M_{0}(i)$. We also include the average mass fraction of $i$ in the wind $\langle X(i)\rangle$, the initial mass fraction $X_{0}(i)$, and the production factor $f$ defined as $\log _{10}\left[\langle X(i)\rangle / X_{0}(i)\right]$. Tables 8 and 9 show a portion of the yields table for the isotopes and elements and are published in their entirety in the online journal. In addition, Tables 10 and 11 show a portion of the final surface abundances table for the isotopes and elements and are published in their entirety in the online journal.

\subsection{The Light Elements}

In this section, we present final surface abundances and yields for the light elements up to the Fe group. We discuss $\mathrm{He}, \mathrm{C}, \mathrm{N}$, 

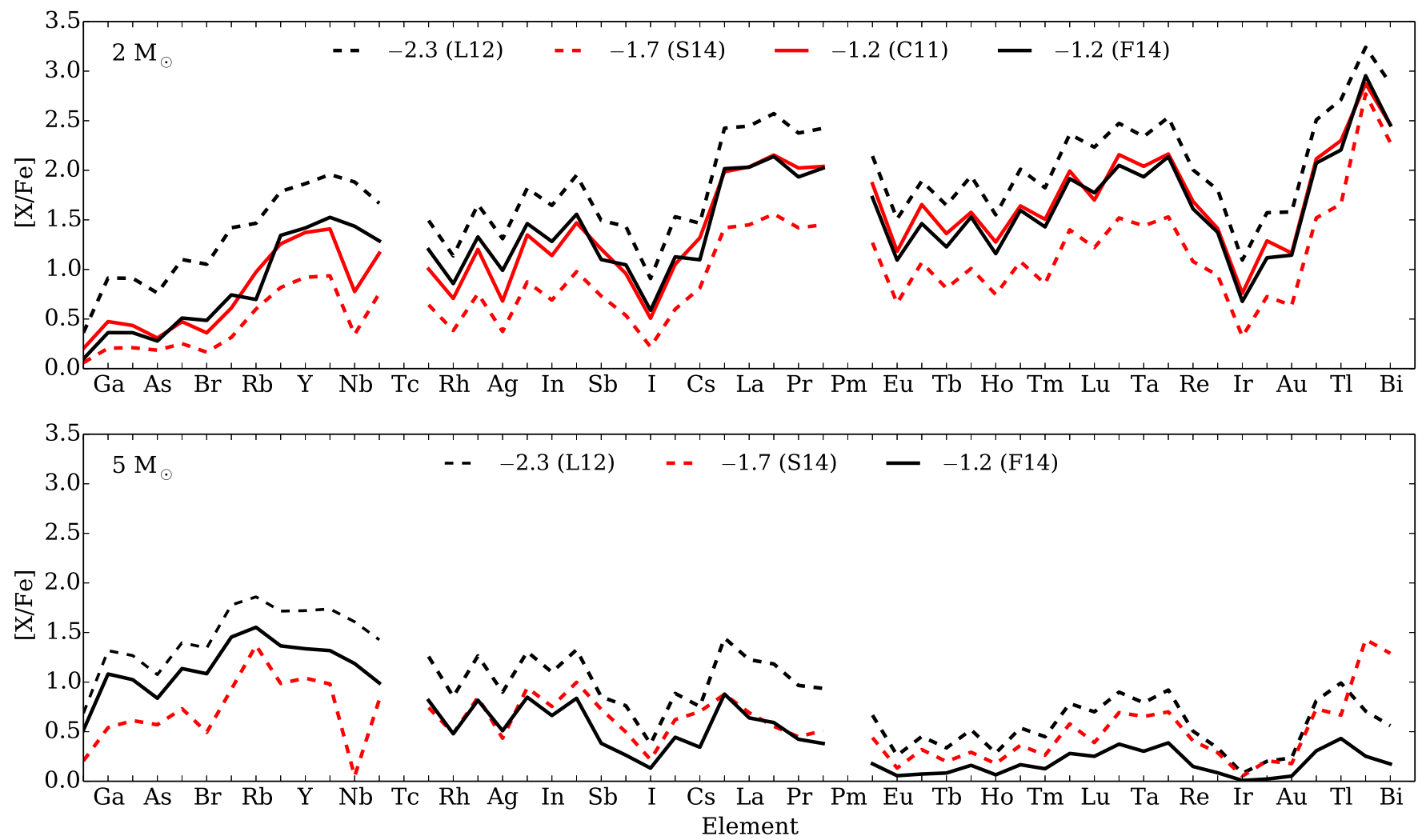

Figure 9. Final surface abundance ratios of neutron-capture elements for three different $[\mathrm{Fe} / \mathrm{H}]$ ratios for the $2 M_{\odot}$ models (top) and $5 M_{\odot}$ models (bottom). Legend is as follows: C11 (Cristallo et al. 2011), L12 (Lugaro et al. 2012), S14 (Straniero et al. 2014), F14 (models presented here). The elements are ordered by increasing atomic number.

(A color version of this figure is available in the online journal.)

Table 6

Net Stellar Yield Results of Selected Light and Neutron-capture Elements for Each Low-mass Model

\begin{tabular}{lclllllll}
\hline \hline El & $1 M_{\odot}$ & $1.25 M_{\odot}$ & $1.5 M_{\odot}$ & $2 M_{\odot}$ & $2.25 M_{\odot}$ & $2.5 M_{\odot}$ & $2.75 M_{\odot}$ & $3 M_{\odot}$ \\
\hline $\mathrm{He}$ & $8.56(-3)$ & $2.08(-2)$ & $3.16(-2)$ & $6.76(-2)$ & $8.48(-2)$ & $8.22(-2)$ & $8.25(-2)$ & $7.63(-2)$ \\
$\mathrm{C}$ & $1.92(-4)$ & $1.57(-3)$ & $4.94(-3)$ & $1.65(-2)$ & $2.22(-2)$ & $2.34(-2)$ & $2.53(-2)$ & $2.44(-2)$ \\
$\mathrm{N}$ & $1.95(-5)$ & $5.11(-5)$ & $8.09(-5)$ & $1.49(-4)$ & $1.72(-4)$ & $1.69(-4)$ & $1.85(-4)$ & $2.34(-4)$ \\
$\mathrm{O}$ & $3.59(-6)$ & $6.82(-5)$ & $2.16(-4)$ & $5.34(-4)$ & $6.32(-4)$ & $6.34(-4)$ & $5.17(-4)$ & $4.71(-4)$ \\
$\mathrm{F}$ & $5.63(-9)$ & $1.86(-7)$ & $7.27(-7)$ & $3.77(-6)$ & $6.40(-6)$ & $7.52(-6)$ & $6.56(-6)$ & $4.00(-6)$ \\
$\mathrm{Ne}$ & $5.05(-6)$ & $1.41(-4)$ & $5.56(-4)$ & $2.45(-3)$ & $3.64(-3)$ & $4.03(-3)$ & $2.99(-3)$ & $2.01(-3)$ \\
$\mathrm{Na}$ & $1.37(-7)$ & $1.49(-6)$ & $6.52(-6)$ & $3.65(-5)$ & $5.24(-5)$ & $4.29(-5)$ & $2.64(-5)$ & $2.09(-5)$ \\
$\mathrm{Mg}$ & $-2.17(-8)$ & $1.33(-6)$ & $6.49(-6)$ & $3.86(-5)$ & $6.92(-5)$ & $1.18(-4)$ & $1.44(-4)$ & $1.77(-4)$ \\
$\mathrm{Al}$ & $6.74(-8)$ & $1.80(-7)$ & $3.75(-7)$ & $1.36(-6)$ & $2.68(-6)$ & $6.14(-6)$ & $7.46(-6)$ & $6.56(-6)$ \\
$\mathrm{Si}$ & $1.22(-8)$ & $7.36(-8)$ & $1.82(-7)$ & $5.84(-7)$ & $1.16(-6)$ & $3.46(-6)$ & $5.99(-6)$ & $9.09(-6)$ \\
$\mathrm{Rb}$ & $2.69(-11)$ & $5.76(-10)$ & $2.51(-9)$ & $6.89(-9)$ & $1.40(-8)$ & $3.41(-8)$ & $2.62(-8)$ & $2.41(-8)$ \\
$\mathrm{Sr}$ & $5.51(-10)$ & $6.55(-9)$ & $2.68(-8)$ & $8.65(-8)$ & $1.25(-7)$ & $1.37(-7)$ & $8.33(-8)$ & $5.44(-8)$ \\
$\mathrm{Y}$ & $1.15(-10)$ & $1.65(-9)$ & $6.29(-9)$ & $2.24(-8)$ & $3.36(-8)$ & $4.02(-8)$ & $2.52(-8)$ & $1.55(-8)$ \\
$\mathrm{Zr}$ & $2.33(-10)$ & $4.64(-9)$ & $1.65(-8)$ & $6.23(-8)$ & $9.57(-8)$ & $1.19(-7)$ & $8.14(-8)$ & $5.41(-8)$ \\
$\mathrm{Ba}$ & $1.35(-10)$ & $9.32(-9)$ & $3.09(-8)$ & $1.32(-7)$ & $2.02(-7)$ & $2.23(-7)$ & $1.34(-7)$ & $8.69(-8)$ \\
$\mathrm{La}$ & $1.44(-11)$ & $9.23(-10)$ & $3.14(-9)$ & $1.35(-8)$ & $2.07(-8)$ & $2.28(-8)$ & $1.30(-8)$ & $7.81(-9)$ \\
$\mathrm{Ce}$ & $5.85(-11)$ & $2.87(-9)$ & $1.04(-8)$ & $4.49(-8)$ & $7.03(-8)$ & $8.00(-8)$ & $4.80(-8)$ & $2.94(-8)$ \\
$\mathrm{Pb}$ & $4.69(-9)$ & $1.19(-7)$ & $4.14(-7)$ & $1.27(-6)$ & $1.65(-6)$ & $1.64(-6)$ & $1.27(-6)$ & $9.11(-7)$ \\
\hline
\end{tabular}

Note. Yields are in solar masses and are expressed in the form $n(m)=n \times 10^{m}$.

$\mathrm{O}, \mathrm{F}, \mathrm{Ne}, \mathrm{Na}, \mathrm{Mg}$, and $\mathrm{Al}$ in detail. These elements are well known to be produced or destroyed in AGB stars (Busso et al. 1999). In Figure 10 we present the final surface abundances of select light elements (in [X/Fe]) for each of the models. Table 12 presents the final surface abundances for the ${ }^{4} \mathrm{He}$ mass fraction, $\mathrm{C} / \mathrm{O}$ ratio, ${ }^{12} \mathrm{C} /{ }^{13} \mathrm{C}$ ratio, and $[\mathrm{X} / \mathrm{Fe}]$ for the selected light elements.

\subsection{1. $\mathrm{He}, \mathrm{C}, \mathrm{N}, \mathrm{O}$, and $\mathrm{F}$}

As presented in Table 12, the final ${ }^{4} \mathrm{He}$ surface abundance for the low-mass models reaches a peak value of 0.31 for the $2.25 M_{\odot}$ model. This is a result of the $2.25 M_{\odot}$ model experiencing the deepest extent of FDU and efficient TDU. The $2.25 M_{\odot}$ model also has the highest He yield of the 
Table 7

Net Stellar Yield Results of Selected Light and Neutron-capture Elements for Each Intermediate-mass Model

\begin{tabular}{|c|c|c|c|c|c|c|c|c|}
\hline El. & $3.25 M_{\odot}$ & $3.5 M_{\odot}$ & $4 M_{\odot}$ & $4.5 M_{\odot}$ & $5 M_{\odot}$ & $5.5 M_{\odot}$ & $6 M_{\odot}$ & $7 M_{\odot}$ \\
\hline $\mathrm{He}$ & $6.89(-2)$ & $1.01(-1)$ & $2.66(-1)$ & $3.57(-1)$ & $4.39(-1)$ & $5.01(-1)$ & $5.48(-1)$ & $6.06(-1)$ \\
\hline $\mathrm{C}$ & $1.95(-2)$ & $1.56(-2)$ & $2.39(-3)$ & $3.04(-3)$ & $2.63(-3)$ & $1.80(-3)$ & $1.74(-3)$ & $2.56(-4)$ \\
\hline $\mathrm{N}$ & $1.24(-3)$ & $8.53(-3)$ & $5.94(-2)$ & $5.61(-2)$ & $5.22(-2)$ & $4.33(-2)$ & $3.29(-2)$ & $1.26(-2)$ \\
\hline $\mathrm{O}$ & $2.19(-4)$ & $2.99(-4)$ & $3.09(-4)$ & $-2.62(-4)$ & $-8.28(-4)$ & $-1.24(-3)$ & $-1.61(-3)$ & $-2.20(-3)$ \\
\hline $\mathrm{F}$ & $1.27(-6)$ & $1.28(-6)$ & $1.45(-7)$ & $7.97(-8)$ & $-4.54(-9)$ & $-6.43(-8)$ & $-1.02(-7)$ & $-1.41(-7)$ \\
\hline $\mathrm{Ne}$ & $6.58(-4)$ & $6.77(-4)$ & $2.93(-3)$ & $2.21(-3)$ & $1.64(-3)$ & $9.32(-4)$ & $4.84(-4)$ & $5.81(-5)$ \\
\hline $\mathrm{Na}$ & $1.04(-5)$ & $1.26(-5)$ & $7.57(-5)$ & $5.94(-5)$ & $3.86(-5)$ & $1.64(-5)$ & $3.65(-6)$ & $-8.63(-6)$ \\
\hline $\mathrm{Mg}$ & $9.45(-5)$ & $1.02(-4)$ & $7.53(-4)$ & $6.56(-4)$ & $4.47(-4)$ & $2.75(-4)$ & $1.39(-4)$ & $-6.84(-5)$ \\
\hline $\mathrm{Al}$ & $3.14(-6)$ & $3.15(-6)$ & $4.01(-5)$ & $4.88(-5)$ & $5.86(-5)$ & $6.89(-5)$ & $8.55(-5)$ & $7.00(-5)$ \\
\hline $\mathrm{Si}$ & $6.73(-6)$ & $7.63(-6)$ & $3.37(-5)$ & $3.19(-5)$ & $2.68(-5)$ & $2.22(-5)$ & $2.57(-5)$ & $7.73(-5)$ \\
\hline $\mathrm{Rb}$ & $1.48(-8)$ & $1.72(-8)$ & $1.53(-7)$ & $1.64(-7)$ & $1.38(-7)$ & $1.12(-7)$ & $8.50(-8)$ & $2.41(-8)$ \\
\hline $\mathrm{Sr}$ & $1.72(-8)$ & $2.05(-8)$ & $2.20(-7)$ & $2.38(-7)$ & $2.04(-7)$ & $1.67(-7)$ & $1.22(-7)$ & $3.06(-8)$ \\
\hline $\mathrm{Y}$ & $2.96(-9)$ & $3.56(-9)$ & $4.40(-8)$ & $4.85(-8)$ & $4.05(-8)$ & $3.38(-8)$ & $2.40(-8)$ & $5.79(-9)$ \\
\hline $\mathrm{Zr}$ & $5.23(-9)$ & $6.28(-9)$ & $8.99(-8)$ & $1.01(-7)$ & $8.27(-8)$ & $6.93(-8)$ & $4.78(-8)$ & $1.09(-8)$ \\
\hline $\mathrm{Ba}$ & $7.75(-10)$ & $9.17(-10)$ & $2.01(-8)$ & $2.28(-8)$ & $1.78(-8)$ & $1.50(-8)$ & $9.15(-9)$ & $1.77(-9)$ \\
\hline $\mathrm{La}$ & $3.26(-11)$ & $4.03(-11)$ & $1.06(-9)$ & $1.17(-9)$ & $8.93(-10)$ & $7.55(-10)$ & $4.46(-10)$ & $8.11(-11)$ \\
\hline $\mathrm{Ce}$ & $7.83(-11)$ & $9.41(-11)$ & $2.41(-9)$ & $2.71(-9)$ & $2.01(-9)$ & $1.68(-9)$ & $9.64(-10)$ & $1.75(-10)$ \\
\hline $\mathrm{Pb}$ & $1.60(-10)$ & $1.79(-10)$ & $2.51(-9)$ & $2.90(-9)$ & $2.27(-9)$ & $1.82(-9)$ & $9.65(-10)$ & $1.72(-10)$ \\
\hline
\end{tabular}

Note. Yields are in solar masses and are expressed in the form $n(m)=n \times 10^{m}$.

Table 8

An Example of the Online Data Table Providing Isotopic Yields

\begin{tabular}{|c|c|c|c|c|c|c|c|c|}
\hline Mass $^{a}$ & Species $^{b}$ & $A^{\mathrm{c}}$ & $M_{i}^{\mathrm{d}}$ & $M_{\mathrm{lost}}(i)^{\mathrm{e}}$ & $M_{0}(i)^{\mathrm{f}}$ & $\langle X(i)\rangle^{\mathrm{g}}$ & $X_{0}(i)^{\mathrm{h}}$ & $f^{\mathrm{i}}$ \\
\hline 1.0 & $n$ & 1 & $0.00 \mathrm{e}+0$ & $0.00 \mathrm{e}+0$ & $0.00 \mathrm{e}+0$ & $0.00 \mathrm{e}+0$ & $0.00 \mathrm{e}+0$ & $0.00 \mathrm{e}+0$ \\
\hline 1.0 & $p$ & 1 & $-8.78 \mathrm{e}-3$ & $2.41 \mathrm{e}-1$ & $2.49 \mathrm{e}-1$ & $7.23 e-1$ & $7.49 \mathrm{e}-1$ & $-1.56 \mathrm{e}-2$ \\
\hline 1.0 & $d$ & 2 & $-6.02 \mathrm{e}-7$ & $4.82 \mathrm{e}-7$ & $1.08 \mathrm{e}-6$ & $1.45 e-6$ & $3.26 \mathrm{e}-6$ & $-3.52 \mathrm{e}-1$ \\
\hline 1.0 & ${ }^{3} \mathrm{He}$ & 3 & $8.90 \mathrm{e}-5$ & $9.59 \mathrm{e}-5$ & $6.89 e-6$ & $2.88 \mathrm{e}-4$ & $2.07 \mathrm{e}-5$ & $1.14 \mathrm{e}+0$ \\
\hline 1.0 & ${ }^{4} \mathrm{He}$ & 4 & $8.47 e-3$ & $9.17 \mathrm{e}-2$ & $8.32 \mathrm{e}-2$ & $2.75 \mathrm{e}-1$ & $2.50 \mathrm{e}-1$ & $4.21 \mathrm{e}-2$ \\
\hline 1.0 & ${ }^{7} \mathrm{Li}$ & 7 & $9.56 \mathrm{e}-11$ & $2.87 \mathrm{e}-10$ & $1.91 \mathrm{e}-10$ & $8.63 e-10$ & $5.75 \mathrm{e}-10$ & $1.76 \mathrm{e}-1$ \\
\hline 1.0 & ${ }^{7} \mathrm{Be}$ & 7 & $7.94 \mathrm{e}-12$ & $7.94 \mathrm{e}-12$ & $0.00 \mathrm{e}+0$ & $2.38 \mathrm{e}-11$ & $0.00 \mathrm{e}+0$ & $0.00 \mathrm{e}+0$ \\
\hline 1.0 & ${ }^{8} \mathrm{~B}$ & 8 & $9.20 \mathrm{e}-26$ & $9.20 \mathrm{e}-26$ & $0.00 \mathrm{e}+0$ & $2.76 e-25$ & $0.00 \mathrm{e}+0$ & $0.00 \mathrm{e}+0$ \\
\hline 1.0 & ${ }^{12} \mathrm{C}$ & 12 & $1.90 \mathrm{e}-4$ & $2.47 \mathrm{e}-4$ & $5.70 \mathrm{e}-5$ & $7.43 e-4$ & $1.71 \mathrm{e}-4$ & $6.38 \mathrm{e}-1$ \\
\hline 1.0 & ${ }^{13} \mathrm{C}$ & 13 & $1.32 \mathrm{e}-6$ & $2.01 \mathrm{e}-6$ & $6.90 \mathrm{e}-7$ & $6.04 e-6$ & $2.07 \mathrm{e}-6$ & $4.65 \mathrm{e}-1$ \\
\hline 1.0 & ${ }^{14} \mathrm{C}$ & 14 & $1.39 \mathrm{e}-9$ & $1.39 \mathrm{e}-9$ & $0.00 \mathrm{e}+0$ & $4.16 e-9$ & $0.00 \mathrm{e}+0$ & $0.00 \mathrm{e}+0$ \\
\hline 1.0 & ${ }^{13} \mathrm{~N}$ & 13 & $0.00 \mathrm{e}+0$ & $0.00 \mathrm{e}+0$ & $0.00 \mathrm{e}+0$ & $0.00 \mathrm{e}+0$ & $0.00 \mathrm{e}+0$ & $0.00 \mathrm{e}+0$ \\
\hline 1.0 & ${ }^{14} \mathrm{~N}$ & 14 & $1.95 e-5$ & $3.64 \mathrm{e}-5$ & $1.68 \mathrm{e}-5$ & $1.09 \mathrm{e}-4$ & $5.06 e-5$ & $3.35 \mathrm{e}-1$ \\
\hline 1.0 & ${ }^{15} \mathrm{~N}$ & 15 & $-1.41 \mathrm{e}-8$ & $2.63 e-8$ & $4.03 e-8$ & $7.89 \mathrm{e}-8$ & $1.21 \mathrm{e}-7$ & $-1.86 \mathrm{e}-1$ \\
\hline
\end{tabular}

Notes. Yields of isotopes up to and including ${ }^{70} \mathrm{Zn}$ are available online for all models.

a Initial stellar mass.

b Species $i$.

c Mass number.

${ }^{\mathrm{d}}$ Net stellar yield as defined in Equation (4).

e Amount of the species $i$ in the wind lost from the star.

$\mathrm{f}$ Total mass expelled during the stellar lifetime multiplied by the initial mass fraction.

g Average mass fraction of species $i$ in the wind.

${ }^{\mathrm{h}}$ Initial mass fraction of species $i$.

${ }^{\mathrm{i}}$ Production factor $f$ defined as $\log _{10}\left[\langle X(i)\rangle / X_{0}(i)\right]$.

(This table is available in its entirety in a machine-readable form in the online journal. A portion is shown here for guidance regarding its form and content.)

low-mass models with a value of $8.48 \times 10^{-2} M_{\odot}$ (see Table 6). The ${ }^{4} \mathrm{He}$ abundance reaches a maximum of 0.36 for the $5,5.5$, 6 , and $7 M_{\odot}$ models and the yield increases with increasing initial mass, with a maximum He yield of $6.06 \times 10^{-1} M_{\odot}$ for the $7 M_{\odot}$ model.

The highest final surface abundance of $[\mathrm{C} / \mathrm{Fe}]$ occurs for the $2.25 M_{\odot}$ model with a value of 1.94 . The $7 M_{\odot}$ has the lowest final abundance due to very efficient HBB and a low $M_{\text {TDU }}$ value (see Table 1). The $\mathrm{C}$ yield increases with increasing initial mass for the models up to $2.75 M_{\odot}$. This increase in the yield follows the increase in the value of $M_{\mathrm{TDU}}$ where more $\mathrm{C}$ is mixed to the surface. The highest yield of $\mathrm{C}$ occurs for the $2.75 M_{\odot}$ model as it has the maximum $M_{\mathrm{TDU}}$ for the low-mass models. For the intermediate-mass models, the yield on the whole decreases with the $7 M_{\odot}$ model having the lowest yield of $2.56 \times 10^{-4} M_{\odot}$.

In Table 12, we present the final ${ }^{12} \mathrm{C} /{ }^{13} \mathrm{C}$ ratio at the surface. The ${ }^{12} \mathrm{C} /{ }^{13} \mathrm{C}$ ratio can be determined observationally and is a key observational constraint for stellar models. For the low-mass 
Table 9

An Example of the Online Data Table Providing the Elemental Yields

\begin{tabular}{|c|c|c|c|c|c|c|c|c|}
\hline Mass $^{a}$ & El. ${ }^{b}$ & $Z^{\mathrm{c}}$ & $M_{i}{ }^{\mathrm{d}}$ & $M_{\text {lost }}(i)^{\mathrm{e}}$ & $M_{0}(i)^{\mathrm{f}}$ & $\langle X(i)\rangle^{\mathrm{g}}$ & $X_{0}(i)^{\mathrm{h}}$ & $f^{\mathrm{i}}$ \\
\hline 1.0 & $n$ & 0 & $0.00 \mathrm{e}+0$ & $0.00 \mathrm{e}+0$ & $0.00 \mathrm{e}+0$ & $0.00 \mathrm{e}+0$ & $0.00 \mathrm{e}+0$ & $0.00 \mathrm{e}+0$ \\
\hline 1.0 & $\mathrm{H}$ & 1 & $-8.78 \mathrm{e}-3$ & $2.41 \mathrm{e}-1$ & $2.49 \mathrm{e}-1$ & $7.23 e-1$ & $7.49 \mathrm{e}-1$ & $-1.56 \mathrm{e}-2$ \\
\hline 1.0 & $\mathrm{He}$ & 2 & $8.56 e-3$ & $9.18 \mathrm{e}-2$ & $8.32 \mathrm{e}-2$ & $2.76 \mathrm{e}-1$ & $2.50 \mathrm{e}-1$ & $4.25 \mathrm{e}-2$ \\
\hline 1.0 & $\mathrm{Li}$ & 3 & $9.56 \mathrm{e}-11$ & $2.87 \mathrm{e}-10$ & $1.91 \mathrm{e}-10$ & $8.63 e-10$ & $5.75 \mathrm{e}-10$ & $1.76 \mathrm{e}-1$ \\
\hline 1.0 & $\mathrm{C}$ & 6 & $1.92 \mathrm{e}-4$ & $2.49 \mathrm{e}-4$ & $5.77 e-5$ & $7.49 \mathrm{e}-4$ & $1.73 e-4$ & $6.36 \mathrm{e}-1$ \\
\hline 1.0 & $\mathrm{~N}$ & 7 & $1.95 \mathrm{e}-5$ & $3.64 e-5$ & $1.69 e-5$ & $1.09 \mathrm{e}-4$ & $5.07 \mathrm{e}-5$ & $3.34 \mathrm{e}-1$ \\
\hline 1.0 & $\mathrm{O}$ & 8 & $3.59 \mathrm{e}-6$ & $1.43 \mathrm{e}-4$ & $1.40 \mathrm{e}-4$ & $4.31 \mathrm{e}-4$ & $4.20 \mathrm{e}-4$ & $1.10 \mathrm{e}-2$ \\
\hline 1.0 & $\mathrm{~F}$ & 9 & $5.63 e-9$ & $1.38 \mathrm{e}-8$ & $8.13 e-9$ & $4.13 e-8$ & $2.44 \mathrm{e}-8$ & $2.29 \mathrm{e}-1$ \\
\hline 1.0 & $\mathrm{Ne}$ & 10 & $5.05 e-6$ & $3.56 e-5$ & $3.06 e-5$ & $1.07 \mathrm{e}-4$ & $9.18 \mathrm{e}-5$ & $6.64 \mathrm{e}-2$ \\
\hline 1.0 & $\mathrm{Na}$ & 11 & $1.37 \mathrm{e}-7$ & $7.87 e-7$ & $6.50 \mathrm{e}-7$ & $2.36 e-6$ & $1.95 \mathrm{e}-6$ & $8.29 \mathrm{e}-2$ \\
\hline 1.0 & $\mathrm{Mg}$ & 12 & $-2.17 e-8$ & $1.72 \mathrm{e}-5$ & $1.73 e-5$ & $5.18 \mathrm{e}-5$ & $5.19 \mathrm{e}-5$ & $-5.45 e-4$ \\
\hline
\end{tabular}

Notes. Yields of elements up to and including Po are available online for all models.

a Initial stellar mass.

b Species $i$.

c Mass number.

${ }^{\mathrm{d}}$ Net stellar yield as defined in Equation (4).

e Amount of the species $i$ in the wind lost from the star.

$\mathrm{f}$ Total mass expelled during the stellar lifetime multiplied by the initial mass fraction.

g Average mass fraction of species $i$ in the wind.

${ }^{\mathrm{h}}$ Initial mass fraction of species $i$.

${ }^{\mathrm{i}}$ Production factor $f$ defined as $\log _{10}\left[\langle X(i)\rangle / X_{0}(i)\right]$.

(This table is available in its entirety in a machine-readable form in the online journal. A portion is shown here for guidance regarding its form and content.)

Table 10

An Example of the Online Data Table Providing the Final Surface Abundances (in $Y$ ) for Each Isotope

\begin{tabular}{|c|c|c|c|c|c|c|c|}
\hline Species & $A$ & $1 M_{\odot}$ & $1.25 M_{\odot}$ & $1.5 M_{\odot}$ & $2 M_{\odot}$ & $2.25 M_{\odot}$ & $2.5 M_{\odot}$ \\
\hline$p$ & 1 & $7.18 \mathrm{e}-1$ & $7.09 \mathrm{e}-1$ & $7.03 e-1$ & $6.81 \mathrm{e}-1$ & $6.76 \mathrm{e}-1$ & $6.85 \mathrm{e}-1$ \\
\hline$d$ & 2 & $5.92 \mathrm{e}-7$ & $5.59 \mathrm{e}-7$ & $4.67 \mathrm{e}-7$ & $1.03 \mathrm{e}-8$ & $9.32 \mathrm{e}-10$ & $1.07 \mathrm{e}-13$ \\
\hline${ }^{3} \mathrm{He}$ & 3 & $9.00 \mathrm{e}-5$ & $8.87 \mathrm{e}-5$ & $8.71 \mathrm{e}-5$ & $6.26 \mathrm{e}-5$ & $5.33 e-5$ & $5.01 \mathrm{e}-5$ \\
\hline${ }^{4} \mathrm{He}$ & 4 & $7.00 \mathrm{e}-2$ & $7.15 \mathrm{e}-2$ & $7.21 \mathrm{e}-2$ & $7.55 e-2$ & $7.63 e-2$ & $7.44 \mathrm{e}-2$ \\
\hline${ }^{7} \mathrm{Li}$ & 7 & $2.89 \mathrm{e}-10$ & $1.03 \mathrm{e}-10$ & $6.62 \mathrm{e}-11$ & $3.41 \mathrm{e}-11$ & $2.00 \mathrm{e}-11$ & $3.45 \mathrm{e}-12$ \\
\hline${ }^{7} \mathrm{Be}$ & 7 & $0.00 \mathrm{e}+0$ & $0.00 \mathrm{e}+0$ & $5.81 \mathrm{e}-31$ & $0.00 \mathrm{e}+0$ & $2.07 e-38$ & $7.41 \mathrm{e}-32$ \\
\hline${ }^{8} \mathrm{~B}$ & 8 & $4.60 \mathrm{e}-26$ & $2.89 \mathrm{e}-26$ & $1.92 \mathrm{e}-27$ & $7.72 \mathrm{e}-29$ & $2.97 \mathrm{e}-29$ & $3.52 \mathrm{e}-30$ \\
\hline${ }^{12} \mathrm{C}$ & 12 & $8.22 \mathrm{e}-5$ & $2.62 \mathrm{e}-4$ & $5.39 \mathrm{e}-4$ & $1.09 \mathrm{e}-3$ & $1.23 \mathrm{e}-3$ & $1.15 \mathrm{e}-3$ \\
\hline${ }^{13} \mathrm{C}$ & 13 & $5.15 \mathrm{e}-7$ & $4.55 \mathrm{e}-7$ & $4.37 \mathrm{e}-7$ & $4.07 \mathrm{e}-7$ & $3.94 \mathrm{e}-7$ & $4.19 \mathrm{e}-7$ \\
\hline${ }^{14} \mathrm{C}$ & 14 & $1.32 \mathrm{e}-20$ & $2.03 e-19$ & $2.27 e-13$ & $2.21 \mathrm{e}-12$ & $3.62 \mathrm{e}-12$ & $1.72 \mathrm{e}-11$ \\
\hline${ }^{13} \mathrm{~N}$ & 13 & $0.00 \mathrm{e}+0$ & $0.00 \mathrm{e}+0$ & $0.00 \mathrm{e}+0$ & $0.00 \mathrm{e}+0$ & $0.00 \mathrm{e}+0$ & $0.00 \mathrm{e}+0$ \\
\hline${ }^{14} \mathrm{~N}$ & 14 & $1.01 \mathrm{e}-5$ & $1.03 e-5$ & $1.06 \mathrm{e}-5$ & $1.17 \mathrm{e}-5$ & $1.15 \mathrm{e}-5$ & $1.04 \mathrm{e}-5$ \\
\hline${ }^{15} \mathrm{~N}$ & 15 & $5.00 \mathrm{e}-9$ & $6.38 \mathrm{e}-9$ & $7.45 \mathrm{e}-9$ & $5.89 \mathrm{e}-9$ & $4.25 e-9$ & $3.26 \mathrm{e}-9$ \\
\hline
\end{tabular}

Notes. Abundances of isotopes up to and including ${ }^{70} \mathrm{Zn}$ are available online for all models. $Y=X / A$ where $X$ is the mass fraction and $A$ is the atomic mass.

(This table is available in its entirety in a machine-readable form in the online journal. A portion is shown here for guidance regarding its form and content.)

models, the ${ }^{12} \mathrm{C} /{ }^{13} \mathrm{C}$ ratio increases as TDU brings ${ }^{12} \mathrm{C}$ synthesized from He-burning to the surface. The $3 M_{\odot}$ model has very inefficient $\mathrm{HBB}$ which results in a final ${ }^{12} \mathrm{C} /{ }^{13} \mathrm{C}$ ratio of 89. As a result of the $\mathrm{CN}$ cycle during $\mathrm{HBB}$ the ${ }^{12} \mathrm{C} /{ }^{13} \mathrm{C}$ ratio for the intermediate-mass models reaches an equilibrium value of approximately three. This is in agreement with the value found by Frost et al. (1998). The final ${ }^{12} \mathrm{C} /{ }^{13} \mathrm{C}$ ratio is greater than three as it increases as a result of $\mathrm{HBB}$ ceasing while TDU continues.

All the models become carbon-rich with the $\mathrm{C} / \mathrm{O}$ ratio increasing to above unity. The low-mass models have a high final $\mathrm{C} / \mathrm{O}$ ratio. This is because the surface abundance of $\mathrm{O}$ only increases slightly compared to the increase of C. Despite the more massive models having a higher $T_{\text {bce }}$ and more efficient
$\mathrm{HBB}$, the $4 M_{\odot}$ model has the lowest $\mathrm{C} / \mathrm{O}$ ratio of 3.38 of all the intermediate-mass models.

The final surface abundance of $\mathrm{N}$ is reasonably constant for the low-mass models, with the increase in $[\mathrm{N} / \mathrm{Fe}]$ to $\sim 0.5$ a result of FDU. The $\mathrm{N}$ yield increases with increasing initial mass from $1.95 \times 10^{-5} M_{\odot}$ for the $1 M_{\odot}$ model to $2.34 \times 10^{-4} M_{\odot}$ for the $3 M_{\odot}$ model. The intermediate-mass models experience HBB and, as such, have a higher final surface abundance of $\mathrm{N}$ compared to the low-mass models. The $4 M_{\odot}$ model has the highest $[\mathrm{N} / \mathrm{Fe}]$ ratio (with a value of 2.62) and $\mathrm{N}$ yield $\left(5.94 \times 10^{-2} M_{\odot}\right)$ of all the models. This is a result of the $4 M_{\odot}$ model having the largest amount of material brought to the surface, which provides additional primary $\mathrm{C}$ to be converted to $\mathrm{N}$. 

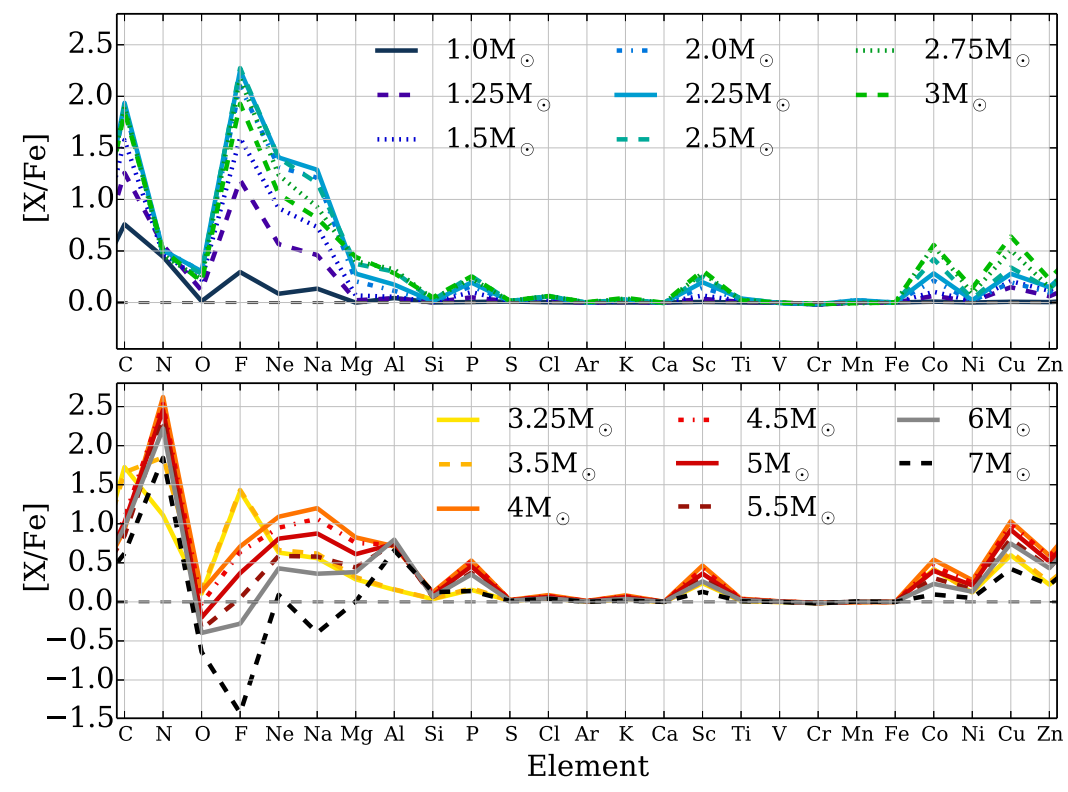

Figure 10. Final surface abundances relative to $\mathrm{Fe}$ for each of the models for the light elements from $\mathrm{C}$ to $\mathrm{Zn}$. The elements are ordered by increasing atomic number. (A color version of this figure is available in the online journal.)

Table 11

An Example of the Online Data Table Providing the Final Surface Abundance of Each Element

\begin{tabular}{lccccccc}
\hline \hline Mass $^{\mathrm{a}}$ & $\mathrm{El}^{\mathrm{b}}$ & $Z^{\mathrm{c}}$ & $\log \epsilon(X)^{\mathrm{d}}$ & \multicolumn{1}{c}{$[\mathrm{X} / \mathrm{H}]^{\mathrm{e}}$} & {$[\mathrm{X} / \mathrm{Fe}]^{\mathrm{e}}$} & \multicolumn{1}{c}{$[\mathrm{X} / \mathrm{O}]^{\mathrm{e}}$} & \multicolumn{1}{c}{$X(i)^{\mathrm{f}}$} \\
\hline 1.0 & $\mathrm{H}$ & 1 & $1.20 \mathrm{e}+1$ & $0.00 \mathrm{e}+0$ & $0.00 \mathrm{e}+0$ & $0.00 \mathrm{e}+0$ & $7.24 \mathrm{e}-1$ \\
1.0 & $\mathrm{He}$ & 2 & $1.10 \mathrm{e}+1$ & $5.97 \mathrm{e}-2$ & $1.23 \mathrm{e}+0$ & $1.21 \mathrm{e}+0$ & $2.81 \mathrm{e}-1$ \\
1.0 & $\mathrm{Li}$ & 3 & $2.60 \mathrm{e}+0$ & $-6.56 \mathrm{e}-1$ & $5.11 \mathrm{e}-1$ & $4.99 \mathrm{e}-1$ & $2.00 \mathrm{e}-9$ \\
1.0 & $\mathrm{Be}$ & 4 & $0.00 \mathrm{e}+0$ & $-1.30 \mathrm{e}+0$ & $0.00 \mathrm{e}+0$ & $0.00 \mathrm{e}+0$ & $0.00 \mathrm{e}+0$ \\
1.0 & $\mathrm{~B}$ & 5 & $0.00 \mathrm{e}+0$ & $-2.79 \mathrm{e}+0$ & $0.00 \mathrm{e}+0$ & $0.00 \mathrm{e}+0$ & $4.97 \mathrm{e}-25$ \\
1.0 & $\mathrm{C}$ & 6 & $8.06 \mathrm{e}+0$ & $-4.08 \mathrm{e}-1$ & $7.59 \mathrm{e}-1$ & $7.46 \mathrm{e}-1$ & $9.93 \mathrm{e}-4$ \\
1.0 & $\mathrm{~N}$ & 7 & $7.15 \mathrm{e}+0$ & $-7.23 \mathrm{e}-1$ & $4.44 \mathrm{e}-1$ & $4.31 \mathrm{e}-1$ & $1.41 \mathrm{e}-4$ \\
1.0 & $\mathrm{O}$ & 8 & $7.58 \mathrm{e}+0$ & $-1.15 \mathrm{e}+0$ & $1.27 \mathrm{e}-2$ & $0.00 \mathrm{e}+0$ & $4.32 \mathrm{e}-4$ \\
1.0 & $\mathrm{~F}$ & 9 & $3.55 \mathrm{e}+0$ & $-8.71 \mathrm{e}-1$ & $2.96 \mathrm{e}-1$ & $2.83 \mathrm{e}-1$ & $4.82 \mathrm{e}-8$ \\
1.0 & $\mathrm{Ne}$ & 10 & $6.89 \mathrm{e}+0$ & $-1.08 \mathrm{e}+0$ & $8.51 \mathrm{e}-2$ & $7.24 \mathrm{e}-2$ & $1.12 \mathrm{e}-4$ \\
1.0 & $\mathrm{Na}$ & 11 & $5.21 \mathrm{e}+0$ & $-1.03 \mathrm{e}+0$ & $1.35 \mathrm{e}-1$ & $1.22 \mathrm{e}-1$ & $2.66 \mathrm{e}-6$ \\
1.0 & $\mathrm{Mg}$ & 12 & $6.47 \mathrm{e}+0$ & $-1.17 \mathrm{e}+0$ & $-1.16 \mathrm{e}-3$ & $-1.39 \mathrm{e}-2$ & $5.17 \mathrm{e}-5$ \\
\hline
\end{tabular}

Notes. Final surface abundances of elements up to and including Po are available online for all models.

${ }^{\mathrm{a}}$ Initial stellar mass.

${ }^{\mathrm{b}}$ Element.

c Atomic number.

${ }^{\mathrm{d}} \log \epsilon(X)=\log _{10}\left(N_{A} / N_{H}\right)+12$ where $N_{A}$ and $N_{H}$ are abundances of element $\mathrm{A}$ and $\mathrm{H}$.

${ }^{\mathrm{e}}[\mathrm{X} / \mathrm{Y}]=\log _{10}\left(N_{X} / N_{Y}\right)_{\star}-\log _{10}\left(N_{X} / N_{Y}\right)_{\odot}$ where $N_{X}$ and $N_{Y}$ are the abundances of elements $\mathrm{X}$ and $\mathrm{Y}$.

${ }^{\mathrm{f}}$ Mass fraction of element.

(This table is available in its entirety in a machine-readable form in the online journal. A portion is shown here for guidance regarding its form and content.)

The low-mass models between 2 and $2.5 M_{\odot}$ have a similar final surface abundance of $\mathrm{O}$, with an $[\mathrm{O} / \mathrm{Fe}]$ ratio of around 0.3 . The final $\mathrm{O}$ abundance is lower for the intermediate-mass models due to its destruction from HBB. For the models with a mass of $4.5 M_{\odot}$ and higher, the $[\mathrm{O} / \mathrm{Fe}]$ abundances are negative, down to -0.64 for the $7 M_{\odot}$ model. As expected, the $7 M_{\odot}$ model has the lowest net yield of $\mathrm{O}$ with $-2.20 \times 10^{-3} M_{\odot}$. The highest yield of $6.34 \times 10^{-4} M_{\odot}$ occurs for the $2.5 M_{\odot}$ model.

There is only one stable isotope of $\mathrm{F}\left({ }^{19} \mathrm{~F}\right)$ which is produced through the ${ }^{15} \mathrm{~N}(\alpha, \gamma){ }^{19} \mathrm{~F}$ reaction in the He-intershell (Jorissen et al. 1992; Mowlavi et al. 1996; Lugaro et al. 2004; Abia et al. 2009). The F synthesized during the preceding TP is mixed to the surface during TDU. The final $F$ surface abundance increases for each model, with $[\mathrm{F} / \mathrm{Fe}]$ up to 2.27 for the $2.5 M_{\odot}$, before decreasing to sub-solar values for the 6 and $7 M_{\odot}$ models. The decrease in $\mathrm{F}$ is caused by the destruction of ${ }^{19} \mathrm{~F}$ through $\alpha$ capture to produce ${ }^{22} \mathrm{Ne}$. In the more massive models, the $\mathrm{F}$ yield also decreases as temperatures during $\mathrm{HBB}$ allow for the destruction of $\mathrm{F}$ to take place via the ${ }^{19} \mathrm{~F}(p, \alpha){ }^{16} \mathrm{O}$ reaction. The 5, $5.5,6$, and $7 M_{\odot}$ models have a negative F yield, with the lowest net yield of $-1.41 \times 10^{-7} M_{\odot}$ occurring for the $7 M_{\odot}$ model.

\subsection{2. $\mathrm{Ne}, \mathrm{Na}, \mathrm{Mg}$, and $\mathrm{Al}$}

Only $\mathrm{Ne}$ and $\mathrm{Na}$ are noticeably affected by FDU and SDU whereas the surface abundances of $\mathrm{Mg}$ and $\mathrm{Al}$ do not change during FDU for the low-mass models and changes by only $35 \%$ for ${ }^{27} \mathrm{Al}$ for the intermediate-mass models. Of all the models, the $2.25 M_{\odot}$ has the highest final surface abundance of $[\mathrm{Ne} / \mathrm{Fe}]$ and $[\mathrm{Na} / \mathrm{Fe}]$ as a result of FDU and TDU with 1.41 and 1.29, respectively (Table 12). The $\mathrm{Ne}-\mathrm{Na}$ chain is activated during HBB for the intermediate-mass models and the main result of the $\mathrm{Ne}-\mathrm{Na}$ chain is the production of ${ }^{23} \mathrm{Na}$, the only stable isotope of $\mathrm{Na}$, at the expense of ${ }^{22} \mathrm{Ne}$. The $4 M_{\odot}$ model has the highest yield of $\mathrm{Na}$ with $7.57 \times 10^{-5} M_{\odot}$. The $7 M_{\odot}$ model is the only model with a negative $\mathrm{Na}$ yield.

The highest final surface abundances of $\mathrm{Mg}$ and $\mathrm{Al}$ occur for the 4 and $6 M_{\odot}$ models, respectively. This is also reflected in the net yields. The yield of $\mathrm{Mg}$ increases with increasing initial mass before decreasing once the peak yield of $7.53 \times 10^{-4} M_{\odot}$ is reached for the $4 M_{\odot}$ model. The 3.25 and $3.5 M_{\odot}$ models are exceptions to this trend. This is because the models (compared to lower and higher mass models) have less TDU, the absence of a PMZ resulting in fewer neutron captures onto ${ }^{24} \mathrm{Mg}$, lower activation of the ${ }^{22} \mathrm{Ne}+\alpha$ reaction compared to intermediate-mass models, and the $\mathrm{Mg}-\mathrm{Al}$ chain is not activated. The production of $\mathrm{Al}$ increases with increasing mass for the intermediate-mass models; however, the $7 M_{\odot}$ model has a lower Al yield than the $6 M_{\odot}$ due to HBB temperatures being high enough for the production of ${ }^{28} \mathrm{Si}$ to occur at the expense of ${ }^{27} \mathrm{Al}$. 
Table 12

Final Surface ${ }^{4} \mathrm{He}$ Mass Fraction, C/O Ratio, ${ }^{12} \mathrm{C} /{ }^{13} \mathrm{C}$ Ratio, and $[\mathrm{X} / \mathrm{Fe}]$ Ratios for Selected Light Elements

\begin{tabular}{|c|c|c|c|c|c|c|c|c|c|c|c|}
\hline Mass & ${ }^{4} \mathrm{He}$ & $\mathrm{C} / \mathrm{O}$ & ${ }^{12} \mathrm{C} /{ }^{13} \mathrm{C}$ & {$[\mathrm{C} / \mathrm{Fe}]$} & {$[\mathrm{N} / \mathrm{Fe}]$} & {$[\mathrm{O} / \mathrm{Fe}]$} & {$[\mathrm{F} / \mathrm{Fe}]$} & {$[\mathrm{Ne} / \mathrm{Fe}]$} & {$[\mathrm{Na} / \mathrm{Fe}]$} & {$[\mathrm{Mg} / \mathrm{Fe}]$} & {$[\mathrm{Al} / \mathrm{Fe}]$} \\
\hline 1.00 & 0.28 & 3.07 & 160 & 0.76 & 0.44 & 0.01 & 0.30 & 0.09 & 0.13 & 0.00 & 0.04 \\
\hline 1.25 & 0.29 & 7.67 & 575 & 1.26 & 0.45 & 0.12 & 1.19 & 0.57 & 0.40 & 0.02 & 0.04 \\
\hline 1.50 & 0.29 & 12.47 & 1232 & 1.58 & 0.47 & 0.22 & 1.60 & 0.91 & 0.73 & 0.07 & 0.05 \\
\hline 2.00 & 0.30 & 21.04 & 2687 & 1.88 & 0.51 & 0.30 & 2.10 & 1.31 & 1.21 & 0.21 & 0.11 \\
\hline 2.25 & 0.31 & 23.80 & 3129 & 1.94 & 0.51 & 0.30 & 2.25 & 1.41 & 1.29 & 0.28 & 0.17 \\
\hline 2.50 & 0.30 & 23.47 & 2752 & 1.91 & 0.46 & 0.28 & 2.27 & 1.40 & 1.16 & 0.37 & 0.30 \\
\hline 2.75 & 0.29 & 26.02 & 2355 & 1.90 & 0.46 & 0.22 & 2.17 & 1.24 & 0.93 & 0.40 & 0.32 \\
\hline 3.00 & 0.29 & 24.82 & 89 & 1.85 & 0.51 & 0.19 & 1.92 & 1.05 & 0.81 & 0.44 & 0.28 \\
\hline 3.25 & 0.28 & 22.93 & 11 & 1.72 & 1.11 & 0.10 & 1.41 & 0.63 & 0.56 & 0.29 & 0.15 \\
\hline 3.50 & 0.29 & 18.32 & 6.27 & 1.66 & 1.85 & 0.13 & 1.43 & 0.66 & 0.62 & 0.31 & 0.16 \\
\hline 4.00 & 0.34 & 3.38 & 5.97 & 0.91 & 2.62 & 0.12 & 0.70 & 1.08 & 1.19 & 0.82 & 0.70 \\
\hline 4.50 & 0.35 & 6.91 & 7.99 & 1.07 & 2.53 & -0.03 & 0.63 & 0.94 & 1.06 & 0.75 & 0.72 \\
\hline 5.00 & 0.36 & 9.07 & 7.56 & 1.01 & 2.45 & -0.20 & 0.36 & 0.80 & 0.87 & 0.61 & 0.74 \\
\hline 5.50 & 0.36 & 8.69 & 6.11 & 0.84 & 2.34 & -0.35 & 0.04 & 0.58 & 0.58 & 0.44 & 0.75 \\
\hline 6.00 & 0.36 & 12.58 & 8.53 & 0.96 & 2.22 & -0.40 & -0.28 & 0.43 & 0.36 & 0.38 & 0.79 \\
\hline 7.00 & 0.36 & 10.09 & 6.65 & 0.62 & 1.84 & -0.64 & -1.42 & 0.09 & -0.40 & 0.00 & 0.66 \\
\hline
\end{tabular}

Table 13

Final Surface Abundances for Select Neutron-capture Elements and s-process Indicators

\begin{tabular}{|c|c|c|c|c|c|c|c|c|c|c|}
\hline Mass & {$[\mathrm{Rb} / \mathrm{Fe}]$} & {$[\mathrm{Sr} / \mathrm{Fe}]$} & {$[\mathrm{Y} / \mathrm{Fe}]$} & {$[\mathrm{Zr} / \mathrm{Fe}]$} & {$[\mathrm{Ba} / \mathrm{Fe}]$} & {$[\mathrm{La} / \mathrm{Fe}]$} & {$[\mathrm{Ce} / \mathrm{Fe}]$} & {$[\mathrm{Pb} / \mathrm{Fe}]$} & {$[l s / \mathrm{Fe}]$} & {$[h s / \mathrm{Fe}]$} \\
\hline 1.00 & 0.04 & 0.24 & 0.23 & 0.22 & 0.20 & 0.21 & 0.29 & 1.28 & 0.23 & 0.18 \\
\hline 1.25 & 0.25 & 0.68 & 0.74 & 0.83 & 1.27 & 1.27 & 1.34 & 2.31 & 0.75 & 1.18 \\
\hline 1.50 & 0.52 & 1.07 & 1.10 & 1.18 & 1.60 & 1.62 & 1.72 & 2.68 & 1.11 & 1.55 \\
\hline 2.00 & 0.70 & 1.34 & 1.42 & 1.53 & 2.02 & 2.03 & 2.14 & 2.95 & 1.43 & 1.95 \\
\hline 2.25 & 0.89 & 1.43 & 1.52 & 1.64 & 2.13 & 2.14 & 2.26 & 2.99 & 1.53 & 2.06 \\
\hline 2.50 & 1.20 & 1.42 & 1.54 & 1.68 & 2.12 & 2.13 & 2.26 & 2.94 & 1.55 & 2.03 \\
\hline 2.75 & 1.05 & 1.17 & 1.30 & 1.47 & 1.85 & 1.85 & 2.00 & 2.75 & 1.31 & 1.75 \\
\hline 3.00 & 1.00 & 0.98 & 1.08 & 1.27 & 1.64 & 1.59 & 1.75 & 2.56 & 1.11 & 1.51 \\
\hline 3.25 & 0.84 & 0.60 & 0.53 & 0.47 & 0.16 & 0.08 & 0.07 & 0.04 & 0.53 & 0.03 \\
\hline 3.50 & 0.89 & 0.65 & 0.58 & 0.51 & 0.18 & 0.10 & 0.09 & 0.04 & 0.58 & 0.04 \\
\hline 4.00 & 1.65 & 1.45 & 1.42 & 1.40 & 0.96 & 0.72 & 0.68 & 0.29 & 1.41 & 0.58 \\
\hline 4.50 & 1.65 & 1.45 & 1.43 & 1.41 & 0.97 & 0.73 & 0.69 & 0.31 & 1.42 & 0.59 \\
\hline 5.00 & 1.55 & 1.37 & 1.34 & 1.32 & 0.88 & 0.64 & 0.59 & 0.25 & 1.33 & 0.51 \\
\hline 5.50 & 1.43 & 1.25 & 1.23 & 1.21 & 0.79 & 0.57 & 0.52 & 0.20 & 1.23 & 0.44 \\
\hline 6.00 & 1.45 & 1.25 & 1.22 & 1.19 & 0.75 & 0.52 & 0.46 & 0.16 & 1.22 & 0.40 \\
\hline 7.00 & 1.07 & 0.83 & 0.79 & 0.75 & 0.35 & 0.20 & 0.17 & 0.04 & 0.79 & 0.13 \\
\hline
\end{tabular}

\subsubsection{Other Light Elements}

There is a slight enhancement of up to approximately 0.15 dex for $\mathrm{Si}, \mathrm{S}, \mathrm{Cl}$, and Ar. The $7 M_{\odot}$ model produces the most $\mathrm{Si}$ with a net yield of $7.73 \times 10^{-5} M_{\odot}$ (Table 7 ). $[\mathrm{P} / \mathrm{Fe}]$ increases by around $0.5 \mathrm{dex}$ for the intermediate-mass models with the maximum enhancement occurring for the $4 M_{\odot}$ model. Sc is produced with the $4 M_{\odot}$ model showing the largest enhancement of $[\mathrm{Sc} / \mathrm{Fe}](\approx 0.4 \mathrm{dex})$. Of the Fe-group elements, $\mathrm{Cu}$ is enhanced the most in the $4 M_{\odot}$ model increasing by 1.03 dex for $[\mathrm{Cu} / \mathrm{Fe}]$. The abundance of $[\mathrm{Zn} / \mathrm{H}]$ has been proposed to be a good proxy of $[\mathrm{Fe} / \mathrm{H}]$ in planetary nebulae (e.g., Dinerstein \& Geballe 2001; Smith et al. 2014). The low-mass models have $[\mathrm{Zn} / \mathrm{Fe}]$ enhancements between 0 and 0.32 dex with the $1 M_{\odot}$ model having no increase and the $3 M_{\odot}$ model having the largest increase. The smallest enhancement for the intermediate-mass models occurs for the $7 M_{\odot}$ model with a $[\mathrm{Zn} / \mathrm{Fe}]$ ratio of 0.22 whereas the $4 M_{\odot}$ model has $[\mathrm{Zn} / \mathrm{Fe}]$ increase by 0.59 dex.

\subsubsection{Comparison with Cristallo et al. (2011)}

Figure 11 also presents the net yields of Cristallo et al. (2011) for each of the initial stellar masses in common with the models presented here: $1.5,2,2.5$, and $3 M_{\odot}$. For these low masses, the net yield of $\mathrm{C}$ (and $\mathrm{O}$ ) follows the total amount of material mixed to the surface by TDU. The 2.5 and $3 M_{\odot}$ models of Cristallo et al. (2011) have a lower value of $M_{\mathrm{TDU}}$, which results in a lower $\mathrm{C}$ (and $\mathrm{O}$ ) yield in Figure 11(a); with the largest yield difference $\Delta M_{i}$ being $1.13 \times 10^{-2} M_{\odot}$ for the $3 M_{\odot}$ model.

The net yield of $\mathrm{N}$ agrees with the predictions of Cristallo et al. (2011), with the $\mathrm{N}$ yield increasing with increasing initial mass as a result of FDU. For the Cristallo et al. (2011) yields of $\mathrm{F}, \mathrm{Ne}$, and $\mathrm{Na}$, the $2 M_{\odot}$ model has the largest net yield as a result of having the largest value of $M_{\mathrm{TDU}}$. For the models presented here, the yields peak at $2.5 M_{\odot}$ except for $\mathrm{Na}$ which peaks at $2.25 M_{\odot}$. The yields of $\mathrm{Mg}$ and $\mathrm{Al}$ peak at $3 M_{\odot}$ and $2.75 M_{\odot}$, respectively.

\subsection{The Neutron-capture Elements}

In this section, we present final surface abundances and net stellar yield predictions for selected neutron-capture elements ( $\mathrm{Rb}, \mathrm{Sr}, \mathrm{Y}, \mathrm{Zr}, \mathrm{Ba}, \mathrm{La}, \mathrm{Ce}$, and $\mathrm{Pb}$ ). Table 13 presents the final surface abundances for selected neutron-capture elements and $s$-process indicators. The distribution of the final surface abundances $[\mathrm{X} / \mathrm{Fe}]$ for these elements is shown in Figure 12. 

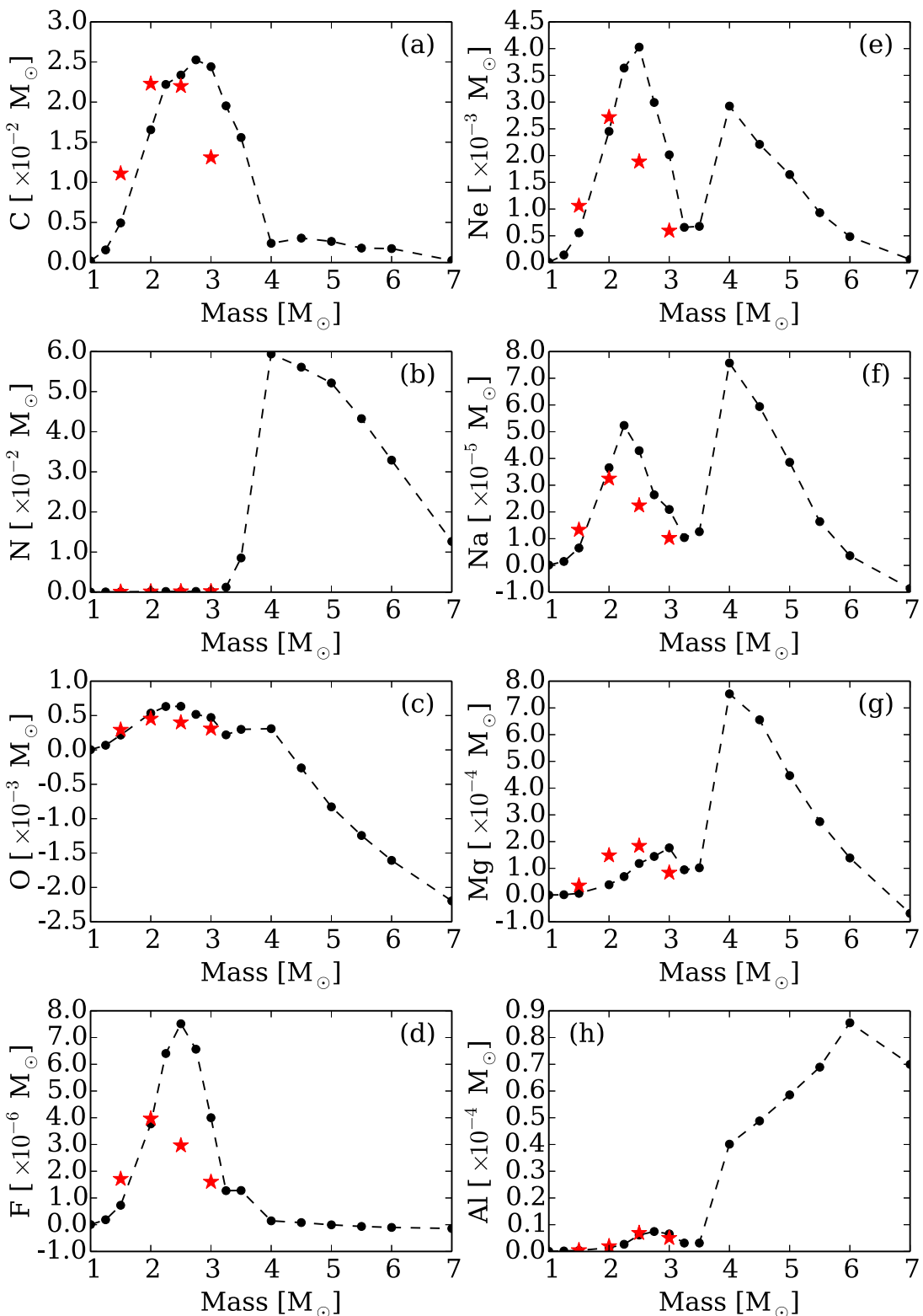

Figure 11. Net yields of select elements lighter than Si as a function of initial mass. Results from Cristallo et al. (2011) are shown as red stars.

(A color version of this figure is available in the online journal.)

As illustrated in Figure 12, the low-mass models produce a final surface abundance distribution of the neutron-capture elements that has peaks at $\mathrm{Sr}, \mathrm{Zr}, \mathrm{Ba}$, and $\mathrm{Pb}$ as discussed for the case of the $2 M_{\odot}$ model. In comparison, the intermediatemass models produce a peak at $\mathrm{Rb}$. This difference is due to a combination of the addition of a PMZ for the low-mass models and the activation of the ${ }^{22} \mathrm{Ne}$ neutron source for the intermediate-mass models.

The final surface abundance of $\mathrm{Rb}$ increases with increasing initial mass for the low-mass models, up to 1.20 for the $2 M_{\odot}$ model before decreasing slightly for the 2.75 and $3 M_{\odot}$ models. This increase is mainly the result of the mild activation of the ${ }^{22} \mathrm{Ne}$ neutron source. For the intermediate-mass models, the highest final surface abundance and yield of all the neutroncapture elements occurs for $\mathrm{Rb}$ where branching points are activated and the total neutron exposure is lower than in the low-mass models. The highest final surface abundance for $\mathrm{Rb}$ occurs for the 4 and $4.5 M_{\odot}$ models where both have a final
$[\mathrm{Rb} / \mathrm{Fe}]$ value of 1.65 . The $4.5 M_{\odot}$ model has the highest yield of Rb with $1.64 \times 10^{-7} M_{\odot}$.

Each model has a similar final abundance for $[\mathrm{Sr} / \mathrm{Fe}]$, $[\mathrm{Y} / \mathrm{Fe}]$, and $[\mathrm{Zr} / \mathrm{Fe}]$, three first $s$-process peak elements. Of the three elements, $\mathrm{Sr}$ has the lowest final abundance and $\mathrm{Zr}$ has the highest for each of the low-mass models. For the intermediate-mass models, the trend is reversed with $\mathrm{Sr}$ having the highest abundance and $\mathrm{Zr}$, the lowest of the three elements. Of the intermediate-mass models, the $4.5 M_{\odot}$ model has the highest abundances with $1.45,1.43$, and 1.41 for $\mathrm{Sr}$, $\mathrm{Y}$, and $\mathrm{Zr}$, respectively. This is also reflected in the yields of the $4.5 M_{\odot}$ model where it has the highest $\mathrm{Sr}$ and $\mathrm{Y}$ yields of all the models, $2.38 \times 10^{-7}$ and $4.85 \times 10^{-8} M_{\odot}$, respectively. For $\mathrm{Zr}$, the $2.5 M_{\odot}$ model has the highest yield of $1.19 \times 10^{-7} M_{\odot}$.

For the low mass models, the final surface abundances of $\mathrm{Ba}$, $\mathrm{La}$, and $\mathrm{Ce}$ are higher than the abundances of $\mathrm{Sr}, \mathrm{Y}$, and $\mathrm{Zr}$. The $2.25 M_{\odot}$ model has the highest final $[\mathrm{Ba} / \mathrm{Fe}],[\mathrm{La} / \mathrm{Fe}]$, and 

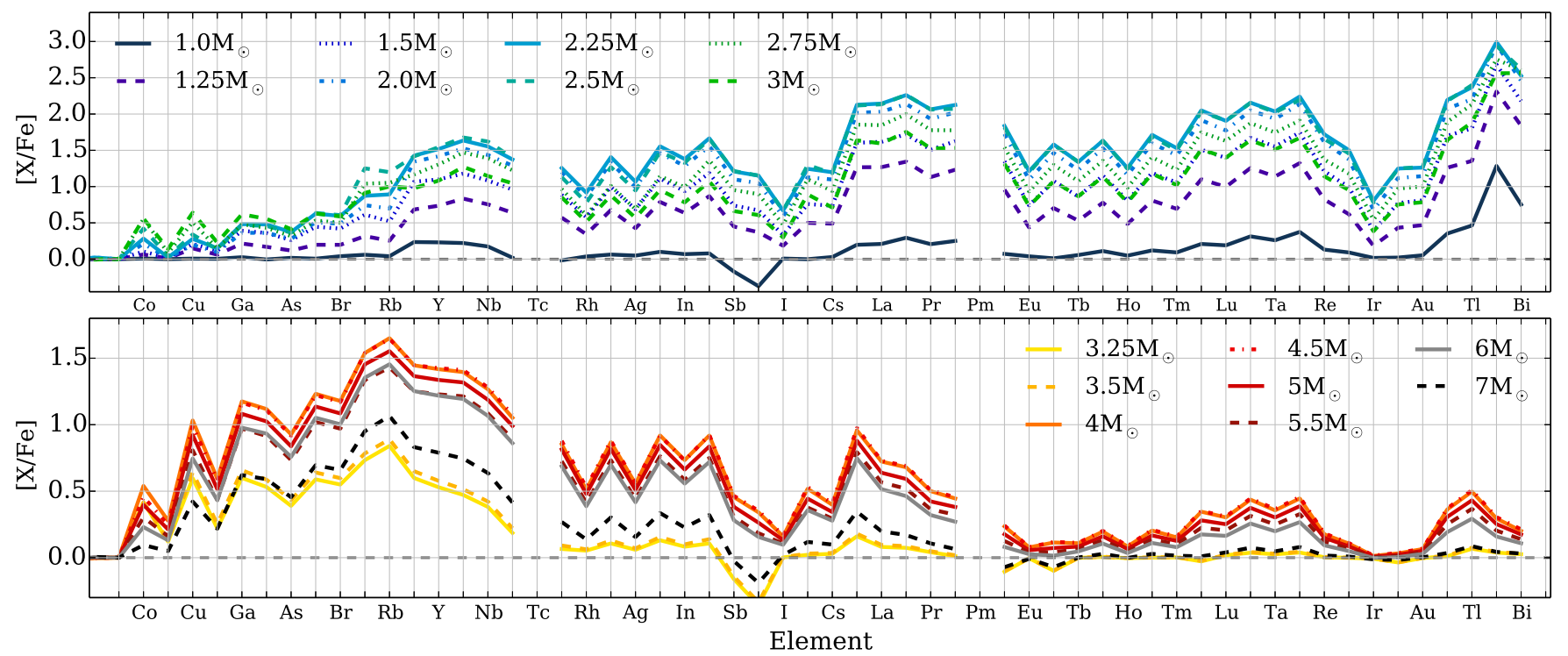

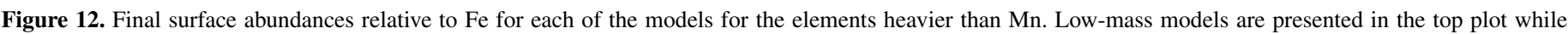

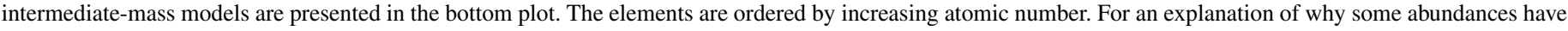
a value less than solar, see the caption of Figure 4 and Section 2.

(A color version of this figure is available in the online journal.)

$[\mathrm{Ce} / \mathrm{Fe}]$ values of all the models (see Table 8 ). The abundances of $\mathrm{Ba}, \mathrm{La}$, and $\mathrm{Ce}$ for the intermediate-mass models never reach above enhancements of $1 \mathrm{dex}$. The $4.5 M_{\odot}$ model once again has the highest final abundances of the intermediate-mass models for these three elements whereas the $7 M_{\odot}$ has the lowest. Of all the models, the $2.5 \mathrm{M}_{\odot}$ model has the highest yields for $\mathrm{Ba}, \mathrm{La}$, and $\mathrm{Ce}$ with $2.23 \times 10^{-7}, 2.28 \times 10^{-8}$, and $8.00 \times 10^{-8} M_{\odot}$, respectively.

The low-mass models produce more $\mathrm{Pb}$ compared to the intermediate-mass models (Figure 12). Once the first and second $s$-process peaks reach equilibrium, any increase in their abundance is prevented and only the abundance of $\mathrm{Pb}$ increases. The value of $[\mathrm{Pb} / \mathrm{Fe}]$ reaches a maximum value of 2.99 for the $2.25 M_{\odot}$ model. The intermediate-mass models produce minimal $\mathrm{Pb}$ as discussed in the case of the $5 M_{\odot}$ model, with the final surface abundance ranging from 0.04 for the $3.25 M_{\odot}$ model to 0.31 for the $4.5 M_{\odot}$ model. For the low-mass models, the $\mathrm{Pb}$ yield increases with increasing mass before reaching a plateau of approximately $1.7 \times 10^{-6} M_{\odot}$ for the 2.25 to $2.5 M_{\odot}$ models (see Figure 13 ). The $\mathrm{Pb}$ yield then drops below approximately a few times $10^{-9} M_{\odot}$ for the intermediate-mass models.

Figure 14 presents the distribution of the final surface abundance of the $s$-process indicators $[l s / \mathrm{Fe}],[h s / \mathrm{Fe}],[\mathrm{Pb} / \mathrm{Fe}]$, $[h s / l s],[\mathrm{Pb} / h s]$, and $[\mathrm{Rb} / \mathrm{Zr}]$ with initial mass. The $[l s / \mathrm{Fe}]$ ratio increases with increasing initial mass for the low-mass models up to $2.5 M_{\odot}$ before decreasing for the $2.75,3$, and $3.25 M_{\odot}$ models. The $[l s / \mathrm{Fe}]$ ratio then increases up to 1.43 for the $4.5 M_{\odot}$ model before decreasing again with increasing initial mass. The $[h s / \mathrm{Fe}]$ and $[\mathrm{Pb} / \mathrm{Fe}]$ values for the intermediate-mass models are less than 0.6.

Figures $14(\mathrm{~d})$ and (e) illustrate the trend of the $s$-process indicators $[h s / l s]$ and $[\mathrm{Pb} / h s]$ with initial mass. These intrinsic $s$-process indicators are independent of the amount of TDU and help constrain the neutron source and neutron exposure for the $s$-process. The low-mass models, excluding the $1 M_{\odot}$ model, plateau at approximately 0.5 for $[h s / l s]$ while the intermediate-mass plateau at a sub-solar value of around -0.8 .
For $[\mathrm{Pb} / h s]$, the low-mass models fluctuate between $\sim 0.9$ and 1.2. The intermediate-mass models have a sub-solar value of approximately -0.2 with the 3.25 and $3.5 M_{\odot}$ models having a value close to solar.

Figure 14(f) illustrates the trend of the final surface $[\mathrm{Rb} /$ $\mathrm{Zr}$ ] ratio with initial mass, where $\mathrm{Rb}$ and $\mathrm{Zr}$ are both first peak neutron-capture elements. This ratio is an indicator of the neutron density with a positive ratio resulting from higher densities produced by the ${ }^{22} \mathrm{Ne}$ neutron source. The intermediatemass models show a fairly constant $[\mathrm{Rb} / \mathrm{Zr}]$ ratio; between 0.2 and 0.4 dex. The low-mass models, however, first decrease with increasing initial mass from -0.2 for the $1 M_{\odot}$ model to -0.8 for the $2 M_{\odot}$ model, then increase to approximately $-0.3 \mathrm{dex}$ for the $3 M_{\odot}$ model. The increase in $[\mathrm{Rb} / \mathrm{Zr}]$ is due to temperatures increasing in the pulse-driven convective zone so that the ${ }^{22} \mathrm{Ne}$ neutron source is mildly activated.

\subsubsection{Comparison with Cristallo et al. (2011)}

Figure 13 also presents the yield predictions from Cristallo et al. (2011) for the $1.5,2,2.5$, and $3 M_{\odot}$ models. With the exception of $\mathrm{Rb}$ and $\mathrm{Zr}$, the Cristallo et al. (2011) yields for the $s$-process elements shown in Figure 13 have the highest value for the $2 M_{\odot}$, which has the largest $M_{\mathrm{TDU}}$ value of the four models. In contrast, the yield predictions of the models presented here peak at $2.5 M_{\odot}$ except for $\mathrm{Pb}$ which peaks at $2.25 M_{\odot}$. The yield of $\mathrm{Pb}$ reaches a plateau between $2.25 M_{\odot}$ and $2.5 M_{\odot}$. The largest difference in the yield predictions occurs for the $2.5 M_{\odot}$ model.

Figure 14 presents $s$-process indicator predictions for the Cristallo et al. (2011) models. The $s$-process indicators show a similar trend with mass between the Cristallo et al. (2011) models and the models presented here. The $[l s / \mathrm{Fe}],[h s / \mathrm{Fe}]$, and $[\mathrm{Pb} / \mathrm{Fe}]$ increase with increasing mass before reaching a peak and decreasing. The low-mass models presented here show a flat distribution for $[h s / l s]$ of approximately $0.5 \mathrm{dex}$ whereas the $[h s / l s]$ predictions of Cristallo et al. (2011) decrease with increasing initial mass, from $0.65 \mathrm{dex}$ to $0.34 \mathrm{dex}$. The values of $[\mathrm{Pb} / h s]$ fluctuate between $\sim 0.9$ and 1.2 ; however, the models 

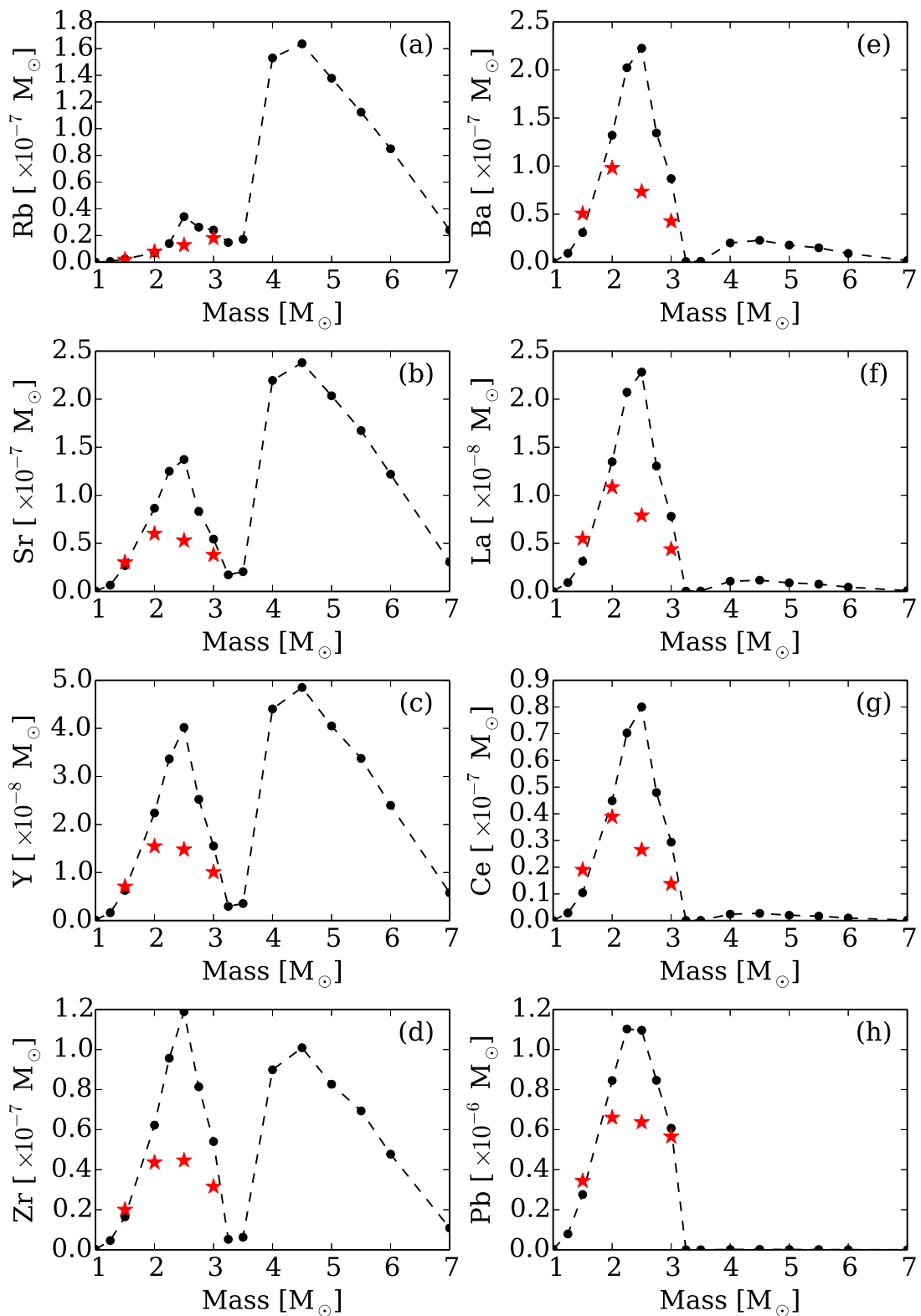

Figure 13. Net yields of select neutron-capture elements as a function of initial mass. Results from Cristallo et al. (2011) are shown as red stars.

(A color version of this figure is available in the online journal.)

of Cristallo et al. (2011) have $[\mathrm{Pb} / h s]$ mostly increasing with increasing initial mass. The $[\mathrm{Rb} / \mathrm{Zr}]$ ratios are higher for the Cristallo et al. (2011) models, due to higher peak temperatures during TPs (see Table 1). Note that the rate of the ${ }^{22} \mathrm{Ne}$ source we use from Iliadis et al. (2010) is comparable to the Jaeger et al. (2001) rate used by Cristallo et al. (2011). The final abundances of $[\mathrm{Zr} / \mathrm{Fe}]$ are lower for the Cristallo et al. (2011) models (excluding the $1.5 M_{\odot}$ model) further increasing the final $[\mathrm{Rb} / \mathrm{Zr}]$ ratio.

\section{EFFECTS OF VARYING THE MASS OF THE PMZ}

The extent in mass of the PMZ and the profile of the proton abundance in the PMZ are unknown parameters which introduce additional uncertainty into the elemental abundances and stellar yields for the low-mass AGB stellar models (Straniero et al. 2009). Here, we investigate the effect of varying the extent in mass of the PMZ, while keeping fixed the exponential profile of the proton abundance. We have computed the $3 M_{\odot}$ model using three different values for the extent in mass of the PMZ: $(0.5,1,2) \times 10^{-3} M_{\odot}$ as well as a model without the inclusion of a PMZ.

The difference in the final surface abundances of the light elements compared to the standard PMZ mass of $5 \times 10^{-4} M_{\odot}$ is shown in Figure 15. The model with a PMZ of $2 \times 10^{-3} M_{\odot}$ has the largest increase in $[\mathrm{X} / \mathrm{Fe}]$ for elements lighter than $\mathrm{Fe}$ (excluding $\mathrm{C}$ ) with respect to the standard case; the largest increase is exhibited by $\mathrm{Ne}, \mathrm{Na}$, and $\mathrm{P}$ where $\Delta[\mathrm{Ne} / \mathrm{Fe}]$, $\Delta[\mathrm{Na} / \mathrm{Fe}]$, and $\Delta[\mathrm{P} / \mathrm{Fe}]$ are approximately +0.3 . Between the model with the standard PMZ and the Cristallo et al. (2011) model, the $[\mathrm{F} / \mathrm{Fe}]$ ratio shows the largest difference of 0.4 dex. The final abundances of $[\mathrm{Ne} / \mathrm{Fe}]$ and $[\mathrm{Na} / \mathrm{Fe}]$ are also lower by approximately 0.2 dex in the Cristallo et al. model compared to our standard PMZ case.

As a larger PMZ extends over a larger mass range in the intershell, it reaches into regions of higher temperature. The 

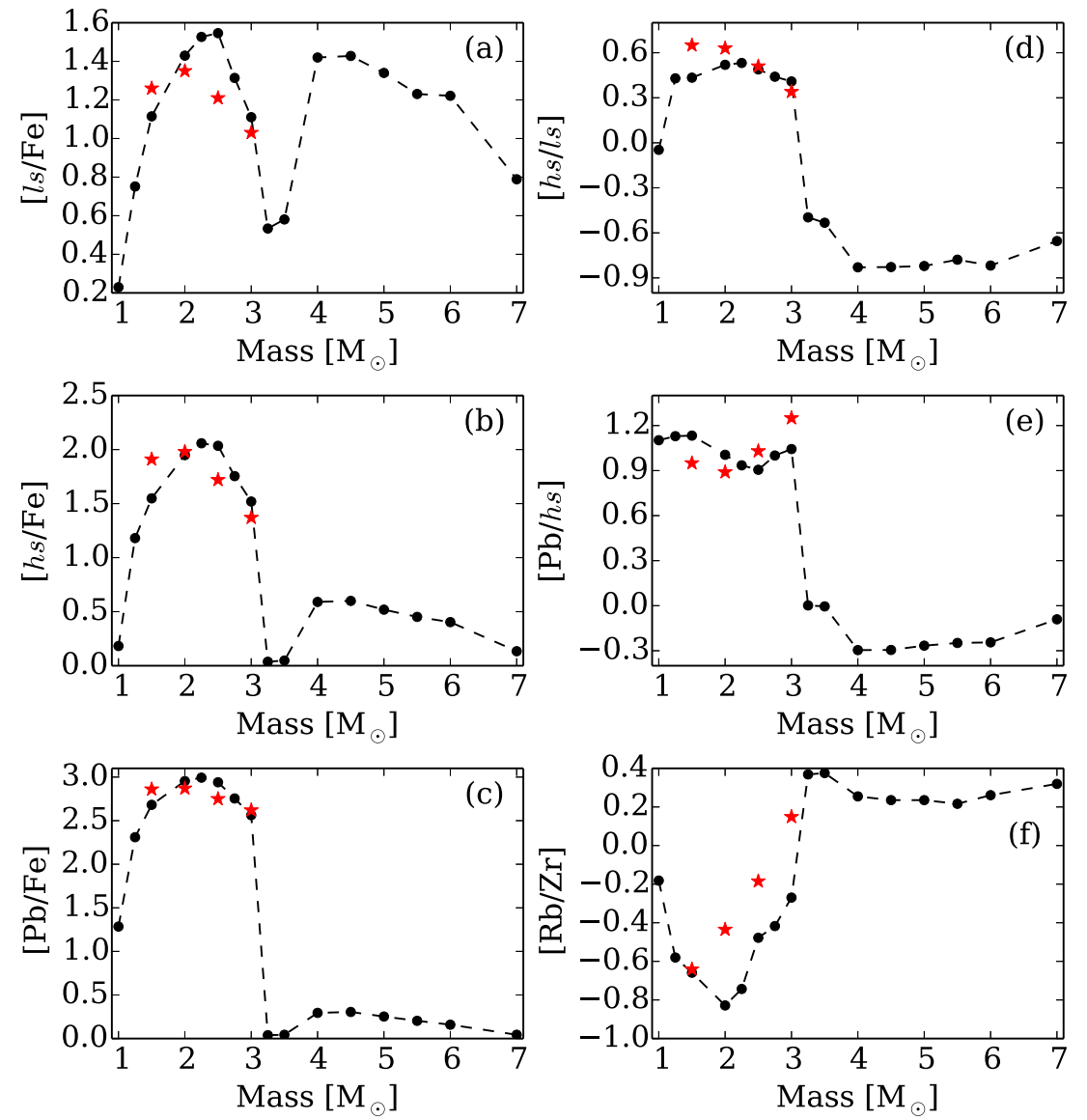

Figure 14. Distribution of $[h s / l s]$ and $[\mathrm{Pb} / h s]$ with initial mass. Results from Cristallo et al. (2011) are shown as red stars.

(A color version of this figure is available in the online journal.)
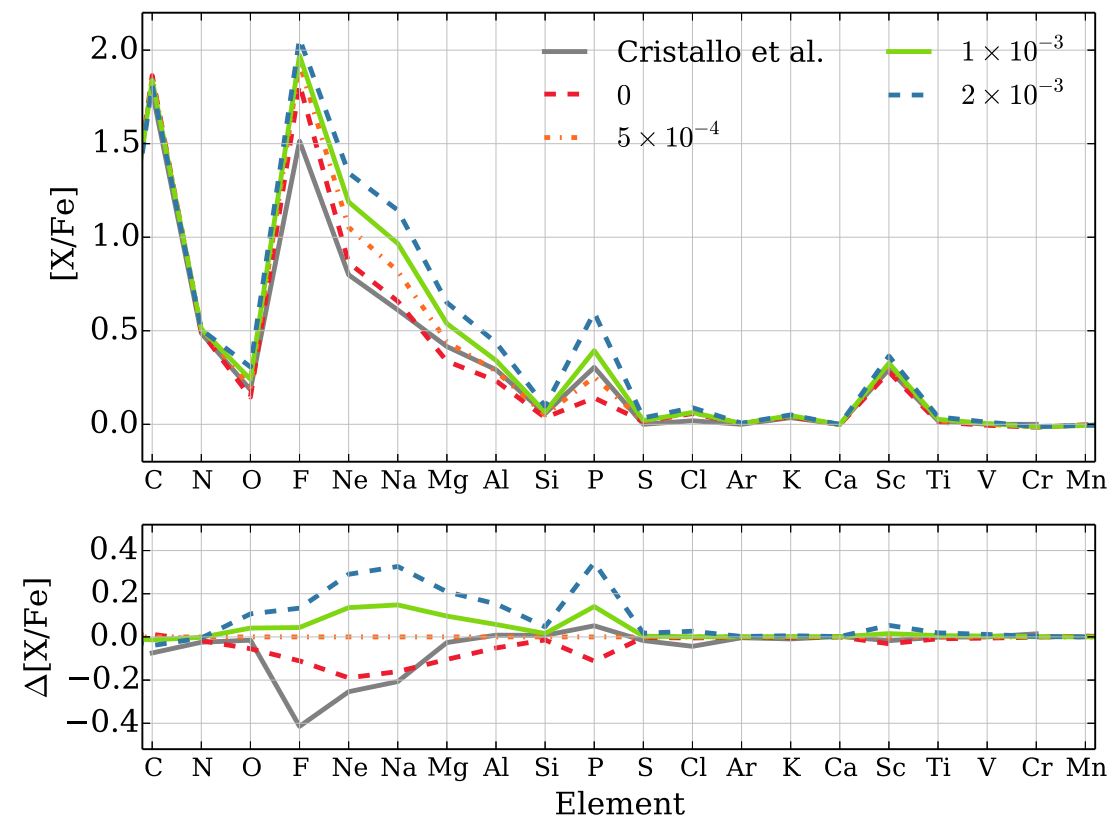

Figure 15. Final surface abundance ratios for each $3 M_{\odot}$ model with a different PMZ mass (in units of $M_{\odot}$ as indicated in the legend) for the elements lighter than Fe. The final surface abundances for the $3 M_{\odot}$ model of Cristallo et al. (2011) are also presented. The bottom panel illustrates the absolute difference between each model and the standard PMZ mass, $5 \times 10^{-4} M_{\odot}$. The elements are ordered by increasing atomic number.

(A color version of this figure is available in the online journal.) 

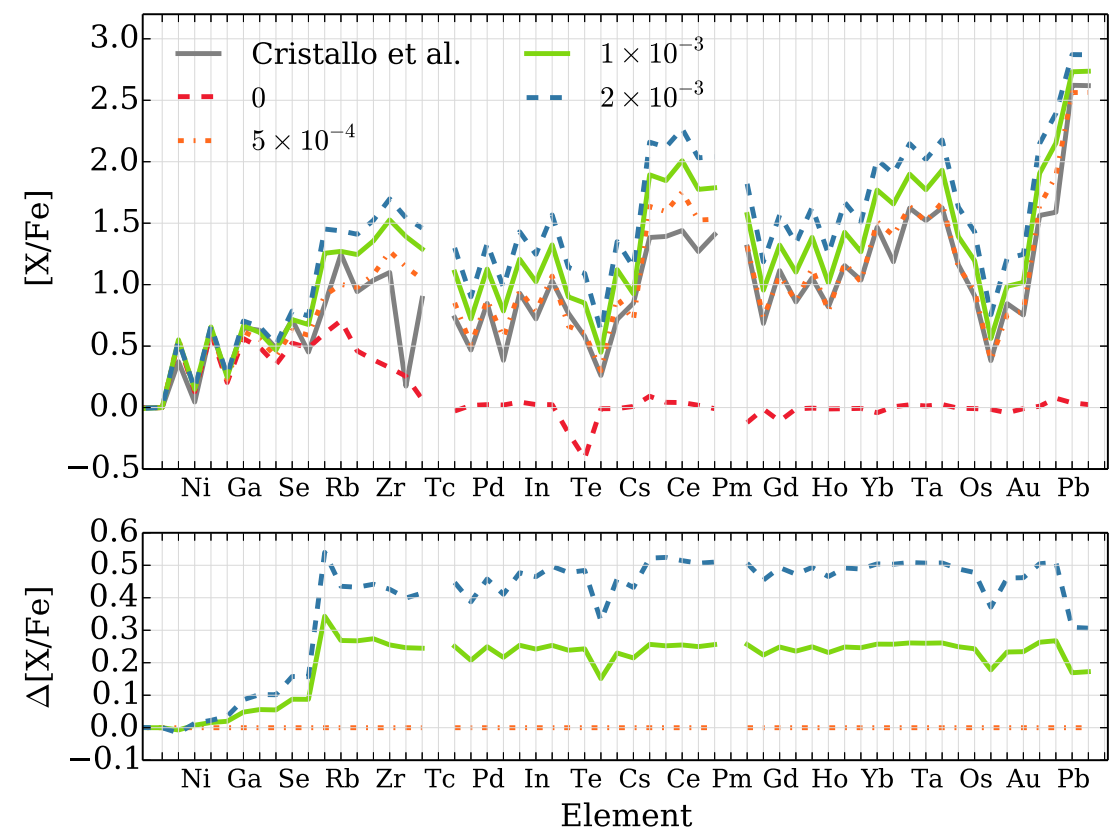

Figure 16. Final surface abundance ratios for each $3 M_{\odot}$ model with a different PMZ mass (in units of $M_{\odot}$ as indicated in the legend) for the elements heavier than Fe. The final surface abundances for the $3 M_{\odot}$ model of Cristallo et al. (2011) are also presented. The elements are ordered by increasing atomic number. For an explanation of why some abundances have a value less than solar, see the caption of Figure 4 and Section 2.

(A color version of this figure is available in the online journal.)

higher temperatures cause the ${ }^{13} \mathrm{C}$ pocket to form sooner and deeper in the intershell for the models with a more massive PMZ compared with the standard PMZ mass. The larger PMZ also results in larger ${ }^{13} \mathrm{C}$ and ${ }^{14} \mathrm{~N}$ pockets forming in the intershell. The extra ${ }^{14} \mathrm{~N}$ is captured by $\alpha$ particles during subsequent TPs to produce ${ }^{22} \mathrm{Ne}$. The increases in $\mathrm{Ne}$ and $\mathrm{Na}$ are therefore the result of the increased production of ${ }^{22} \mathrm{Ne}$, where the ${ }^{22} \mathrm{Ne}$ is dredged to the surface. Some of the newly synthesized ${ }^{22} \mathrm{Ne}$ is captured by protons in the $\mathrm{H}$-shell during the next interpulse period to make extra ${ }^{23} \mathrm{Na}$. When compared to the standard case, the model without a PMZ produces lower abundances with $[\mathrm{Ne} / \mathrm{Fe}]$ and $[\mathrm{Na} / \mathrm{Fe}]$ showing deficiencies of approximately -0.2 dex.

Of the elements between $\mathrm{Si}$ and $\mathrm{Mn}$, only $\mathrm{P}$ shows a nonnegligible production due to the increase in the mass of the $\mathrm{PMZ}$. There is only one stable isotope of $\mathrm{P}\left({ }^{31} \mathrm{P}\right)$ and it can be produced through neutron capture in AGB stars. The increase in $[\mathrm{P} / \mathrm{Fe}]$ with increasing $\mathrm{PMZ}$ mass is due to the increased number of neutrons available for neutron capture.

The effect of the PMZ mass on the final surface abundance distribution for the neutron-capture elements is illustrated in Figure 16. The height of the abundance peaks increases with increasing PMZ mass with the general shape of the distribution of the $l s$ and $h s$ elements remaining the same. The higher temperatures reached by a more massive PMZ increases the rate of the ${ }^{13} \mathrm{C}(\alpha, n){ }^{16} \mathrm{O}$ reaction resulting in a higher peak neutron density. This has the effect of increasing the efficiency of the branching points at ${ }^{85} \mathrm{Kr}$ and ${ }^{86} \mathrm{Rb}$ producing more ${ }^{86} \mathrm{Kr}$ and ${ }^{87} \mathrm{Rb}$, both of which have a magic number of neutrons. Figure 16 shows that $[\mathrm{Kr} / \mathrm{Fe}]$ has the largest increase between the model with a PMZ mass of $2 \times 10^{-3} M_{\odot}$ and the model with the standard PMZ.

We attribute the smaller increase in the $\mathrm{Pb}$ abundance with increasing PMZ mass (compared to the $l s$ and $h s$ elements) to the lower neutron exposure experienced in each ${ }^{13} \mathrm{C}$ pocket (see, e.g., Gallino et al. 1998). Compared to the model with the standard PMZ mass, the neutron exposures for models with a more massive PMZ become increasingly lower with each interpulse period. Another result of increased ${ }^{13} \mathrm{C}$ burning temperatures is that the ${ }^{13} \mathrm{C}$ nuclei are consumed faster and the duration of the ${ }^{13} \mathrm{C}$ pocket is shorter for the models with a more massive PMZ.

When a PMZ is not added in the post-processing nucleosynthesis calculations, the effect of the ${ }^{22} \mathrm{Ne}$ neutron source is more evident. In this case, the largest final abundance occurs for $[\mathrm{Rb} / \mathrm{Fe}]$ due to branching points opening in the $s$-process path at $\mathrm{Rb}$. The much lower neutron exposure however implies minimal production of second $s$-process peak elements and $\mathrm{Pb}$.

In contrast to our models with a PMZ, the model of Cristallo et al. (2011) has $\mathrm{Rb}$ as the most enhanced first $s$-process peak element. However, our $3 M_{\odot}$ model (with the standard PMZ mass) has a slightly higher $\mathrm{Rb}$ yield than the Cristallo et al. (2011) model, with a net yield of $2.41 \times 10^{-8} M_{\odot}$ compared to $1.81 \times 10^{-8} M_{\odot}$. This is due to the faster increase in Rb with TP number where $[\mathrm{Rb} / \mathrm{Fe}]$ asymptotically approaches 0.8 and more of this enriched material is then ejected through mass loss. The Cristallo et al. (2011) model has a lower abundance of second $s$-process peak elements but a higher $\mathrm{Pb}$ abundance than the model with the standard PMZ mass of $5 \times 10^{-4} M_{\odot}$.

All the models with a PMZ produce relatively high abundances of neutron-capture elements (Figure 17) and the values of $[l s / \mathrm{Fe}],[h s / \mathrm{Fe}]$, and $[\mathrm{Pb} / \mathrm{Fe}]$ increase with increasing PMZ mass. When comparing the model with a PMZ of $2 \times 10^{-3} M_{\odot}$ to the model with the standard PMZ, $[l s / \mathrm{Fe}]$ increases by $0.43 \mathrm{dex}$ while for $[h s / \mathrm{Fe}]$ the increase is $0.52 \mathrm{dex}$. For $[\mathrm{Pb} / \mathrm{Fe}]$ there is an increase of 0.3 dex.

Figure 17 also highlights the effect of changing the mass of the $\mathrm{PMZ}$ on the intrinsic $s$-process indicators $[h s / l s]$ and $[\mathrm{Pb} / h s]$. For the models with a PMZ, there is an absolute difference of only 0.08 for the $[h s / l s]$. The small change in $[h s / l s]$ is due to the abundances reaching equilibrium (see Lugaro et al. 2012). 

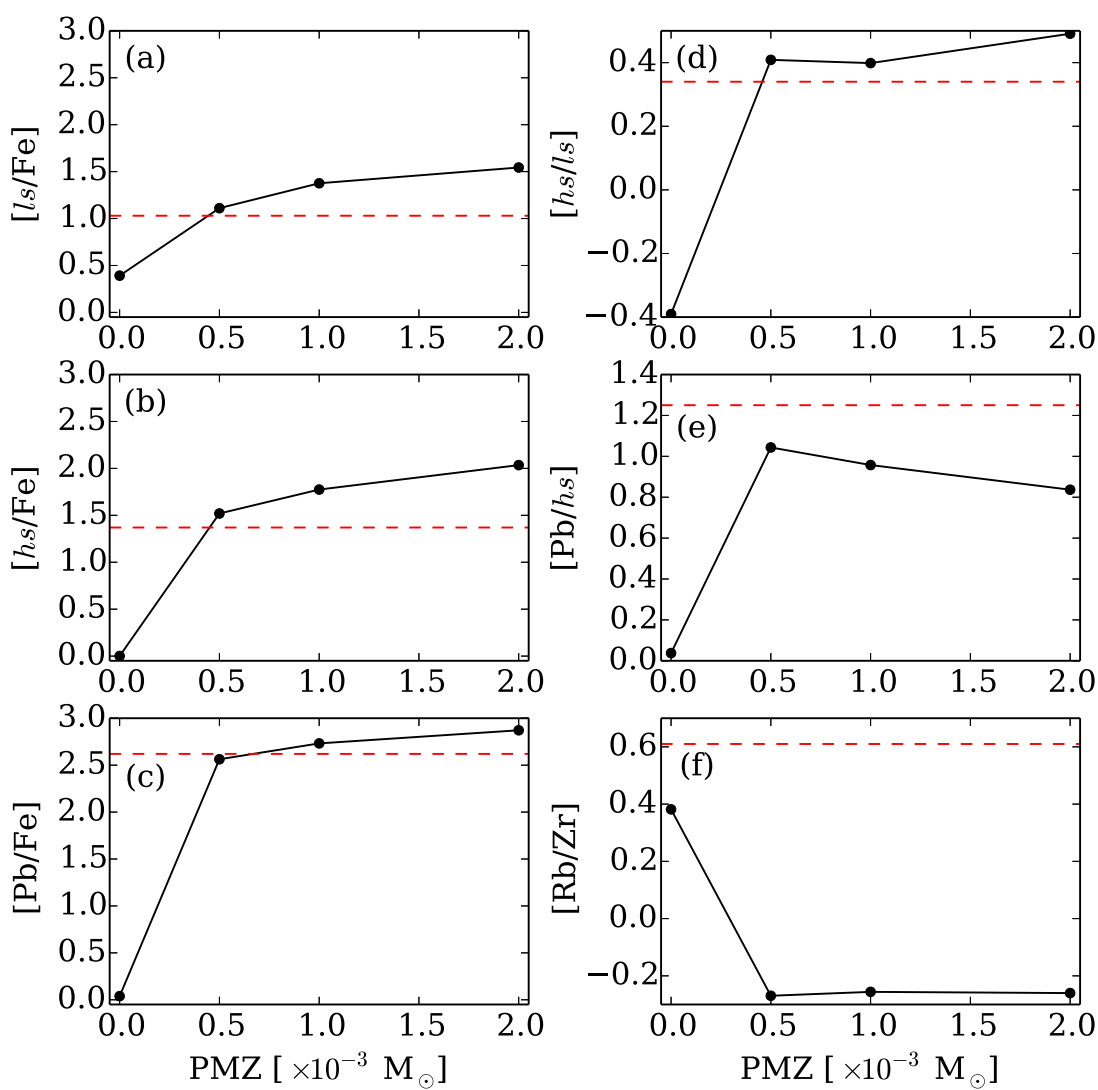

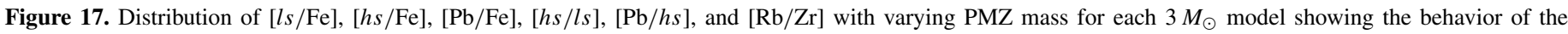
$s$-process peaks. The results from Cristallo et al. (2011) are shown as a horizontal dashed line.

(A color version of this figure is available in the online journal.)

For $[\mathrm{Pb} / h s]$ there is a decrease of 0.21 dex when increasing the PMZ mass from $5 \times 10^{-4}$ to $2 \times 10^{-3} M_{\odot}$. This is a result of the lower neutron exposure when the PMZ mass is higher.

Despite the different approaches, there is a reasonable agreement between the two groups, as testified by the $s$-process indicators reported in Figure 17. However, there is a disagreement between the final abundance of $[\mathrm{Rb} / \mathrm{Zr}]$ between the Cristallo et al. (2011) model and our models with a PMZ. The models presented here have a sub-solar $[\mathrm{Rb} / \mathrm{Zr}]$ ratio of approximately -0.25 , whereas the Cristallo et al. (2011) model has a ratio of $\sim 0.6$ due to the higher predicted $\mathrm{Rb}$ abundance. The model without a PMZ is the only model that shows a $[\mathrm{Rb} / \mathrm{Zr}]$ ratio above solar, which is a consequence of the ${ }^{22} \mathrm{Ne}(\alpha, n)^{25} \mathrm{Mg}$ reaction being the only source of neutrons.

Using our method for including a ${ }^{13} \mathrm{C}$ pocket, it is difficult to select an appropriate mass (and profile) for the $\mathrm{PMZ}$ in models in the transition phase between low- and intermediate mass. The models of Cristallo et al. $(2009,2011)$ use convective boundary mixing with an exponential decline in velocity to handle the discontinuity in the radiative gradient due to the abrupt change in opacities due to the TDU episodes. This leads to a deeper TDU and to protons being partially mixed into the core. The formation of a ${ }^{13} \mathrm{C}$ pocket then follows. Such a treatment of convective boundary mixing results in deeper TDU relative to our models. The mixing of protons inward in mass makes use of a free parameter $\beta$, with higher values of $\beta$ resulting in more efficient TDU. However, the effective mass of the ${ }^{13} \mathrm{C}$ pocket does not increase with increasing values of $\beta$. The mass of the ${ }^{13} \mathrm{C}$ pocket is at its largest when $\beta=0.1$. A lower or higher value of $\beta$ results in a lower abundance of neutron-capture elements.
Our PMZ, which is added during post-processing calculations, assumes a constant mass for the proton profile at each TDU episode. In contrast, the ${ }^{13} \mathrm{C}$ pockets of Cristallo et al. (2009, 2011) reduce in mass along the AGB, following the progressive shrinking in mass of the He-intershell.

\section{COMPARISON TO POST-AGB STARS}

We compare the final surface abundance predictions to three $s$ process rich post-AGB stars in the Large Magellanic Cloud (van Aarle et al. 2013; De Smedt et al. 2014): J050632.10-714229.8, J052043.86-692341.0, and J053250.69-713925.8. The postAGB stars have a metallicity of $[\mathrm{Fe} / \mathrm{H}] \approx-1.2$ and their initial masses are between 1 and $1.5 M_{\odot}$ (van Aarle et al. 2013). In Figure 18, we present the abundances determined by van Aarle et al. (2013) with upper limits of the $\mathrm{Pb}$ abundance from De Smedt et al. (2014) and the predicted final surface abundances from the models between 1 and $2 M_{\odot}$.

For J052043 and J053250, the $2 M_{\odot}$ model is the best match to the neutron-capture abundances of the $l s$ and $h s$ elements. These initial masses are higher than the $1-1.5 M_{\odot}$ estimated by van Aarle et al. (2013). The abundances of the $l s$ elements for $\mathrm{J} 050632$ also match the $2 M_{\odot}$ predictions however the $h s$ elements are better matched by the 1.25 or $1.5 M_{\odot}$ models.

As noted by De Smedt et al. (2014), the observed upper limits of the $\mathrm{Pb}$ abundance are well below the predicted values. This is in conflict with calculations of AGB models including those presented here. Model predictions of low-metallicity AGB stars suggest that the $\mathrm{Pb}$ abundance should be higher than that of the second $s$-process peak (Gallino et al. 1998). Piersanti 

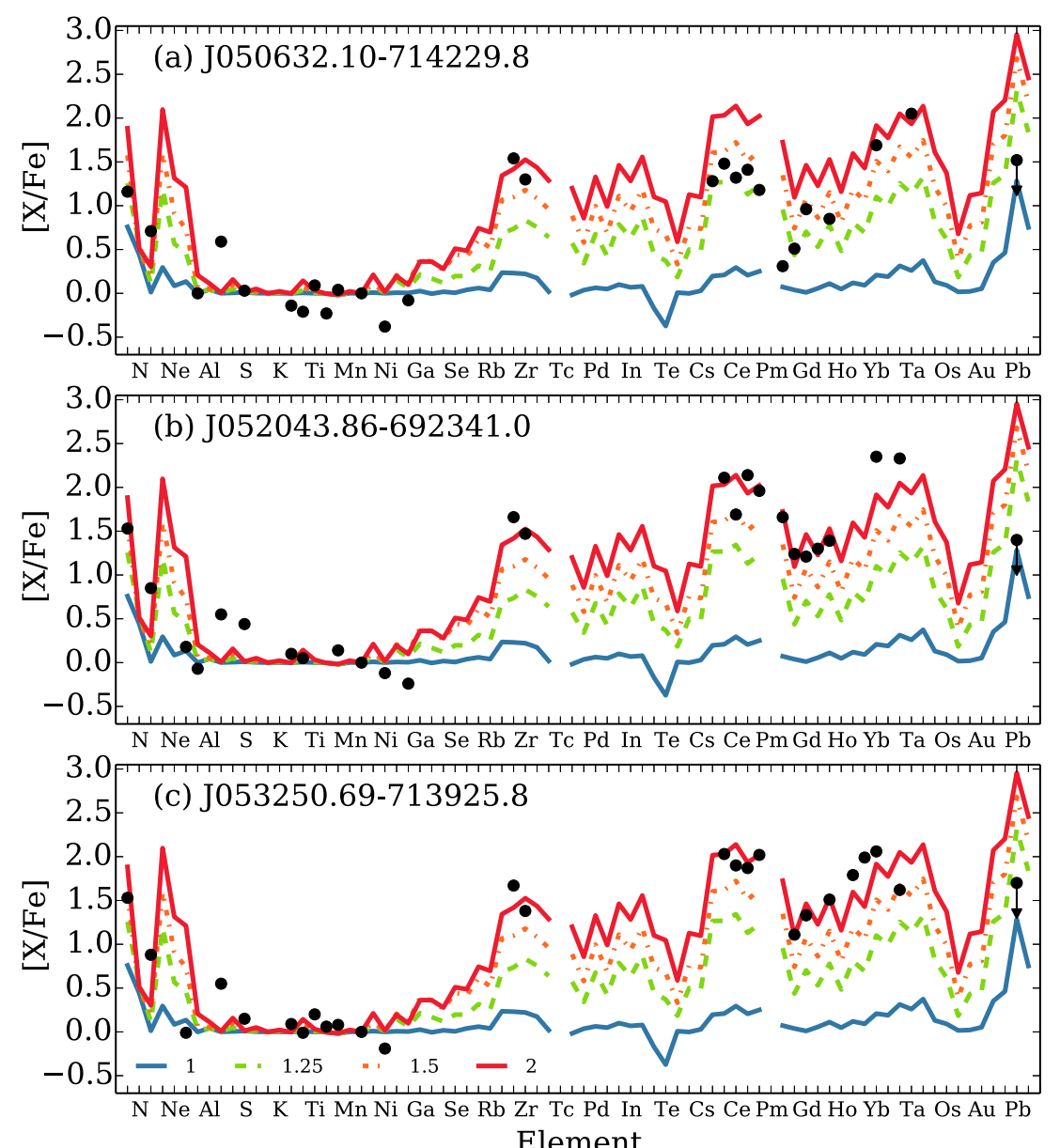

Figure 18. Comparison of three post-AGB stars (van Aarle et al. 2013; De Smedt et al. 2014) to four low-mass AGB models $\left(1,1.25,1.5\right.$, and $2 M_{\odot}$ ). The legend (in units of $M_{\odot}$ ) is shown in the bottom panel. The post-AGB abundances are shown as black points with the Pb abundance being only an upper limit.

(A color version of this figure is available in the online journal.)

et al. (2013) noted, using theoretical models of AGB stars, that rotation could decrease the final $[\mathrm{Pb} / \mathrm{Fe}]$ abundance, down to a value of 1.6 (from 2.8 for the model with no rotation) for a $1.5 M_{\odot}$ model with a rotation velocity of $120 \mathrm{~km} \mathrm{~s}^{-1}$ and $[\mathrm{Fe} / \mathrm{H}]$ of -1.7 . The presence of rotation also decreases the $[h s / l s]$ and $[\mathrm{Pb} / h s]$ ratios.

The observed values for $[\mathrm{C} / \mathrm{Fe}]$ are lower than the predictions of the best-matched model whereas $[\mathrm{O} / \mathrm{Fe}]$ is observed to be overabundant. One possibility for the high $[\mathrm{O} / \mathrm{Fe}]$ abundance is that the initial composition for the post-AGB stars was enhanced in $\mathrm{O}$ and $\mathrm{Si}$ compared to the scaled-solar initial composition used in the models. The required enhancements in the initial composition of $[\mathrm{O} / \mathrm{Fe}]$ to match the abundances of the post-AGB stars range from 0.41 to 0.58 . High abundances of other $\alpha$ elements $(\mathrm{Mg}, \mathrm{Ca}$, and $\mathrm{Ti})$ are not observed in the post-AGB stars.

\section{DISCUSSION OF UNCERTAINTIES}

There are many uncertainties in the input physics used for modeling AGB stars including convection, mass loss, extra mixing, reaction rates, rotation, and low-temperature opacities. It is therefore important to understand the role that these uncertainties have on the theoretical predictions. In this section we focus on a few uncertainties that can substantially affect the calculation of yields for neutron-capture elements; namely, mass loss, reaction rates, convection (and TDU), and the addition of $\mathrm{a}^{13} \mathrm{C}$ pocket.

The mass loss rate affects the lifetime of the AGB phase and the number of TDU episodes experienced. Therefore, mass loss plays a key role in influencing the chemical yields (see Stancliffe \& Jeffery 2007; Karakas 2010). With a more efficient mass loss rate, and hence a shorter AGB phase, lower yields of neutron-capture elements are expected as a smaller amount of enriched material is dredged to the surface to be expelled into the interstellar medium. Mass loss rates are difficult to determine from observations and require the modeling of dust properties and radiative transfer. Cristallo et al. (2009) compared models of $2 M_{\odot}$ with $Z=0.0001$ using two different mass-loss prescriptions: one with a Reimers $(1975, \eta=0.4)$ prescription, and the standard case which uses a prescription similar to Vassiliadis \& Wood (1993) but updated with more recent infrared observations (see Straniero et al. 2006). The model with the Reimers (1975) prescription shows an increase in the final surface abundance of the neutron-capture elements as a result of a longer AGB lifetime. However, it was found that the $s$-process indicators, $[h s / l s]$ and $[\mathrm{Pb} / h s]$, are less sensitive to the duration of the AGB phase as the largest ${ }^{13} \mathrm{C}$ pockets occur in the first few TPs and produce the largest increase in the $s$-process abundances. This sets the abundance ratio of the $s$-process indicators early in the AGB phase for the low-mass models. For the intermediate-mass models presented here, the 
values of the $s$-process indicators decrease over time and do not reach a constant ratio (see Figure 11).

The uncertainties in the reaction rates can also have an impact on the production of the neutron-capture elements. In particular for $s$-process nucleosynthesis, the reaction rates of the neutron sources, ${ }^{13} \mathrm{C}(\alpha, n){ }^{16} \mathrm{O}$ and ${ }^{22} \mathrm{Ne}(\alpha, n){ }^{25} \mathrm{Mg}$, can affect the number of neutrons produced per Fe seed. The ${ }^{13} \mathrm{C}(\alpha, n){ }^{16} \mathrm{O}$ reaction can only be measured experimentally at high energies and extrapolated to energies that occur during $s$-process nucleosynthesis. Guo et al. (2012) present an updated measurement for the reaction rate of ${ }^{13} \mathrm{C}(\alpha, n){ }^{16} \mathrm{O}$ and compares to previous measurements using AGB models with $s$-process nucleosynthesis. Relative to the reaction rates from Caughlan \& Fowler (1988) and Angulo et al. (1999), they find that if all the ${ }^{13} \mathrm{C}$ is destroyed in radiative conditions minimal variations of up to $5 \%$ occur for the neutron-capture elements. If some ${ }^{13} \mathrm{C}$ is destroyed inside a convective TP, the updated reaction rate has a larger effect on the abundance of the neutron-capture elements, with up to $25 \%$ variation for $\mathrm{Pb}$. The conditions where ${ }^{13} \mathrm{C}$ is burnt convectively occur in low-mass stars when there is incomplete radiative burning of ${ }^{13} \mathrm{C}$ during an interpulse or if there is proton ingestion in a TP. In the models presented here, we use the ${ }^{13} \mathrm{C}(\alpha, n){ }^{16} \mathrm{O}$ reaction rate taken from Heil et al. (2008) which is consistent with the updated measurement presented by Guo et al. (2012).

Concerning the uncertainties associated with convection and mixing length theory, a different $\alpha$ value will alter the amount of material mixed to the surface (see Boothroyd \& Sackmann 1988). Cristallo et al. (2009) investigated a $2 M_{\odot}$ model of $Z=0.0001$ with two different $\alpha$ values: 1.8 and 2.15 . The lower $\alpha$ value resulted in lower temperatures at the bottom of the pulse driven convective zone produced by a TP. Another consequence of the lower $\alpha$ value was less efficient TDU with $9.54 \times 10^{-2} M_{\odot}$ dredged up compared to $1.6 \times 10^{-1} M_{\odot}$. This decrease in $M_{\text {TDU }}$ occurs despite the standard model having one extra TP and results in lower final abundances. The $s$-process indicators for the low-mass models are less affected as they are more sensitive to the metallicity and to the mass of the ${ }^{13} \mathrm{C}$ pocket.

As mentioned in Section 1, low-mass models require extra mixing of protons to form the ${ }^{13} \mathrm{C}$ pocket (Karakas \& Lattanzio 2014). We have shown in Section 6 that changing the PMZ mass has an effect on the final surface abundances, particularly on the abundance of the neutron-capture elements. Shingles \& Karakas (2013) investigated varying the mass of the PMZ to match abundances in planetary nebulae and found that the predicted $\mathrm{Ne}$ abundance is sensitive to the size of the ${ }^{13} \mathrm{C}$ pocket. The lack of understanding of the mechanism responsible for the formation of the ${ }^{13} \mathrm{C}$ pocket highlights the uncertainties related to predicting yields of $s$-process nucleosynthesis. We refer the reader to Bisterzo et al. (2014) and Trippella et al. (2014) for further discussion on this point. It is important to note that TDU and the formation of the ${ }^{13} \mathrm{C}$ pocket should not be treated separately as is done with an added PMZ, particularly if the timescale for burning is shorter than the mixing timescale (Goriely \& Siess 2004).

The effect of rotation on the production of neutron-capture elements in AGB models has been studied by Herwig et al. (2003), Siess et al. (2004), and Piersanti et al. (2013). It was determined that rotation reduces the neutron flux as the ${ }^{13} \mathrm{C}$ pocket is mixed with the neutron poison ${ }^{14} \mathrm{~N}$. This reduction in the number of neutrons in turn may hinder the synthesis of the neutron-capture elements. The presence of rotation offers a possible solution to the lower than predicted $\mathrm{Pb}$ abundances in post-AGB stars. We do not consider rotation in our models.

\section{CONCLUSIONS}

We have presented new AGB stellar models for a range of initial masses from $1 M_{\odot}$ to $7 M_{\odot}$ for a metallicity of $Z=0.001([\mathrm{Fe} / \mathrm{H}]=-1.2)$ and a scaled-solar initial composition. In particular, $s$-process nucleosynthesis predictions for intermediate-mass AGB models of $Z=0.001$ are presented for the first time in the literature. We also present neutron-capture abundances and yields for a superAGB model of $7 M_{\odot}$ for the first time. Online tables are available presenting (for each stellar mass) evolutionary properties, final surface abundances (including $[\mathrm{X} / \mathrm{H}]$ and $[\mathrm{X} / \mathrm{Fe}]$ ) and yields for all elements, as well as isotope final surface abundances and yields for elements up to the Fe group.

We have presented in detail two representative AGB models, one low-mass model of $2 M_{\odot}$ and one intermediate-mass model of $5 M_{\odot}$. As a result of the activation of different neutron sources these models produce dissimilar abundance distributions. The low-mass models favor the production of $\mathrm{Pb}$ due to the ${ }^{13} \mathrm{C}(\alpha, n){ }^{16} \mathrm{O}$ reaction whereas the intermediate-mass models favor the production of $\mathrm{Rb}$ over other neutron-capture elements due to the activation of branching points by the ${ }^{22} \mathrm{Ne}(\alpha, n)^{25} \mathrm{Mg}$ reaction. The $[\mathrm{Rb} / \mathrm{Zr}]$ ratio, comparing two first $s$-process peak elements, is mass dependent with the intermediate-mass models showing an enhancement of $\sim 0.4 \mathrm{dex}$. The low-mass models show a sub-solar value down to -0.8 dex for the $2 M_{\odot}$ model.

The new predictions are compared to the $Z=0.001$ models of Cristallo et al. $(2009,2011)$ and Marigo et al. (2013), for masses in common between the various studies. The differences in the final surface abundances and yields between the calculations can be attributed to the choice of input physics such as the treatment of convective borders. The elemental yield predictions of the models presented here are comparable to those by Cristallo et al. (2011). The $s$-process indicators $[l s / \mathrm{Fe}]$ and $[h s / \mathrm{Fe}]$ agree to within $0.36 \mathrm{dex}$, with the largest difference occurring for the $1.5 M_{\odot}$ models for $[h s / \mathrm{Fe}]$. For $[\mathrm{Pb} / \mathrm{Fe}]$, the difference is less than 0.19 dex.

We also investigated the uncertainty in the addition of $a{ }^{13} \mathrm{C}$ pocket by varying the mass of the PMZ in the $3 M_{\odot}$ model. The $3 M_{\odot}$ model is in the transition zone between the lower mass models and the more massive models. Increases in the mass of the PMZ result in enhancements in the abundances of neutroncapture elements and a number of light elements $(\mathrm{O}, \mathrm{F}, \mathrm{Ne}$, $\mathrm{Na}, \mathrm{Mg}, \mathrm{Al}$, and $\mathrm{P})$. The intrinsic $s$-process indicator $[h s / l s]$ is shown to be weakly dependent on the mass of the PMZ, whereas $[\mathrm{Pb} / h s]$ decreases with increasing $\mathrm{PMZ}$ mass for the $3 M_{\odot}$ model due to lower neutron exposures.

One application of the AGB stellar models presented is a comparison of three low-metallicity post-AGB stars to the model predictions. Other applications include chemical evolution studies (e.g., Bisterzo et al. 2014) and the study of planetary nebulae in our Galaxy (e.g., Karakas \& Lugaro 2010) as well as external galaxies. The models presented here have been used in the interpretation of measured abundances of globular cluster stars (Yong et al. 2014a, 2014b), a chemically peculiar star in the Aquarius co-moving group (Casey et al. 2014), and the $s$-process component of M4 and M22 (Shingles et al. 2014).

We thank the referee whose thorough comments have improved the clarity of the paper. The authors are grateful for the 
support of the NCI National Facility at the ANU. C.K.F. is grateful for the financial support through an ANU PhD scholarship. A.I.K. and M.L. are supported through an Australian Research Council Future Fellowship (FT110100475 and FT100100305, respectively). This research has made use of NASA's Astrophysics Data System.

\section{REFERENCES}

Abia, C., Busso, M., Gallino, R., et al. 2001, ApJ, 559, 1117

Abia, C., Cunha, K., Cristallo, S., et al. 2010, ApJL, 715, L94

Abia, C., Recio-Blanco, A., de Laverny, P., et al. 2009, ApJ, 694, 971

Angulo, C., Arnould, M., Rayet, M., et al. 1999, NuPhA, 656, 3

Arnould, M., Goriely, S., \& Jorissen, A. 1999, A\&A, 347, 572

Asplund, M., Grevesse, N., Sauval, A. J., \& Scott, P. 2009, ARA\&A, 47,481

Bisterzo, S., Travaglio, C., Gallino, R., Wiescher, M., \& Käppeler, F. 2014, ApJ, 787, 10

Blöcker, T. 2001, ApS\&S, 275, 1

Böhm-Vitense, E. 1958, ZA, 46, 108

Boothroyd, A. I., \& Sackmann, I.-J. 1988, ApJ, 328, 671

Busso, M., Gallino, R., Lambert, D. L., Travaglio, C., \& Smith, V. V. 2001, ApJ, 557,802

Busso, M., Gallino, R., \& Wasserburg, G. J. 1999, ARA\&A, 37, 239

Casey, A. R., Keller, S., Alves-Brito, A., et al. 2014, MNRAS, 443, 828

Caughlan, G. R., \& Fowler, W. A. 1988, ADNDT, 40, 283

Cristallo, S., Piersanti, L., Straniero, O., et al. 2011, ApJS, 197, 17

Cristallo, S., Straniero, O., Gallino, R., et al. 2009, ApJ, 696, 797

De Smedt, K., Van Winckel, H., Kamath, D., et al. 2014, A\&A, 563, L5

Dinerstein, H. L., \& Geballe, T. R. 2001, ApJ, 562, 515

Doherty, C. L., Gil-Pons, P., Lau, H. H. B., Lattanzio, J. C., \& Siess, L. 2014a, MNRAS, 437, 195

Doherty, C. L., Gil-Pons, P., Lau, H. H. B., et al. 2014b, MNRAS, 441, 582

Fishlock, C. K., Karakas, A. I., \& Stancliffe, R. J. 2014, MNRAS, 438, 1741

Frost, C. A., Cannon, R. C., Lattanzio, J. C., Wood, P. R., \& Forestini, M. 1998, A\&A, 332, L17

Frost, C. A., \& Lattanzio, J. C. 1996, ApJ, 473, 383

Gallino, R., Arlandini, C., Busso, M., et al. 1998, ApJ, 497, 388

García-Hernández, D. A., Zamora, O., Yagüe, A., et al. 2013, A\&A, 555, L3

Goriely, S., \& Siess, L. 2004, A\&A, 421, L25

Guo, B., Li, Z. H., Lugaro, M., et al. 2012, ApJ, 756, 193

Heil, M., Detwiler, R., Azuma, R. E., et al. 2008, PhRvC, 78, 25803

Herwig, F. 2004, ApJS, 155, 651

Herwig, F. 2005, ARA\&A, 43, 435

Herwig, F., Langer, N., \& Lugaro, M. 2003, ApJ, 593, 1056

Iglesias, C. A., \& Rogers, F. J. 1996, ApJ, 464, 943

Iliadis, C., Longland, R., Champagne, A. E., Coc, A., \& Fitzgerald, R. 2010, NuPhA, 841, 31

Izzard, R. G., Glebbeek, E., Stancliffe, R. J., \& Pols, O. R. 2009, A\&A, 508,1359
Izzard, R. G., Tout, C. A., Karakas, A. I., \& Pols, O. R. 2004, MNRAS, 350,407

Jaeger, M., Kunz, R., Mayer, A., et al. 2001, PhRvL, 87, 202501

Johnson, J. A., Herwig, F., Beers, T. C., \& Christlieb, N. 2007, ApJ, 658, 1203

Jorissen, A., Smith, V. V., \& Lambert, D. L. 1992, A\&A, 261, 164

Kamath, D., Karakas, A. I., \& Wood, P. R. 2012, ApJ, 746, 20

Karakas, A., \& Lattanzio, J. C. 2007, PASA, 24, 103

Karakas, A. I. 2010, MNRAS, 403, 1413

Karakas, A. I., Campbell, S. W., \& Stancliffe, R. J. 2010, ApJ, 713, 374

Karakas, A. I., \& Lattanzio, J. C. 2014, PASA, 31, 30

Karakas, A. I., \& Lugaro, M. 2010, PASA, 27, 227

Kobayashi, C., Karakas, A. I., \& Umeda, H. 2011, MNRAS, 414, 3231

Lattanzio, J. C. 1986, ApJ, 311, 708

Lau, H. H. B., Gil-Pons, P., Doherty, C., \& Lattanzio, J. 2012, A\&A, 542, 1

Letarte, B., Hill, V., Tolstoy, E., et al. 2010, A\&A, 523, 17

Lodders, K. 2003, ApJ, 591, 1220

Lugaro, M., Karakas, A. I., Stancliffe, R. J., \& Rijs, C. 2012, ApJ, 747, 2

Lugaro, M., Tagliente, G., Karakas, A. I., et al. 2014, ApJ, 780, 95

Lugaro, M., Ugalde, C., Karakas, A. I., et al. 2004, ApJ, 615, 934

Marigo, P., \& Aringer, B. 2009, A\&A, 508, 1539

Marigo, P., Bressan, A., Nanni, A., Girardi, L., \& Pumo, M. L. 2013, MNRAS, 434, 488

Marino, A. F., Sneden, C., Kraft, R. P., et al. 2011, A\&A, 532, 8

Meléndez, J., \& Cohen, J. G. 2007, ApJL, 659, L25

Meléndez, J., \& Cohen, J. G. 2009, ApJ, 699, 2017

Mowlavi, N., Jorissen, A., \& Arnould, M. 1996, A\&A, 311, 803

Piersanti, L., Cristallo, S., \& Straniero, O. 2013, ApJ, 774, 98

Recio-Blanco, A., de Laverny, P., Worley, C., et al. 2012, A\&A, 538, 117

Reimers, D. 1975, Mem. Soc. Roy. Sci. Liege, 8, 369

Romano, D., Karakas, A. I., Tosi, M., \& Matteucci, F. 2010, A\&A, 522, 32

Shingles, L. J., \& Karakas, A. I. 2013, MNRAS, 431, 2861

Shingles, L. J., Karakas, A. I, Hirschi, R., et al. 2014, ApJ, 795, 34

Siess, L. 2007, A\&A, 476, 893

Siess, L., Goriely, S., \& Langer, N. 2004, A\&A, 415, 1089

Smith, C. L., Zijlstra, A. A., \& Dinerstein, H. L. 2014, MNRAS, 441, 3161

Stancliffe, R. J., \& Jeffery, C. S. 2007, MNRAS, 375, 1280

Straniero, O., Cristallo, S., \& Gallino, R. 2009, PASA, 26, 133

Straniero, O., Cristallo, S., \& Piersanti, L. 2014, ApJ, 785, 77

Straniero, O., Gallino, R., Busso, M., et al. 1995, ApJL, 440, L85

Straniero, O., Gallino, R., \& Cristallo, S. 2006, NuPhA, 777, 311

Trippella, O., Busso, M., Maiorca, E., Käppeler, F., \& Palmerini, S. 2014, ApJ, 787,41

van Aarle, E., Van Winckel, H., De Smedt, K., Kamath, D., \& Wood, P. R. 2013, A\&A, 554, 106

van Raai, M. A., Lugaro, M., Karakas, A. I., Garcia-Hernandez, D. A., \& Yong, D. 2012, A\&A, 540, 44

Vassiliadis, E., \& Wood, P. R. 1993, ApJ, 413, 641

Ventura, P., \& D'Antona, F. 2008, A\&A, 479, 805

Ventura, P., \& D'Antona, F. 2010, MNRAS, 402, L72

Ventura, P., \& Marigo, P. 2010, MNRAS, 408, 2476

Yong, D., Alves-Brito, A., Da Costa, G. S., et al. 2014a, MNRAS, 439, 2638

Yong, D., Roederer, I. U., Grundahl, F., et al. 2014b, MNRAS, 441, 3396 